\title{
Application of Energy-Based Power System Features for Dynamic Security Assessment
}

\author{
by \\ Janath Chaminda Geeganage
}

A Thesis submitted to the Faculty of Graduate Studies of The University of Manitoba in partial fulfilment of the requirements of the degree of DOCTOR OF PHILOSOPHY

The Department of Electrical and Computer Engineering The University of Manitoba Winnipeg, Manitoba, Canada 


\section{Abstract}

To date, the potential of on-line Dynamic Security Assessment (DSA) - to monitor, alert, and enhance system security - is constrained by the longer computational cycle time. Traditional techniques requiring extensive numerical computations makes it challenging to complete the assessment within an acceptable time. Longer computational cycles produce obsolete security assessment results as the system operating point evolves continuously. This thesis presents a DSA algorithm, based on Transient Energy Function (TEF) method and machine learning, to enable frequent computational cycles in on-line DSA of power systems.

The use of selected terms of the TEF as pre-processed input features for machine learning demonstrated the ability to successfully train a contingency-independent classifier that is capable of classifying stable and unstable operating points. The network is trained for current system topology and loading conditions. The classifier can be trained using a small dataset when the TEF terms are used as input features. The prediction accuracy of the proposed scheme was tested under the balanced and unbalanced faults with the presence of voltage sensitive and dynamic loads for different operating points. The test results demonstrate the potential of using the proposed technique for power system on-line DSA. Power system devices such as HVDC and FACTS can be included in the algorithm by incorporating the effective terms of a corresponding TEF.

An on-line DSA system requires the integration of several functional components. Some of these components already exist and the others are newly introduced to the 
power system. The practicality of the proposed technique in terms of a) critical data communications aspects b) computational hardware requirements; and c) capabilities and limitations of the tools in use was tested using an implementation of an on-line DSA system. The test power system model was simulated using a real-time digital simulator. The other functional units were distributed over the Local Area Network (LAN). The implementation indicated that an acceptable computational cycle time can be achieved using the proposed method.

In addition, the work carried out during this thesis has produced two tools that can be used for $a$ ) web-based automated data generation for power system studies; and $b$ ) testing of on-line DSA algorithms. 


\section{Acknowledgements}

First, my sincere gratitude to Professor Udaya Annakkage for providing me the invaluable opportunity to work with him and for mentoring me with continuous guidance, encouragement and appreciation. I would also like to thank the advisory committee members for their comments and feedback towards the enhancement of this thesis.

I would like to appreciate the funding and infrastructure support provided to me by Manitoba Hydro, NSERC Canada, MITACS Accelerate Canada, University of Manitoba and RTDS Technologies.

The support given to me by the academic, technical and administrative staff and the fellow students of Power Systems group is highly appreciated. Further, I am thankful to the staff of the System Performance and Systems Planning departments of Manitoba Hydro for facilitating me for several internships and for their valuable feedback.

I am also thankful to Professor H Sriyanada and to the Open University of Sri Lanka for providing me the opportunity for engineering studies.

I express gratitude to my wife and two kids for their understanding throughout this period. Finally, I extend gratitude to my parents, parents-in-law, brother, sister and brother-in-law for all their support during my studies.

Janath Geeganage

April 2016 


\section{Dedication}

To my parents -Kamala and Karunasena. 


\section{Contents}

Abstract ............................. i

Acknowledgements ........................... ii

Dedication . . . . . . . . . . . . . . . . iv

List of Tables . . . . . . . . . . . . . . . . . ix

List of Figures . . . . . . . . . . . . . . . . . . $x$

Acronyms $\quad$ xii

List of Symbols $\quad$ XV

1 Introduction $\quad 1$

1.1 Real-time DSA . . . . . . . . . . . . . . . . 2

1.2 Classification of power system stability and security . . . . . . . . 3

1.3 Motivation . . . . . . . . . . . . . . 5

1.4 Research Objectives . . . . . . . . . . . . . . . . . . . . . 8

1.5 Thesis Overview . . . . . . . . . . . . . . . . 10

2 Power System Dynamic Security Assessment 11

2.1 Introduction . . . . . . . . . . . . . . . . . . 11

2.2 Techniques for fast DSA . . . . . . . . . . . . . . . . 11

2.2.1 Machine Learning Techniques . . . . . . . . . . . . . . . 11

2.2.2 Direct Assessment Methods . . . . . . . . . . . . . . . . . . . 15

2.2.3 Functional Representation of Stability Boundary . . . . . . . . 18

2.2.4 Using Hyperplanes and Nomograms . . . . . . . . . . . . . . . 19

2.2.5 Variable Time-step Integration and Early Termination Methods 21

2.2.6 Dynamic Equivalencing Techniques . . . . . . . . . . . . . 21

2.2.7 High Performance Computing . . . . . . . . . . . . . . 23

2.2.8 Other Approaches . . . . . . . . . . . . . . . . . . . 23

2.3 Modelling of Power System Components for DSA . . . . . . . . . . 24

2.3.1 Synchronous Generator Models . . . . . . . . . . . . . 25

2.3.2 Transmission Line Model . . . . . . . . . . . . . . . . . . . . . 28

2.3.3 Load Models . . . . . . . . . . . . . . . . . . . 28

2.3.4 Interfacing Components . . . . . . . . . . . . . 29

2.4 Concluding Remarks . . . . . . . . . . . . . . . . . . . 30 
3 Direct Methods for Power System Stability Assessment 31

3.1 Introduction . . . . . . . . . . . . . . . . . . . 31

3.1.1 Introduction to Direct Methods of Stability Assessment . . . 31

3.1.2 Stability in the Sense of Lyapunov _ . . . . . . . . . . . 32

3.1 .3 Lyapunov Function . . . . . . . . . . . . . . . . . . . . . . 33

3.1.4 Local and Global Stability Using the Lyapunov Theorem . . . 34

3.1.5 Stability Region of an Stable Equilibrium Point (SEP) . . . . 35

3.2 Application of Direct Methods to Power Systems . . . . . . . . . . 36

3.2 .1 TEF . . . . . . . . . . . . . . . . . 37

3.3 Numerical Energy Functions . . . . . . . . . . . . . . . . . . . . 41

3.4 Stability Assessment . . . . . . . . . . . . . . . . . . . . 41

3.4.1 Steps for Stability Assessment Using the Direct Method . . . . 41

3.4.2 Calculation of TEF Terms . . . . . . . . . . . . . . . 43

3.5 Methods for Critical Energy Calculation _. . . . . . . . . . 45

3.5.1 Potential Energy Boundary Surface (PEBS) Method . . . . . 46

3.5.2 Controlling Unstable Equilibrium Point (CUEP) Method . . . 47

3.5.3 Closest Unstable Equilibrium Point (UEP) Method . . . . . . 50

3.5.4 Boundary of Stability Region based Controlling Unstable Equilibrium Point (BCU) Method . . . . . . . . . . . . . 50

3.5 .5 Other Attempts . . . . . . . . . . . . . . . . 52

3.6 Limitations and Issues of Direct Method of Stability Assessment . . . 53

3.7 Concluding Remarks . . . . . . . . . . . . . . . . . 54

4 Automated Database Generation for Dynamic Security Assessment 55

4.1 Introduction . . . . . . . . . . . . . . . 55

4.2 Requirement Specifications . . . . . . . . . . . . . . 56

4.3 System Structure . . . . . . . . . . . . . . . . . . . . 57

4.3 .1 Inputs . . . . . . . . . . . . . . . . . . 58

4.4 Steady State Operating Point Generation . . . . . . . . . . . . . 59

4.5 System Specific Limit Checks and Corrective Actions . . . . . . . . 62

4.6 Automated Dynamic Simulations . . . . . . . . . . . . . . . 63

4.6 .1 Stability Classification . . . . . . . . . . . . . . . . 64

4.6.2 TEF Terms Computation . . . . . . . . . . . . . 64

4.7 Testing Process $\ldots \ldots \ldots \ldots$

4.8 Additional Features of the Software . . . . . . . . . . . . 67

4.8 .1 Web Interface . . . . . . . . . . . . . . . . . . 67

4.8 .2 Configurability . . . . . . . . . . . . . . . 67

4.8.3 Relational DBMS Integration and Study Reports . . . . . . 67

4.9 Concluding Remarks . . . . . . . . . . . . . . . . . . . . . 68

5 Proposed Method for Transient Stability Assessment (TSA) using Energy Based Power System Features and Machine Learning 69

5.1 Introduction . . . . . . . . . . . . . . . . . . . . . . . . 69

5.2 The Concept of Combining Direct Methods and Machine Learning . . 70

5.3 Proposed Method . . . . . . . . . . . . . . . . . 71 
5.3 .1 A Simple Example . . . . . . . . . . . . . . . . . . 71

5.3.2 Candidate Input Features of the TEF . . . . . . . . . . . 73

5.3.3 Application of Support Vector Machines (SVMs) for Classification 74

5.3.4 Two Simultaneous Processes: Training and Assessment . . . . 75

5.4 Simulation and Results . . . . . . . . . . . . . . . . . . . . 79

5.4 .1 Test System Model . . . . . . . . . . . . . . . . . . . . 79

5.4 .2 Data Generation . . . . . . . . . . . . . . . . 80

5.4 .3 Accuracy Indexes . . . . . . . . . . . . . . . . . . . . . 82

5.4.4 Feature Selection for Machine Learning . . . . . . . . . . . . 83

5.4.5 Accuracy of Classification for Smaller Training Datasets . . . 84

5.4.6 Classification Accuracy for Unbalanced Faults with the Presence of Voltage Sensitive and Dynamic Loads . . . . . . . . 85

5.4.7 Computational Performance and Resource Requirement . . . . 86

5.4.8 Inclusion of Flexible Alternative Current Transmission System (FACTS) Devices Including High Voltage Direct Current (HVDC)

Lines . . . . . . . . . . . . . . . . . . 87

5.4.9 Significance of the Proposed Method . . . . . . . . . . . 88

5.5 Concluding Remarks . . . . . . . . . . . . . . . . . . . . . . 89

6 Implementation of on-line DSA $\quad 90$

6.1 Introduction . . . . . . . . . . . . . . . . . 90

6.2 The Proposed Framework _. . . . . . . . . . . . . . . . . . . 91

6.2 .1 Requirement Specification _. . . . . . . . . . . . . . . 91

6.3 Overall Structure of the Proposed Framework . . . . . . . . . . . . 93

6.4 Real-Time Simulation of Power System Operation . . . . . . . . . . 95

6.5 Construction of the System Snapshot . . . . . . . . . . . . 97

6.6 Process A: Producing a Learned Network . . . . . . . . . . . . . . 101

6.6.1 Scalability of Process A . . . . . . . . . . . . . . . . 101

6.7 Process B: Security Assessment Using the Learned Network . . . . 102

6.8 Concurrent Implementation of Process A and Process B . . . . . . . 102

6.9 Continuation at a Network Topology Change . . . . . . . . . . . . 103

6.10 Other features . . . . . . . . . . . . . . . . . . . . . . . . . . 104

6.10 .1 Database Management System (DBMS) . . . . . . . . 104

6.10 .2 User Authentication . . . . . . . . . . . . . . . . . . . 104

6.10 .3 Web-based Interface . . . . . . . . . . . . . . . . . 104

6.11 Testing . . . . . . . . . . . . . . . . . . . . . . . . . 104

6.11 .1 Further Developments . . . . . . . . . . . . . . 105

6.12 Concluding Remarks . . . . . . . . . . . . . . . . 106

7 Conclusions, Contributions and Suggestions for Future Work 108

7.1 Conclusions . . . . . . . . . . . . . . . . . . . . . . . . . 108

7.2 Suggestions for Future Work . . . . . . . . . . . . . . . . . 111

Appendix A Derivation of the TEF for 3-Bus Test Power System 113 
Appendix B Energy Function for a Lossless SMIB System and the Equal Area Criterion (EAC)

Appendix C Test System Data: IEEE 10 Generator 39 Bus System 120

$\begin{array}{lr}\text { Appendix D Classification using SVM } & 126\end{array}$

D.1 Introduction . . . . . . . . . . . . . . . . . . 126

D.2 Support Vector Machines . . . . . . . . . . . . . . . . 126

D.3 Hard Margin SVM . . . . . . . . . . . . . . . . . . 127

D.3.1 Non-linear Transformation to Higher Dimensions . . . . . . . 128

D.3.2 Use of Kernels . . . . . . . . . . . . . . . . . . . . . . . . . . . . 129

D.3.3 Radial Basis Function (RBF) Kernel . . . . . . . . . . . . . 129

D.4 Soft Margin SVM . . . . . . . . . . . . . . . . . 130

D.4.1 Accounting for Margin Violations . . . . . . . . . . . 130

References 


\section{List of Tables}

5.1 Cross validated classification accuracy of two networks trained using raw and pre-processed inputs . . . . . . . . . . . . . . 72

5.25 -fold cross validation accuracy of two feature sets based on the four TEF terms . . . . . . . . . . . . . . . . . .

5.3 Training and validation accuracy of two networks trained without the numerically intensive PE3 term . . . . . . . . . . . . . .

5.4 Training and validation accuracy once the generator bus voltage related input features are incorporated . . . . . . . . . . . . . 84

5.5 Training and validation accuracy of networks trained using a half and a quarter sized training dataset . . . . . . . . . . . . .

5.6 Training and validation accuracy of networks trained using a dataset of 250 items for line to ground faults with the presence of voltage dependant and dynamic loads . . . . . . . . . . . . . . .

5.7 Training and validation accuracy of networks trained using a dataset of 125 items for line to ground faults with the presence of voltage dependant and dynamic loads . . . . . . . . . . . . . . .

C.1 Real and reactive power at load buses of the IEEE 10 generator 39 bus power system . . . . . . . . . . . . . . . . . 120

C.1 Real and reactive power at load buses of the IEEE 10 generator 39 bus power system . . . . . . . . . . . . . . . . . 121

C.2 Transmission Line Data of the IEEE 10 generator 39 bus power system. The quantities are specified in per unit on 100MVA . . . . . . . . . . 121

C.2 Transmission Line Data of the IEEE 10 generator 39 bus power system. The quantities are specified in per unit on 100MVA . . . . . . . . . . 122

C.3 Generator dynamic parameters - Salient Pole Generator Model . . . . 123

C.4 Exciter parameters - IEEE Type 1 Excitation System . . . . . . . . . 124

C.5 Power System Stabilizer (PSS) parameters - Speed sensitive stabilizing

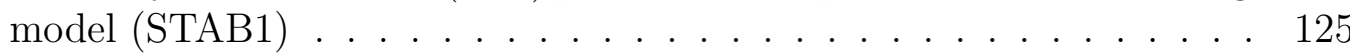




\section{List of Figures}

2-1 Kinetic energy variations of the Second Kick method . . . . . . . . 16

2-2 Conceptual view of an approximated security region . . . . . . . . 19

3-1 Concepts of stability (1: asymptotically stable, 2: marginally stable, 3: unstable, 4 : globally stable) . . . . . . . . . . . . . .

3-2 Drift of trajectories away from the SEP when the radial unboundedness not assured . . . . . . . . . . . . . . .

3-3 Evolution of trajectories within the stability boundary and on the sta-

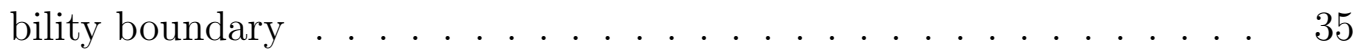

3-4 Total and potential energy variation during a fault-on trajectory computed using the TEF . . . . . . . . . . . . . . . 42

3-5 Contributions of the individual terms of the TEF for the potential energy 43

3-6 PEBS Crossing is identified at the zero crossing point of $f^{T}\left(\theta-\theta^{S}\right) . \quad 47$

3-7 Phase plot of the CUEP computation using the time domain method 49

3-8 Potential energy vs rotor angle, including two UEPs and the SEP for a Single Machine to Infinite Bus (SMIB) system . . . . . . . . . 51

4-1 Overall block diagram of Machine Learning for Dynamic Security Assessment (ML4DSA) . . . . . . . . . . . . . . . . 58

4-2 Overall block diagram of acceptable steady state operating point generation process. . . . . . . . . . . . . . . . 61

4-3 A screen-shot of the ML4DSA interface for acceptable operating point generation. . . . . . . . . . . . . . . . 62

4-4 Structure of a typical corrective actions folder compatible for ML4DSA. 63

4-5 Process flow of iterative dynamic simulation. . . . . . . . . . 65

4-6 Screen-shot of stability assessment of a dynamic simulation. . . . . . 66

5-1 Two simultaneous processes 1) data generation and training a network for the next period of time 2) security assessment using the previously trained network . . . . . . . . . . . . .

5-2 Process A: Flow chart for data generation and network training for the next computational cycle. . . . . . . . . . . . . . . 78

5-3 Single line diagram of the New England 39 bus 10 generator test power

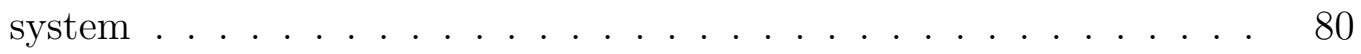

6-1 Overall block diagram of the real-time DSA framework . . . . . . . 94 
6-2 Boundaries of the two subsystems implemented on two Real Time Digital Simulator ${ }^{\circledR}$ (RTDS) racks (IEEE 39-bus 10-generator test power system $) \ldots \ldots \ldots \ldots \ldots \ldots \ldots \ldots$

6-3 Representation of the load curve in $15 \mathrm{~s}$ intervals for a single load . . 97

6-4 The format of a meter capture record in snapshot.csv and the 7 types of faciltated quantities . . . . . . . . . . . . . . 99

6-5 Representation of the load curve in $15 \mathrm{~s}$ intervals for a single load . . 99

6-6 Illustration of the real-time implementation of DSA . . . . . . . . 103

A-1 A diagram of the reduced 3-machine 9-bus test power system model . 113

B-1 SMIB system representation using EAC . . . . . . . . . . . 117

D-1 Possible solutions from different machine learning techniques . . . . 127

D-2 Margins of different solutions . . . . . . . . . . . . . 127

D-3 Feature space . . . . . . . . . . . . . . . . . . . . . . . . . . . . 129

D-4 Margin violation in a linearly separable dataset $\ldots \ldots \ldots \ldots . . \ldots 131$ 


\section{Acronyms}

AGC Automatic Generation Control.

ANN Artificial Neural Network.

API Application Programming Interface.

BCU Boundary of Stability Region based Controlling Unstable Equilibrium Point.

CLF candidate Lyapunov function.

COI Center of Inertia.

CUEP Controlling Unstable Equilibrium Point.

DAE Differential and Algebraic Equation.

DBMS Database Management System.

DSA Dynamic Security Assessment.

EAC Equal Area Criterion.

EEAC Extended Equal Area Criterion.

ELM Extreme Learning Machine.

EMS Energy Management System.

EMT Electro Magnetic Transient. 
FA False Alarm.

FACTS Flexible Alternative Current Transmission System.

FC False Classification.

FD False Dismissal.

GBH Global Bus Hub.

HVDC High Voltage Direct Current.

IRC Inter Rack Communication.

LAN Local Area Network.

LFE Low-Frequency Equivalent.

ML4DSA Machine Learning for Dynamic Security Assessment.

MRO Midwest Reliability Organization.

MVC Model View Controller.

NAS Network Attached Storage.

PEBS Potential Energy Boundary Surface.

PMU Phasor Measurement Unit.

PSS Power System Stabilizer.

PSSE Power System Simulator for Engineering ${ }^{\circledR}$.

RBF Radial Basis Function.

RTDS Real Time Digital Simulator ${ }^{\circledR}$. 
SCADA Supervisory control and data acquisition.

SEP Stable Equilibrium Point.

SMIB Single Machine to Infinite Bus.

SSA Static Security Assessment.

SSSC static series synchronous compensator.

SVM Support Vector Machine.

TEF Transient Energy Function.

TEM Transient Energy Margin.

TSA Transient Stability Assessment.

TSAT Transient Security Analysis Tool ${ }^{\circledR}$.

UEP Unstable Equilibrium Point.

UPFC unified power flow controller.

WAMS Wide Area Measurement System.

Web2Py Web2Py ${ }^{\mathrm{TM}}$ Web Framework.

WECC Western Electricity Coordinating Council. 


\section{List of Symbols}

$C$ Cost parameter.

$B$ Susceptance (pu).

$\delta_{0}$ Rotor angle of the COI (rad).

$\delta$ Generator rotor angle (rad).

$D$ Damping constant (pu).

$E$ Bus voltage magnitude (pu).

$G$ Conductance (pu).

$H$ Inertia constant (pu).

$K$ Kernel function.

$\mathcal{L}$ Lagrangian equation.

$\omega_{0}$ Angular speed of the COI ( $\left.\mathrm{rad} / \mathrm{sec}\right)$.

$\omega_{s}$ Synchronous angular speed of the rotor $(\mathrm{rad} / \mathrm{sec})$.

$\tilde{\omega}$ Angular speed of a generator relative to the COI ( $\mathrm{rad} / \mathrm{s})$.

$\omega$ Angular speed of a generator $(\mathrm{rad} / \mathrm{sec})$. 
$P_{e}^{F}$ Maximum real power absorbed by the fault-on network (pu).

$P_{e}^{\text {Max }}$ Maximum real power absorbed by the postfault network (pu).

$P_{e}$ Electrical power transferred to the network $(\mathrm{pu})$.

$P_{m}$ Mechanical power generated by the shaft (pu).

$T_{d o}^{\prime \prime}$ d-axis open circuit sub-transient time constant (sec).

$T_{q o}^{\prime \prime}$ q-axis open circuit sub-transient time constant (sec).

$\theta$ Rotor angle of a generator relative to the COI (rad).

$T_{d o}^{\prime}$ d-axis open circuit transient time constant (sec).

$V_{c r}$ Maximum amount of energy can be absorbed by the post fault system while remaining in synchronism.

$X_{d}^{\prime} \mathrm{d}$-axis transient reactance $(\mathrm{pu})$.

$X_{d}^{\prime \prime} \mathrm{d}$-axis sub-transient reactance $(\mathrm{pu})$.

$X_{d}$ d-axis reactance $(\mathrm{pu})$.

$\xi$ Slack variable.

$X_{l}$ Leakage reactance $(\mathrm{pu})$.

$X_{q}$ q-axis reactance $(\mathrm{pu})$.

$y_{n}$ Output label of data point $x_{n}$. 


\section{Chapter 1}

\section{Introduction}

Modern power systems are compelled to operate close to stability limits due to increasing demand, competition, and social constraints on the expansion of transmission systems. The operation of a power system with small stability margins increases the risk of wide spread and high impact events. Recent major blackouts reported all over the world indicated that the power interruptions resulting from power system instabilities have a significant impact on the entire society and the economy [1-3].

To date, power systems are usually operated based on the margins obtained by off-line studies. Most Energy Management Systems (EMSs) in control centres are capable of performing on-line Static Security Assessment (SSA) to ensure that the

power system can withstand the set of credible contingencies. During the SSA the postfault operating point of the power system under credible contingencies are evaluated for acceptable operating conditions. The Transient Stability Assessment (TSA) examines the dynamic behaviour of the power system for a set of probable events. These studies require a very long time when compared to SSA. The usual practice is to perform off-line TSA for a set of selected operating conditions. A typical study may range from several hours to days depending on the number of operating points, number of contingencies and the duration (simulation time) of the dynamic simulations. Typically the set of operating guidelines are determined based on the results 
of these off-line TSA. Instead of relying on the off-line studies, which are usually based on the worst case assumptions, on-line TSA attempts to determine the system security based on the system and the operating conditions at the time of assessment or using a most recently available system snapshot.

\subsection{Real-time Dynamic Security Assessment (DSA)}

The real-time TSA involves capturing the recent system snapshot and examining the dynamic behaviour of the power system for a set of probable events. As the system conditions evolve with time, the use of conventional TSA techniques produce outdated assessment results due to the longer time taken for the computational cycle. The industry accepted time for a computational cycle is about 15-30 min [4]. However, the results of the security assessment becomes more accurate and relevant when the computational cycle is more frequent. Further, if the actual operating conditions and the stability margins are known, the systems can be operated beyond the worst case operating conditions obtained using the off-line studies (i.e. closer to the known actual stability margins at a particular time). The ability to operate closer to the known stability margins directly contribute to the increase of revenue. For example, if the secure transfer capabilities are known at a particular time, the maximum power export/import can be set to maximize the revenue.

The term on-line TSA is coined for real-time/ near real-time DSA systems. Functions of a typical on-line DSA system, according to [5], can be classified as follows:

- measurements

- modelling

- computation

- reporting and visualisation

- control actions; and 
- other functions

The time consuming numerical computations take place in the computation phase. Invention of faster algorithms for the said phase has been a research topic for many years. This thesis presents a novel approach for faster TSA to be applied for on-line DSA based on the direct methods of stability assessment and machine learning.

\subsection{Classification of power system stability and se- curity}

A typical power system stability assessment study may include several techniques in order to assess various forms of stability.

The stability of a power system in general is defined in [6] as

the ability of an electric power system, for a given initial operating condition, to regain a state of operating equilibrium after being subjected to a physical disturbance, with most system variables bounded so that practically the entire system remains intact.

The potential instabilities in modern power systems can be of various forms. The accurate identification of different types of instabilities leads to broader understanding and effective mitigation of those instabilities. Stability analysis is facilitated by the classification of stability into appropriate categories based on $a$ ) physical nature of resulting instability; $b$ ) the size of disturbance considered; $c$ ) the devices, processes and the time span; and $d$ ) the most appropriate method of calculation and prediction of stability [7].

The physical disturbances that occur in a power system can be classified as small or large. Continuous load changes are considered as a small disturbance. The disturbances such as faults in transmission lines and tripping of large generators are considered as large disturbances. Based on the duration for such an event to result 
in a system instability, the resulting instabilities can be further classified into short term or long term. Practically, the power system stability is assessed with respect to a set of predefined disturbances. These scenarios are selected based on the high probability of occurrence and their impact to the system operation. As in [6], the power system stability can be classified into three main forms:

- Rotor angle stability: refers to the ability of synchronous machines of an interconnected power system to remain in synchronism after being subjected to a disturbance

- Frequency stability: refers to the ability of a power system to maintain steady frequency following a severe system upset resulting in a significant imbalance between generation and load.

- Voltage stability: refers to the ability of a power system to maintain steady voltages at all buses in the system after being subjected to a disturbance from a given initial operating condition.

Rotor angle stability of large disturbances is studied under transient stability. The rotor angle stability under the small disturbances are studied under small-disturbance rotor angle stability. Similarly, voltage stability is studied for large and small magnitude of disturbances.

The type of stability assessment focussed in this thesis is the rotor angle stability under large disturbances. The rotor angle variations of the post disturbed system may result in aperiodic angular separation resulting in system instability. The angular separation resulting in losing synchronism due to insufficient synchronising torque is referred to as first swing instability. The study duration for first swing instability is usually within $3 \mathrm{~s}$ to $5 \mathrm{~s}$ [8]. In addition, in large power systems the instability may occur beyond the first swing. The accepted study duration for multi-swing instabilities is $10 \mathrm{~s}$ to $20 \mathrm{~s}[4,6,9]$. 


\section{Power system security}

The power system security refers to its ability to survive from a set of credible contingencies. The two phases of the stability assessment process are to:

1. check if the postfault power system settles in a stable operating point

2. check if the equipment and voltage ratings are not violated at the postfault operating point

DSA is performed for the set of credible contingencies to see if the postfault power system converges to a stable operating point. An operating point is said to be secure if the system is stable under the set of probable contingencies. Otherwise, the system is considered as insecure. SSA is performed at the postfault operating points to check if the operating point is acceptable in terms of the second phase. SSA requires the steady state power flow of the network. DSA, in addition, requires the dynamic data (model data of dynamic devices), the list of contingencies, relay data (characteristics of the protection data) and the set of variables to monitor. This study usually assumes that the power system is in a steady state prior to the occurrence of the disturbance. The derivation of the set of credible contingencies is influenced by the likelihood of occurrence and the reliability criteria.

The DSA refers in this thesis includes the transient stability analysis of a particular power system operating point subject to a contingency listed in a set of credible

contingencies. This type of study is also called TSA or transient rotor angle stability assessment. Further, it should be noted that the DSA mentioned in this thesis does not include the study of small-signal rotor angle stability.

\subsection{Motivation}

The need and the advantages of being able to accurately monitor the security of a power system, based on the current system topology and the operating conditions, 
have been identified. The long time taken to complete the computational phase is the most significant limiting factor for the successful implementation of on-line DSA systems (A detailed classification of techniques proposed in literature for this problem has been presented in Chapter 2). The development of computationally faster and accurate classifying algorithms enables more frequent TSA cycles and in turn leads to successful implementations of on-line DSA systems. Accordingly, the main motivation of this thesis is to investigate a TSA algorithm which is appropriate for on-line DSA.

A. M. Lyapunov presented a method for stability assessment of a non-linear dynamic system without solving differential equations [10]. Based on this approach, an alternative method called direct method for power system TSA was initially proposed in [11]. In [11], the stability is assessed based on the energy acquired at a particular time which is computed using a function called an energy function. If the energy is less than the critical energy, the system is stable and the system may be unstable otherwise- Lyapunov stability provides only the sufficient conditions for assessing the stability [12]. The critical energy is the amount of energy that the system can absorb without losing synchronism. Direct methods conclude the system stability status by simulating the fault-on power system model up to the fault clearing time. Accordingly, this method demands very less computational effort when compared to the time domain numerical integration, which is the most accurate method of TSA, that requires the simulation of post fault power system model for several seconds. Researchers' interest on direct methods decreased rapidly with the ability to perform faster time domain simulations of power system models due to the increased computational power in the modern computers. Another reason for lack of progress in this direction was due to the fact that analytical energy functions for multi-machine power system models with detailed device models cannot be derived [13]. Further, the assessment accuracy obtained using the direct method of stability assessment using simplified power system models along with simple methods of critical energy computation (Potential Energy Boundary Surface (PEBS) [14]) is insufficient for DSA 
of practical power systems. Energy functions, that are the basis of stability assessment in direct methods, represent the energy acquired by the generators at a given time subsequent to a disturbance. Energy functions represent the acquired energy in terms of physically meaningful quantities: kinetic and potential energies. Although the classical direct methods alone do not qualify as a stand-alone TSA technique, it was motivating to investigate if the theoretically sound information produced by the energy functions within a very short simulation time may serve as valuable features for machine learning based implementations towards faster TSA.

The applicability of machine learning for transient stability assessment has been investigated in literature for DSA and for post disturbance transient stability assessment and has shown promising results in recent literature [15-21]. The approaches presented in [15-20] use databases generated off-line aiming to cover all possible operating conditions and topology changes. However, it is challenging to determine if an initially trained network is valid to assess the stability of a particular system operating state once the system is evolved to a different operating state or a different network topology. On the other hand, producing a single learned network to address the entire set of operating conditions of a power system is most unlikely to succeed. The recently proposed approach in [21] recognizes the significance of frequently updating the classification model and has made provisions to revise the decision tree in use. Another practical difficulty in application of machine learning methods is that these methods require large sets of data generated for training the networks. Although the training data generation can be performed off-line using distributed processing techniques the time spent on data generation process makes these techniques unattractive for on-line DSA. Therefore, the investigation on machine learning based TSA techniques that can perform accurate stability assessments using the networks trained using smaller sized datasets is necessary in order to enable practical implementations of on-line DSA that incorporates machine learning techniques.

It is agreed upon that the key to enable frequent computational cycles in on-line 
DSA is to investigate innovative TSA algorithms. A practical on-line DSA system is an integration of many functional entities. Some of these entities (e.g.: EMS, existing software tools used for off-line data generation) already exist in today's operational power systems. Other functional entities will be newly introduced (e.g.: computational hardware for data generation and machine learning). The implementation requires the seamless integration of these functional units while ensuring the expected performance. These existing entities may have inherent limitations (and/or incompatibilities) to introduce bottlenecks for the entire on-line DSA system. Therefore, it is inappropriate to conclude the performance of an on-line DSA system based only on the algorithmic performance. The most accurate way of assessing the performance of an on-line DSA system is through the implementation. Accordingly, the need for the design and development of an appropriate general purpose framework for testing on-line DSA algorithms has been a motivation for this thesis. In addition, it is understood that the development of data generation algorithms for power system based machine learning applications consumes a significant portion of time of a research or a study. Having a ready-made and a customizable tool is an advantage. The need for the design and development of a stand-alone tool to automate data generation for power system stability studies has been another objective of this research.

\subsection{Research Objectives}

The main objective of the thesis is to propose a novel algorithm to enable frequent computational cycles in on-line DSA by using the techniques of direct method of stability assessment and machine learning. It is expected to investigate the ability to utilize the advantage of direct method, which is to conclude the stability status of the post fault system without explicit numerical integration, of stability assessment in modern on-line DSA systems. Also, in order to make the on-line DSA systems viable for practical implementations, it is expected to investigate the ability to train 
accurately classifying learned networks with small sized datasets.

This overall goal is to be achieved by achieving the following specific objectives:

- Study of proposed approaches for on-line DSA and their performance.

- Development of the direct method of stability assessment algorithms for a test power system model.

- Development of a novel algorithm that uses the outcome of energy functions computed at the fault clearing time as input features to machine learning algorithms.

- Development and implementation of software algorithms to automate data generation for machine learning to enable testing of the proposed algorithm for different power system models, operating conditions and contingencies.

- Investigation of an appropriate configuration of the above algorithm in terms of implementation of on-line DSA.

- Development of an integrated on-line DSA framework for testing the performance of the proposed algorithm in real-time. This is expected to resolve critical data communications aspects, identify the computational hardware requirements; and to identify the capabilities and limitations of the existing tools to be used in automation. The completion of this phase involves: a) the development of the real-time simulation model of the test power system; $b$ ) configuration of the automated data generation software system; c) design of the distributed processing architecture of the final implementation; $d$ ) incorporate the EMS functionality to the real-time simulated test power system.

In addition to the core research objective, following two tools/frameworks are expected to be designed and developed: 
- A general purpose and a stand-alone software system for automating data generation for power system transient stability studies.

- A general purpose framework for testing on-line DSA algorithms.

\subsection{Thesis Overview}

The rest of the thesis is organized as follows. Chapter 1 presents a classification of techniques related to improving the computational performance of the on-line DSA and the background theory of power system modelling for DSA. Chapter 2 presents the relevant theory and application details of direct method for the stability assessment of electrical power systems. Chapter 3 describes the data generation process and the details of the design and the development of the software system for automating the data generation. Chapter 4 presents the novel algorithm proposed for the on-line DSA. Chapter 5 presents the details of the real-time testing of the proposed algorithm along with the design and development details of the framework for testing on-line DSA algorithms. Finally, Chapter 6 presents the conclusions, contributions and the recommendations for future research. 


\section{Chapter 2}

\section{Power System Dynamic Security}

\section{Assessment}

\section{$2.1 \quad$ Introduction}

This chapter presents a survey of approaches presented in literature for fast TSA. Further, the theoretical background of modelling the power system and its components for DSA is discussed.

\subsection{Techniques for fast DSA}

\subsubsection{Machine Learning Techniques}

Machine learning applications for TSA can be for prefault security assessment systems, which is for a pre-fault system to determine the system stability state with respect to an anticipated set of contingencies, or for a fault-on/postfault system to predict the security status with respect to an ongoing disturbance.

The research work presented in [18] proposes the use of an ensemble learning scheme using Extreme Learning Machines (ELMs). ELM proposes a simplified training scheme for single layer feed forward neural networks by randomly selecting inputs 
weights and biases for hidden nodes and analytically determining the output weights via direct calculations. Based on the ELM output, the credibility of the assessment is declared. Next, based on the comparison of the sum of the critical assessments and a user defined threshold for the credibility evaluation, the contingency is classified as credible or incredible. The entire database of past data is used for training. Randomly partitioned sample database is used to train a set of ELMs with randomly selected input features, number of hidden nodes and activation functions. The candidate input features are $P$ load, $Q$ load, voltage magnitude and angle of each load bus, $P, Q$ generation of each generator, total $P$ and $Q$ loads and generation of the entire system. The authors recommend to perform numerical integration for the contingencies classified as incredible. For the IEEE 50 generator system [22], using 6345 operating points generated by varying loads in the range of $20-50 \%$ and and with the randomly generated network topologies for a single contingency, the authors have obtained accurate classification results for $93.85 \%$ of identified credible cases. Authors have observed an accuracy drop when multiple contingencies are introduced. Further, authors have proposed to use three ELMs to extend the assessment to four contingencies. However, the decision to have three ELM ensembles is obtained by a trial and error approach. As mentioned above, the credibility of a classification is decided by comparing to a user specified threshold parameter and that leaves the flexibility to the user but lack of a generalised procedure for the parameter selection may have a significant impact on the performance.

The approach presented in [19], which is for the post fault security assessment, uses an Artificial Neural Network (ANN) to predict the transient instabilities from the Phasor Measurement Unit (PMU) measurements in order to initiate the remedial actions such as load shedding, islanding, generation tripping or a combination of these actions. For the IEEE 39 bus test power system, the authors have proposed an ANN with two hidden layers consisting of 10 and 5 hidden nodes respectively in layer 1 and layer 2. The inputs to the neural network is the rate of change of 
generator bus voltages and angles. Output of the network is a boolean value indicating the instability of the post-fault system by 1 and the stability by 0 . The prediction accuracy is reported as $98 \%$. Although it is mentioned that a $30 \%$ of the simulated cases were used for training, the total number of simulation cases generated were not mentioned in the paper. Further, load and generation deviations simulated during the database generation is not indicated in the paper. Size of the training dataset and the loading changes accommodated successfully are significant in terms of evaluating the applicability of a proposed machine learning algorithm for the on-line security assessments.

The application based on Support Vector Machine (SVM) for the post-fault transient stability prediction is presented in [17]. To enable the predictions for unbalanced faults, the authors has proposed to train a SVM per phase (three SVMs for the three phase system model). For the IEEE 39 bus test power system a SVM was trained using 492 simulation cases generated by creating faults at every transmission line at $25 \%, 50 \%$ and $75 \%$ of the transmission line length. A standard fault clearing time of 5 cycles was applied to all contingencies. The above simulations were done at three increased loading levels (5\%,7\% and 10\%). Over $97 \%$ accuracy was obtained by using four consecutive samples of each generator bus voltage of the post fault system trajectory. The method demands the time domain simulation of four cycles beyond the fault clearance. The number of inputs to the SVM in this case is 40 . The overall prediction accuracy indicates no False Alarms (FAs) but 4.5\% of False Dismissals (FDs).

The research work presented in [23] proposes a continuation of the work presented in [17] by using only a selected set of generator variables as the inputs to the SVM and by using multiple SVMs to support the prediction in selected areas (named as incredible area). Further, authors have attempted to have shorter durations for postfault trajectory simulation. The test results are presented based on the IEEE 39 bus test power system. Number of training cases generated is 1656 . Three phase to 
ground faults were created at each transmission line at three loading levels $(80 \%, 100 \%$ and 120\%). The paper presents two schemes. In both schemes the number of inputs can vary depending on the length of the post-fault simulation. The SVMs generated using power imbalance or the electromagnetic power as the inputs, with a $20 \mathrm{~ms}$ post-fault trajectory simulation, has predicted the stability about $94 \%$ accurately with 60 inputs to the SVM. This accuracy has increased up to $99 \%$ for a $240 \mathrm{~ms}$ post-fault trajectory simulation. However, in that case the number of inputs to the SVM was 250. In the second scheme, the authors propose to have another SVM trained using a combination of generator variables for about $60-80 \mathrm{~ms}$ of post-fault trajectory. Scheme 2 uses a combination of the above mentioned two feature sets as inputs. Therefore, the number of inputs for a SVM in scheme 2 will be doubled as the SVM trained in scheme 1 for the same duration of the post-fault trajectory. Authors propose to consider the vote of the scheme 2 SVM, if the scheme 1 SVM indicates the assessment in the incredible area. As presented, the results indicates some improvement in the classification accuracy and also there is a flexibility in selecting the duration for the post-fault trajectory simulation. However, this method requires a very large set of training data and a large number of inputs to the SVM when compared to the method proposed in [17].

Machine learning techniques have captured researchers attention as a candidate technique for faster on-line DSA. SVMs have indicated better classification accuracy [24] over the multi-layer perceptron networks (such as ANNs). The above discussed approaches, including [16], uses databases generated in off-line aiming to cover all the possible operating conditions and topology changes. The application of data mining techniques to speed up the DSA process by using the historical data from large databases is investigated in [25] and [26]. However, it is challenging to determine if an initially trained network is valid to assess the stability of a particular system state once the system state is evolved to a different operating state or a different network topology. The recently proposed approach in [21] recognizes significance of frequently 
updating the classification model and has made provisions to revise the decision tree in use.

\subsubsection{Direct Assessment Methods}

The research work presented in [27] focuses on the implementation of a method named group based Controlling Unstable Equilibrium Point (CUEP) method which is to determine a common CUEP for a set of contingencies. Therefore, the time spent on the computation of the CUEP becomes less. The authors propose to group the contingencies involving geographically close events to share the same CUEP for stability assessment calculations. This grouping has been done based on rigorous studies of numerical simulations of the particular set of contingencies. The method has been used to classify the contingencies into two categories: a) highly stable cases; and b) unstable/undecided cases. For the marginal stable cases identified above, the authors propose to perform time domain stability analysis. The results are presented for a list of 200 contingencies comprised of 2 unstable, 20 critically stable and 178 stable. The TEPCO-BCU algorithm was able to capture the 2 unstable contingencies accurately. An $86 \%$ of stable cases were classified as stable. The status of classification of the marginally stable cases is not mentioned in the paper. Assuming those were identified as unstable/undecided, altogether $25+20=45$ (out of a 200) cases demands for detailed time domain stability analysis. The main advantage of the proposed method is to have no FDs. The authors have proposed to use parallel processing in order to speed-up the computation process. The authors have also tested the proposed scheme for contingency classification in the PJM Interconnections's Transient Stability Analysis and Control system. The average computational time per contingency is reported as $1.18 s$ on a single node computer. However, the grouping details of contingencies list of 200 contingencies are not reported. According to this method, the number of CUEPs to be calculated depends on the number of groups of contingencies. Therefore, the computational complexity of the algorithm strongly depends on the number 
of contingency groups.

The Second-Kick method, based on the PEBS method, has been developed by BC Hydro as part of it's on-line DSA system [28]. The purpose of the method is to find the Transient Energy Margin (TEM) of a particular contingency by calculating the PEBS crossing point (Calculation of PEBS crossing point is explained in Section 3.5.1). First, after application of the contingency $\left(t=t_{k e m i n 1}\right)$, if the minimum kinetic energy is greater than zero, the system is unstable. If the minimum kinetic energy is zero, the system is stable. If the system is stable at $t=t_{\text {kemin } 1}$, a prolonged fault, called Second Kick, capable of making the system unstable is applied and the simulation is continued for one time step following the fault recovery. The dot product, as mentioned in Equation (3.31), is obtained at the second fault clearing time. A sign change is observed if the PEBS is crossed during that time, therefore, the exact crossing point is obtained using the interpolation, $t=t_{P E B S}$. However, as the network structure during the fault is different to the post-contingency network, the PEBS crossing point obtained is not specific to the post-contingency network, but, that is the upper boundary of the fault to apply. Then, a fault shorter than $t_{P E B S}$ is applied and simulated until a minimum of kinetic energy, $K_{e m i n 2}$, is reached. A minimum of kinetic energy will be observed at the PEBS crossing point.

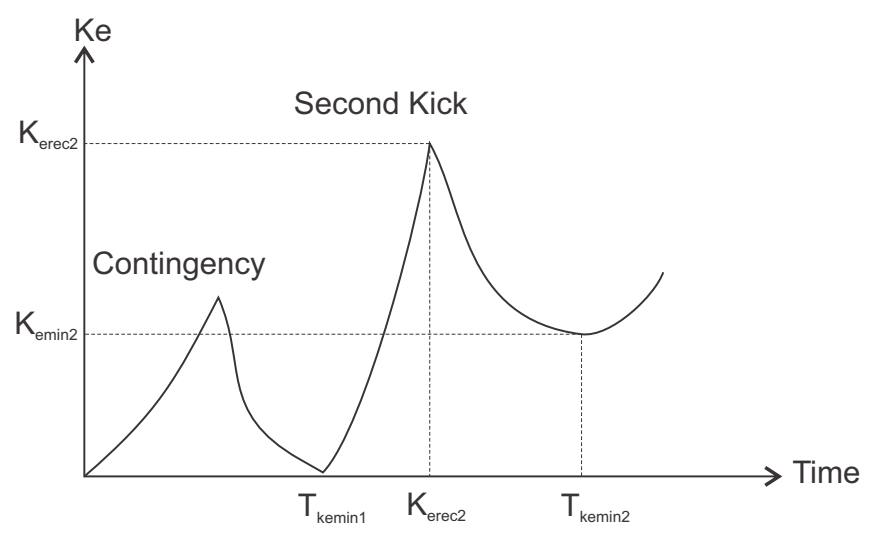

Figure 2-1: Kinetic energy variations of the Second Kick method [29]

Therefore, the TEM is obtained based on the idea that it is obtained by the 
difference of the kinetic energy at the PEBS crossing, $K_{\text {emin } 2}$, and the kinetic energy injected by the Second Kick at the fault clearing time, $K_{\text {erec } 2}$, along with the potential energy change, $D_{P E}$, during the Second Kick, $T E M=K_{\text {erec } 2}-K_{\text {emin } 2}+D_{P E}$ (Figure $2-1)$.

As this research work is reported in 1996, the simulation times taken are not comparable to the modern research due to the speed of the present day computers. The authors have proposed several techniques to enhance the performance of the Second Kick method: a) Kiana method; b) Fast Second Kick method [29]; and c) use of ANN to predict the TEM [30].

The research work reported in [30] is an initial step towards the application of machine learning to predict the TEM which is originally proposed to obtain using extensive time domain simulations as mentioned above in the Second Kick method. The authors propose to use a large number of features, about $57-61$, as the inputs to the ANN. The TEM has been estimated with an average $5 \%$ error when compared to the Second Kick method. The average misclassifications resulted during the estimation of the TEM using the ANN is $4 \%$, the misclassified cases were reported to have an average of $10 \%$ error with the TEM obtained using the Second Kick method. However, the results reported are only for the estimation of the TEM using the ANN, not for predicting the system security state.

A method to calculate the critical energy based on the energy of a critical group of generators is proposed in [31]. The energy function used is obtained by the first integral of motion as mentioned in Equation (3.27). The critical group of generators is the set of generators having a larger rotor angle than the maximum difference between any two rotor angles at the point where the potential energy reaches to a maximum. The authors have tested the concept for a single operating point of IEEE 39 generator system and for a single operating point of a 50 generator system for several contingencies and indicated that the critical clearing time obtained using the energy of the critical group of generators are close to the value obtained using 
the numerical integration than the value obtained using the conventional individual energy function method.

\subsubsection{Functional Representation of Stability Boundary}

For a power system, if a mapping function $f(x)$ can be obtained to uniquely map the current operating point, $x$, to a stability index, $y$, the transient stability or margin can be expressed as a non-linear function of the power system variables. This mapping function, $f(x)$, can not be derived using analytical methods due to the involvement of large number of coupled Differential and Algebraic Equations (DAEs). In [15], the authors have attempted an empirical approximation to $f(x)$ using machine learning techniques along with a non-linear transformation to a higher feature space. The significance is that a non-linear transformation is applied to the original variables: voltage magnitudes; and phase angles, so that the transient stability boundary is approximately linear in terms of the transformed variables. However, the linear function obtained using the transformed variables is non-linear in terms of original variables. The attractiveness of this method is that the estimated function is not a linearised approximation, although a linear estimation method, Kernel Ridge Regression, is used. The authors propose to train a separate learned network for each contingency. The stability assessment results are reported using the IEEE 39 bus test power system. A $95 \%$ of classification accuracy is reported for a network trained using 100 data points. The maximum FDs resulted by the two learned networks is $5 \%$. During the database generation, loads and generation have been randomly perturbed by $10 \%$ (for active and reactive power) and by $2 \%$ for generator reference voltage settings. The resulted power mismatch has been taken care of by the infinite bus. Authors have demonstrated the possibility of increasing the range of deviations up to $20 \%$ (generator voltage magnitude setting was remained at $2 \%$ ) by doubling the size of the dataset. Another advantage is the ability to predict the stability using the pre-fault system variables without performing additional numerical integrations 
or calculations to obtain the input data to the learned network. This method requires the training of a dedicated network for each contingency. Accordingly, it requires a dedicated training dataset for training the networks for each contingency.

The authors of [20] were able to obtain $0 \%$ FDs on the same dataset used in [15] by using the Multi-Step Lasso technique.

\subsubsection{Using Hyperplanes and Nomograms}

A commonly used practice in the industry for stability boundary characterizations is to use a two dimensional graph called a nomogram which is constructed based on two selected critical parameters. In order to develop a nomogram, the selected critical parameter is varied while other critical parameters are set to a selected value. All the other non-critical parameters are set to constant values. Points on the nomogram plot are calculated using multiple computer simulations. This approach for security boundary estimation has several disadvantages including the inaccurate boundary representation, intensive computational burden and a very little possibility in integrating the limits with the EMS. Further, limited security constraints considered in nomograms are incapable of accounting for the coupling effects on a system-wide basis.

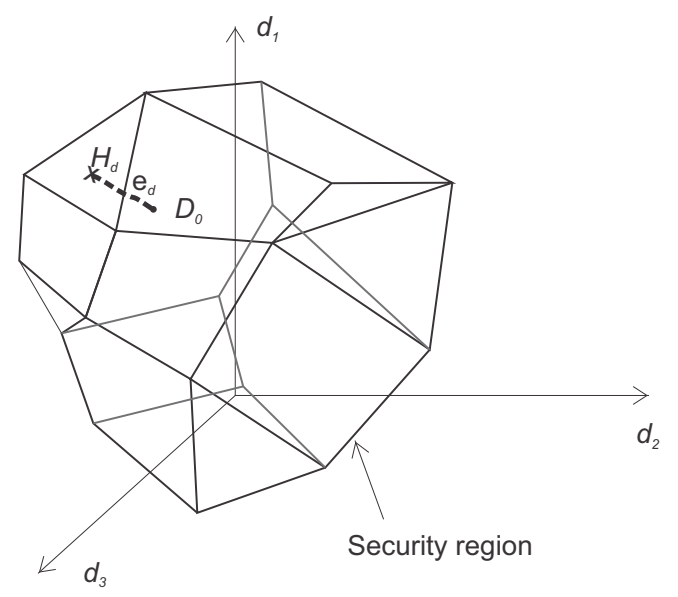

Figure 2-2: Conceptual view of an approximated security region [32] 
Instead, the wide area security region in higher dimensions is an extension to the nomograms to incorporate a number of critical parameters to monitor the system security in a wide area. According to [33], Figure 2-2 shows an example of an approximated security region as a polyhedron where $D_{0}$ is the base operating point, $H_{i}$ is the hyperplane $i$, and $\xi_{i}$ is the stress vector.

In [33], authors proposes to use hyperplanes in multi-dimensional space consisting of system parameters that are critical for security analysis. The authors propose to construct the hyperplanes using off-line simulations reflecting thermal, voltage, voltage stability, small signal or transient stability constraints. Authors follow the approach proposed in [32] to define the following parameters.

1. Control and descriptor parameter spaces: Zonal generation (and loads in emergency cases) can be controlled directly by operators are called control parameters. Transmission path flows depend on control parameters are called descriptor parameters.

2. Stress Direction: the stress direction and the procedure specifies how the system parameters are changed from their base toward the stability boundary as a function of a scalar stress parameter as shown in Equation (2.1).

$$
D=D_{0}+\alpha \Delta D
$$

where $D_{0}$ is the base point in $D$-space, $\Delta D$ defines the stress direction and $\alpha$ is a scalar parameter.

Authors have reported the simulation results for the full size Western Electricity Coordinating Council (WECC) system model which comprise of remedial action schemes and other controls affecting the system behaviour. The simulations were carried out based on 8 descriptor parameters and 9 control parameters. These parameters were selected based on the experience- having the most impact on the system security. Using the simulation results authors point out the significance of the multidimensional 
approach in investigation of coupling effects between critical parameters. Estimation error of the stability margin is evaluated using the difference between: $a$ ) the shortest distance to the security region boundary obtained by starting from the current operating point and and following the stress direction (normal to the hyperplane considered until the intersection point is found), and $b$ ) obtaining the distance to the respective hyperplane using the proposed method. The results demonstrates the feasibility of the developed method. The method can be used to obtain the security margin for significant and small number of parameters such as transfer limits.

\subsubsection{Variable Time-step Integration and Early Termination Methods}

The selection of the appropriate simulation time-step is important in power system dynamic simulations. The algorithms designed to capture system transients using explicit integration formulas and small time steps of integration are inefficient to simulate scenarios spanning in the order of minutes or more. On the other hand, the larger time-steps are incapable of capturing the fast dynamics. Variable time-step integration methods facilitates to alter the time-step on the run based on a local error of an estimated value for a time step and an actual value. If the local error is above a threshold, the program automatically alters the time-step of the integration $[34,35]$. Literature have reported about a 10 times speed up when compared to the fixed time-step integration [36].

\subsubsection{Dynamic Equivalencing Techniques}

Dynamic equivalencing is mainly proposed for two reasons: a) to speed up the computational time for transient stability assessment in large system models, and $b$ ) due to the unavailability of the Supervisory control and data acquisition (SCADA) data of the external systems in large interconnected power networks. [37]. Some commer- 
cial tools are capable of dynamic equivalencing. However, generation of an equivalent model capable of delivering the expected performance for on-line DSA studies is challenging.

For example, TSA of the entire interconnected North American power system (which operates under several operators) has to solve a very large number of differential equations. The model of Midwest Reliability Organization (MRO) system, where Manitoba Hydro is included, has more than 50,000 buses. It can take hours to complete a DSA. Therefore, the computational cycle time is inappropriate for power systems monitoring. The usual practice is to use the system equivalents at the boundary buses of a particular operator or the study area. Manitoba Hydro uses a buffer zone and the system equivalents are used at the boundary of the buffer zone and the external system. The selection of an appropriate equivalent circuit plays a key role towards the accuracy of the outcome of a particular study. The equivalent circuits are used in rotor angle TSA for which the studies about the electromechanical oscillations typically are in the range of $0-2 \mathrm{~Hz}$ [38], are also referred to as Low-Frequency Equivalents (LFEs).

The three main approaches as stated in [38] to obtain a LFE are:

1. approximate using linear models (modal methods)

2. identification and representation of coherent generator groups using equivalent generators, and

3. measurement or simulation based methods to approximate the model parameters of a large system

In literature, dynamic equivalencing is also proposed in relation to the Equal Area Criterion (EAC). The idea behind the EAC was further extended to multi machine power systems through the introduction of the Extended Equal Area Criterion (EEAC). The EEAC is based on the notion that transient instability is a result of the separation of generator rotor angles into two clusters after being subjected to a 
severe transient disturbance. These two sets of generators are transformed into an equivalent two machine system, one machine representing the dynamics of the critical cluster and the other representing the dynamics of the remaining cluster of machines. Finally, the equivalent two-machine system is reduced to a Single Machine to Infinite Bus (SMIB) system for which the EAC can be applied to estimate the transient stability margin [39]. EEAC formulation uses the classical generator model. Since it was originally proposed, many variations have been proposed in literature to enhance the performance of the EEAC method and has been implemented in various DSA applications in its original form or including modifications [36, 40,41].

\subsubsection{High Performance Computing}

Distributed and parallel processing has become an essential technique in modern online DSA systems [36, 42-46]. This technique can effectively accomplish significant speed improvement as the TSA usually includes the assessment of many contingencies. In [43], the authors have reported a significant and almost linear reduction of the computation time by using the parallel processing in multi-core processors.

The utilization of multiple core processors along with simulation decomposition and localisation is proposed for faster processing in [46]. In large power systems, the effects of some disturbances may only be local. The reported work also investigates the possibility of partitioning the DAE solution into several stand-alone solutions. However, it is challenging to propose a general criteria for localisation and partitioning.

\subsubsection{Other Approaches}

Although the requirements of the on-line DSA demands for the faster computations, to date, some large interconnected power system models include dynamic models that demand small integration time-steps to the maintain numerical stability of the 
simulation tools in use. If these models are revised to ensure the numerical stability during simulations, the simulations can be made faster by using larger time steps [4].

The use of look up table approach avoids performing any simulations using real time conditions to obtain stability limits. In this approach, stability limits are calculated by extensive off-line simulations to address the possible operating conditions. These limits are stored in a database and accessed based on similarity of the on-line

operating conditions obtained from the real power system in operations [43]. This is obviously a very fast method to obtain the stability limits as it requires no additional simulations or calculations. However, this method is subjected to two shortcomings: a) if the real time conditions are not matched appropriately, there will be no use of the system, and $b$ ) off-line stability limits calculated are often more conservative than the stability limits calculated on-line according to the actual operating conditions, therefore, the stability margins obtained will be conservative. This would result limiting the benefits of the on-line DSA.

\subsection{Modelling of Power System Components for DSA}

The power system component models are to be selected depending on the type of the targeted study or the analysis. The dynamic phenomena studies can be divided into three categories as in [38].

1. high-frequency transients studies - includes frequency dependant transmission line models and detailed generator models

2. low-frequency electromechanical oscillations studies - transmission lines as constant admittances and generator dynamics excluding stator and network transients

3. sub-synchronous oscillation studies - turbine-generator dynamics and network 
transients need to be modelled adequately

DSA based on time domain TSA belongs to the second category. The interested range of electromechanical oscillations is approximately in the range of $0-2 \mathrm{~Hz}$. The network transients occur typically at very high frequencies. Therefore, the network transients and the stator transients of the synchronous machines are ignored during modelling the power system for electromechanical studies. Accordingly, the transmission network is modelled using admittances calculated at the fundamental frequency. Generators and the auxiliary controllers are modelled using first order DAEs in the following form [38]

$$
\begin{aligned}
\dot{x}_{k} & =f\left(x_{k}, V_{k}, u_{k}\right) \\
I_{k} & =g\left(x_{k}, V_{k}\right)
\end{aligned}
$$

where,

$x_{k}$ : state vector of the $k^{\text {th }}$ device

$V_{k}: R$ and $I$ components of bus voltage

$u_{k}$ : input vector of the $k^{t h}$ device

The following subsections presents the device models that are used for simulations in this thesis.

\subsubsection{Synchronous Generator Models}

\section{Fifth order model}

This synchronous generator model includes five differential equations to represent a) rotor dynamics using the swing equation $(\delta$ and $\Delta \omega) b)$ flux variations of the rotor field winding $\left(\psi_{f d}\right) c$ ) flux variations of two rotor damper windings $\left(\psi_{1 d}\right.$ and $\left.\psi_{1 q}\right)$. 
For simplification, the $\omega_{r}$ in the stator voltage equations are assumed equal to $1 \mathrm{pu}$. This assumes that the effect of the speed variations have negligible effect on stator voltage. The resultant set of first order differential equations are $(2.4,2.5,2.6,2.7$, and 2.8)

$$
\begin{aligned}
\frac{d}{d t} \psi_{f d} & =\omega_{0}\left[e_{f d}-\frac{R_{f d}}{L_{f d}} \psi_{f d}+\frac{R_{f d}}{L_{f d}} L_{a d}^{\prime \prime}\left(-i_{d}+\frac{\psi_{f d}}{L_{f d}}+\frac{\psi_{1 d}}{L_{1 d}}\right)\right] \\
\frac{d}{d t} \psi_{1 d} & =\omega_{0}\left[-\frac{R_{1 d}}{L_{1 d}} \psi_{f d}+\frac{R_{1 d}}{L_{1 d}} L_{a d}^{\prime \prime}\left(-i_{d}+\frac{\psi_{f d}}{L_{f d}}+\frac{\psi_{1 d}}{L_{1 d}}\right)\right] \\
\frac{d}{d t} \psi_{1 q} & =\omega_{0}\left[-\frac{R_{1 q}}{L_{1 q}} \psi_{1 q}+\frac{R_{1 q}}{L_{1 q}} L_{a q}^{\prime \prime}\left(-i_{q}+\frac{\psi_{1 q}}{L_{1 q}}\right)\right] \\
\frac{d}{d t} \Delta \omega_{r} & =\frac{1}{2 H}\left(T_{m}-e_{d} i_{d}-e_{q} i_{q}-k_{D} \Delta \omega_{r}\right) \\
\frac{d}{d t} \delta & =\omega_{0} \Delta \omega_{r}
\end{aligned}
$$

Where

$\omega_{0}:$ synchronous speed in electrical $\mathrm{rad} / \mathrm{s}$

$\Delta \omega_{r}:$ rotor speed deviation $(\mathrm{pu})$

$\psi_{f d}, \psi_{1 d}, \psi_{1 q}$ : flux in the field winding, $\mathrm{d}$ axis damper winding and $\mathrm{q}$ axis damper winding (pu)

$T_{m}$ : mechanical torque $(\mathrm{pu})$

$H$ : inertia constant $(\mathrm{MW} \cdot \mathrm{s} / \mathrm{MVA})$

$K_{d}$ : damping coefficient $(\mathrm{pu})$

$e_{d}, e_{q}: \mathrm{d}$ and $\mathrm{q}$ axis armature voltages $(\mathrm{pu})$

$i_{d}, i_{q}: \mathrm{d}$ and $\mathrm{q}$ axis armature currents $(\mathrm{pu})$

$e_{f d}$ : field voltage $(\mathrm{pu})$ 
$R_{f d}, R_{1 d}, R_{1 q}$ : resistances of field winding, $\mathrm{d}$ axis damper winding and $\mathrm{q}$ axis damper winding (pu)

$L_{f d}, L_{1 d}, L_{1 q}$ : inductances of field winding, d axis damper winding and q axis damper winding $(\mathrm{pu})$

$L_{a d}^{\prime \prime}, L_{a q}^{\prime \prime}$ : subtransient mutual inductances of d and q axis (pu)

$t$ : time $(\mathrm{s})$

The initial conditions are calculated using the network power flow solution.

\section{Classical generator model}

This simplified model is used in studies where the focus is on the first swing stability analysis. In large power system models, some generators are modelled using the classical generator model due to the unavailability of the generator parameters. The foundational work on direct method of stability assessment of power systems was based on the classical generator model. The model is derived based on the following assumptions a) machines are modelled using the constant flux linkage model b) mechanical damping of the rotor is neglected $c$ ) the effects of generator auxiliary controls are neglected.

\section{Generator auxiliary controls}

Generator auxiliary controllers mainly include the excitation system and the Power System Stabilizers (PSSs). An exciter may have a number of control, limiting and protective functions. The axillary controllers contribute to maintain the required performance of the synchronous generator and the power system. The main purpose of an exciter is to control the field current of the synchronous generator to maintain the terminal voltage during the load changes and transients periods [7]. In addition it is expected to provide limiting and protective functions to prevent damage to power 
system equipments including itself. The functions of a given excitation system may depend on the type of the exciter and the application requirements.

PSSs are usually designed based on the small signal analysis to damp out generator rotor oscillations. This is achieved by modulating the generator excitation system reference voltage level.

\subsubsection{Transmission Line Model}

Transmission lines are modelled as an equivalent $\pi$ network between network buses which comprised of a series impedance $(R+j X)$ and two admittance branches representing the line's capacitive admittance $(j B / 2)$. The reactance and the susceptance values are calculated at the nominal frequency.

\subsubsection{Load Models}

Modelling load characteristics is a difficult task due to $a$ ) the existence of many load

types $b$ ) unavailability of load composition information c) changing composition of loads depending on the time of the day, seasons, etc. The extent of the load model complexity depends on the magnitude of the effect of the response of that particular component during a particular study [47].

\section{Constant Admittance Model}

This is a static load model and the power varies directly with the square of the voltage magnitude. This load model is usually used for in the first swing TSA. The use of this model allows for convenient network reduction by incorporating the loads into the transmission network. The early work on theoretical foundations on the applications of direct method of stability assessment for power systems is performed using the constant admittance load model. 


\section{Dynamic Load Model}

It is recommended in [47] to typically have $50 \%$ to $70 \%$ of load with motor models. The motor models are to be based on typical induction motor data. Further, it is not necessary to include dynamic motors in every bus load, so long as the desired portion of total load in an area is represented as motors. Accordingly, a dynamic load model representing induction motors, lighting, and other types of equipment is selected for simulations.

\subsubsection{Interfacing Components}

With the use of constant admittance load model and the classical generator model, the interfacing of loads and generators and the transmission network is straightforward. The loads are converted to constant admittances and then incorporated into the transmission network as shunt admittances. Similarly, generator reactants are also incorporated into transmission network. Then the network is reduced to the generator buses. Initial conditions are computed using the power flow solution of the prefault system. Numerical integration of the set of differential equations is used to obtain the values of current injections of generators for the next time step [7].

When the generators are modelled using $d-q$ references, the set of differential equations corresponding to each generator and it's auxiliary controllers are modelled in it's own $d-q$ reference frame. At each time step, those values in $d-q$ reference frames are converted to the common $R-I$ frame for interfacing. The transmission network is reduced to the buses connecting dynamic devices (i.e. synchronous generators, dynamic loads, Flexible Alternative Current Transmission System (FACTS) devices, etc.) [7]. 


\subsection{Concluding Remarks}

The approaches presented in recent literature for fast TSA were discussed under 8 categories. Further, the theoretical background of modelling the power system and its components for DSA is discussed. 


\section{Chapter 3}

\section{Direct Methods for Power System}

\section{Stability Assessment}

\subsection{Introduction}

This chapter presents the background theory and information on the non-linear systems and the direct method of stability assessment in general and in relation to the power systems. The Transient Energy Function (TEF) and the significance of numerical energy functions are discussed. A set of widely used critical energy computation methods are discussed. The computational details of the assessment algorithms are illustrated using numerical simulations. The objective of this chapter is to provide the necessary theoretical background for the TSA algorithm proposed in this thesis.

\subsubsection{Introduction to Direct Methods of Stability Assess- ment}

Lyapunov presented two methods for stability analysis of non-linear dynamical systems [10].

1. a method to examine the local stability of a non-linear system around an Stable 
Equilibrium Point (SEP) using the properties of it's linear approximation.

2. a method for stability assessment without solving the differential equations of the system. This method is referred to as the direct method.

The principle behind the Lyapunov's direct method is a mathematical extension to the relationship between the total energy of a mechanical system and its stability. If the total energy in a mechanical system is continuously dissipating, the system must eventually settle down to an equilibrium point [12].

\subsubsection{Stability in the Sense of Lyapunov}

Let $B_{R}$ and $B_{r}$ be spherical regions (or balls) defined respectively by $\|x\|<R$ and $\|x\|<r$ in the state space. Let $S_{R}$ and $S_{r}$ be spheres defined respectively by $\|x\|=R$ and $\|x\|=r$. The equilibrium state $x=0$ is said to be stable in the

sense of Lyapunov, if for any $R>0$, there exists $r>0$ such that if $\|x(0)\|<r$, then $\|x\|<R$ for all $t \geq 0$. Otherwise, the equilibrium point is unstable. This definition can be represented as follows.

$$
\forall R>0, \exists r>0,\|x(0)\| \in B_{r} \Rightarrow \forall t \geq 0,\|x(t)\|<R .
$$

Further, the equilibrium point is said to be asymptotically stable if it is stable, and there exist $r>0$ such that $\|x(0)\|<r$ implies that $x(t) \rightarrow 0$ as $t \rightarrow \infty$. An equilibrium point that is stable in the sense of Lyapunov but is not asymptotically stable is called marginally stable equilibrium point (Figure 3-1). If the asymptotic stability holds for any initial state, then the equilibrium point is said to be globally asymptotically stable [12]. 

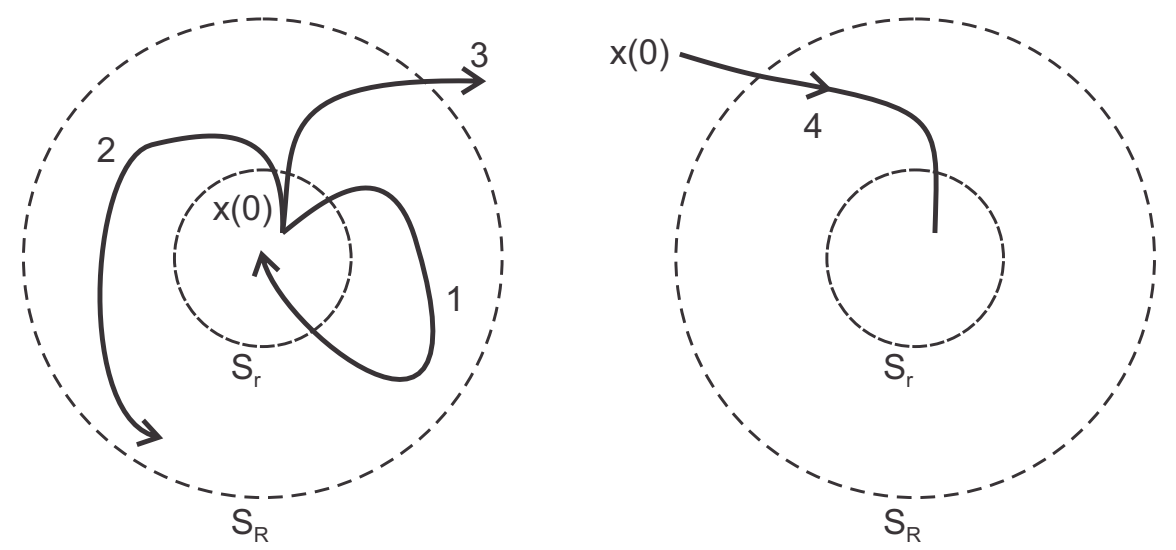

Figure 3-1: Concepts of stability (1: asymptotically stable, 2: marginally stable, 3: unstable, 4 : globally stable) [12]

\subsubsection{Lyapunov Function}

A function $V(x)$ is said to be a Lyapunov function for a given system if in a ball $B_{R}$, the function $V(x)$ is positive definite, has continuous partial derivatives, and its time derivative along any state trajectory of the system is negative semi-definite, i.e., $\dot{V}(x) \leq 0$. The Lyapunov function is also called the energy function.

A scalar continuous function $V(x)$ is said to be locally positive definite if $V(0)=0$ and in a ball $B_{R 0}, x \neq 0 \Rightarrow V(x)>0$. Further, if the above properties hold over the whole state space, then $V(x)$ is said to be globally positive definite. The prefix semi is used to reflect the possibility of a function being equal to zero for $x \neq 0$.

There is no systematic way of constructing Lyapunov functions for general nonlinear systems. This is a fundamental drawback of the direct method. It often requires experience, intuition, trial and error and physical insights to search for an appropriate Lyapunov function. Lyapunov stability theory only provides sufficient conditions for stability. If for a particular Lyapunov function candidate, $V$, the required conditions on the derivative of $V, \dot{V}$, are not met, then conclusions regarding the stability or instability of the equilibrium point can not be drawn [9]. 


\subsubsection{Local and Global Stability Using the Lyapunov Theo- rem}

If in a ball $B_{R 0}$, there exists a scalar function $V(x)$ with continuous partial derivatives such that

- $V(x)$ is positive definite (locally in $B_{R 0}$ )

- $\dot{V}(x)$ negative semi-definite (locally in $B_{R 0}$ )

then the equilibrium point is stable. Further, if the derivative $\dot{V}(x)$ is locally negative definite in $B_{R O}$, then the stability is asymptotic.

In order to assert global asymptotic stability, radial unboundedness $(V(x) \rightarrow \infty$ as $\|x\| \rightarrow \infty$ ) of the function $V$ has to be ensured in addition to expanding the local asymptotic stability to the entire state space. Radial unboundedness ensures that the contour curves of constant energy level correspond to closed curves. It is possible for the state trajectories to drift away from the equilibrium point if the curves are not closed. The following figure illustrates the divergence of the state trajectories while moving towards lower energy levels in a second order system.

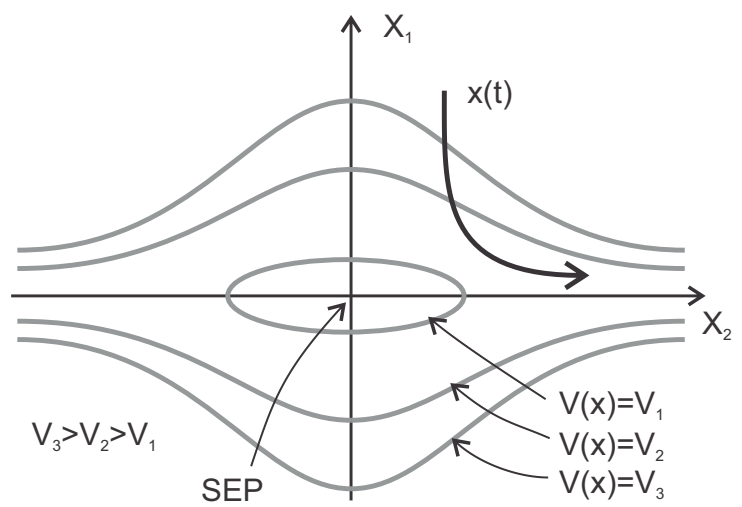

Figure 3-2: Drift of trajectories away from the SEP when the radial unboundedness not assured

Therefore, for a scalar function $V$ of the state $\mathrm{x}$ with continuous first order derivatives such that 
- $V(x)$ is positive definite

- $\dot{V}(x)$ negative definite

- $V(x) \rightarrow \infty$ as $\|x\| \rightarrow \infty$

the equilibrium point at the origin is globally asymptotically stable [12].

\subsubsection{Stability Region of an SEP}

For an SEP, $x_{s}$, there exists a number $\varepsilon>0$ such that every point in the set, $\left\|x_{0}-x_{s}\right\|<\varepsilon$ implies that the trajectory starting from the initial point $x_{0}$ converges to the SEP $x_{s}$; that is, $\phi_{i}\left(x_{0}\right) \rightarrow x_{s}$ as $(t \rightarrow \infty)$. If $\varepsilon$ is arbitrarily large, then $x_{s}$ is called a global SEP.

As the time evolves, every trajectory in the stability region approaches the SEP, $x_{s}$, and every trajectory on the stability boundary evolves on the boundary and approaches the equilibrium points (Unstable Equilibrium Points (UEPs)) on the stability boundary(figure 3-3). The dimension of the stability region is $n$, which is the order of the state space [9].

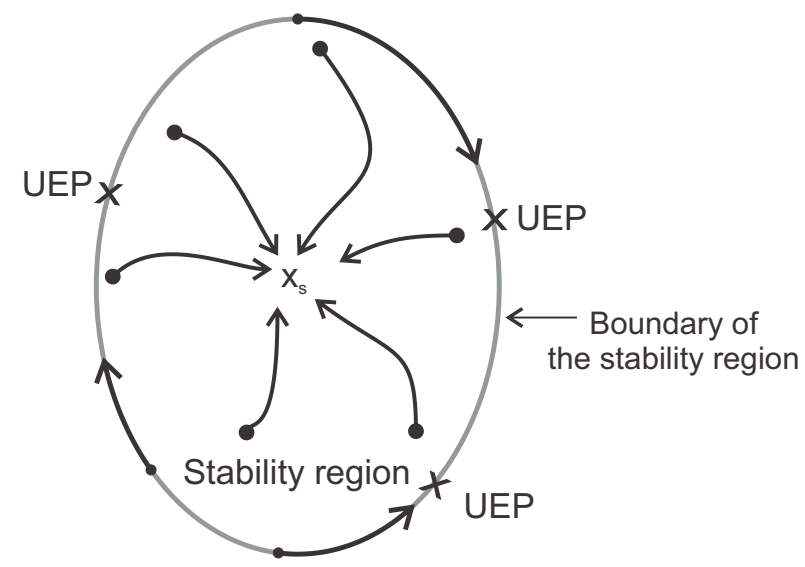

Figure 3-3: Evolution of trajectories within the stability boundary and on the stability boundary [9] 


\subsection{Application of Direct Methods to Power Sys- tems}

An autonomous non-linear system can be represented by a set of non-linear differential equations in the following form:

$$
\dot{x}=f(x)
$$

where $f$ is an $n \times 1$ non-linear vector function and $x$ is an $n \times 1$ state vector. A state $x^{\star}$ is an equilibrium point of the system if $x(t)=x^{\star}$ and it remains equal to $x^{\star}$ for all future time. An equilibrium point can be found by solving the non-linear algebraic equation shown in Equation (3.3). Usually, the equilibrium point is transferred to the origin of the state space.

$$
0=f(x)
$$

Based on the model proposed in Equation (3.2), a power system (represented using the simplified generator model) undergoing a disturbance can be described by the following three sets of differential equations:

$$
\begin{aligned}
& \dot{x}(t)=f_{\text {prefault }}(x) \quad-\infty<t \leq 0 \\
& \dot{x}(t)=f_{\text {faulted }}(x) \quad 0<t \leq t_{c l} \\
& \dot{x}(t)=f_{\text {postfault }}(x) \quad t_{c l}<t<\infty
\end{aligned}
$$

In a practical power system, many events may occur between $t=0$ to $t=t_{c l}$. For simplicity, no structural changes are assumed during the faulted time. The prefault operating point is assumed to be in a steady state. Prefault operating point becomes the initial conditions for the evolving faulted trajectories. Similarly, the faulted tra- 
jectory at $t=t_{c l}$ provides the initial conditions for the postfault trajectory. Stability assessment of the contingency involves the accurate identification of the location of the initial point of the postfault trajectory in terms of inside or outside the region of attraction of the postfault SEP, assuming there exists an SEP for the postfault system.

\subsubsection{TEF}

For the multi-machine power system represented by the classical generator model and the constant admittance load model, the motion of generators are governed by the Equation (3.7) [48].

$$
\frac{2 H_{i}}{\omega_{s}} \frac{d^{2} \delta_{i}}{d t^{2}}+D_{i} \frac{d \delta_{i}}{d t}=P_{m i}-P_{e i}, i=1, \ldots, m
$$

where

$$
\begin{array}{r}
P_{e i}=E_{i}^{2} G_{i i}+\sum_{j=1, j \neq i}^{m}\left(C_{i j} \sin \delta_{i j}+D_{i j} \cos \delta_{i j}\right) \\
C_{i j}=E_{i} E_{j} B_{i j} \\
D_{i j}=E_{i} E_{j} G_{i j}
\end{array}
$$

Denoting $\frac{2 H}{\omega_{s}}=M$ and $P_{i}=P_{m i}-E_{i}^{2} G_{i i}$, Equation (3.7) becomes,

$$
M_{i} \frac{d^{2} \delta_{i}}{d t^{2}}+D_{i} \frac{d \delta}{d t}=P_{i}-\sum_{j=1, j \neq i}^{m}\left(C_{i j} \sin \delta_{i j}+D_{i j} \cos \delta_{i j}\right)
$$

Let $\alpha_{i}$ be the rotor angle with respect to a fixed reference. Then $\delta_{i}=\alpha_{i}-\omega_{s} t$.

$\dot{\delta}_{i}=\frac{d \alpha_{i}}{d t}-\omega_{s}=\omega_{r i}-\omega_{s}$ where $\omega_{r i}$ is the rotor angular speed and the $\omega_{s}$ is the synchronous speed in radians per second. Denoting $\omega_{i}=\omega_{r i}-\omega_{s}$, the Equation (3.24) is represented in first-order differential equations with the state variables $\delta_{i}$ and $\omega_{i}$. 


$$
\begin{aligned}
\dot{\delta}_{i}= & \omega_{i} \\
\dot{\omega}_{i}= & \frac{1}{M_{i}}\left(P_{i}-P_{e i}\left(\delta_{1}, \ldots, \delta_{m}\right)-D_{i}\left(\omega_{i}\right)\right) \\
& i=1, \ldots, m
\end{aligned}
$$

The classical TEF has been proposed based on the power system model represented with respective to the Center of Inertia (COI). Further, the said formulation ignores the mechanical damping $(D)$ of the generator. The COI represents the mean motion of the system. The generator rotor angles and the speeds are referred to the mean motion represented by the angle and the speed of the COI.

The COI is defined as:

$$
\delta_{0}=\frac{1}{M_{T}} \sum_{i=1}^{m} M_{i} \delta_{i}
$$

where

$$
M_{T}=\sum_{i=1}^{m} M_{i}
$$

accordingly, the center of speed is defined as:

$$
\omega_{0}=\frac{1}{M_{T}} \sum_{i=1}^{m} M_{i} \omega_{i}
$$

The transformation of the generator rotor angles and the generator angular speeds to the COI leads to the following two equations. $\theta_{i}$ and $\tilde{\omega}_{i}$ are the rotor angle and the angular speed of the $i^{\text {th }}$ generator with reference to the COI.

$$
\theta_{i}=\delta_{i}-\delta_{0}, \tilde{\omega}_{i}=\dot{\theta}_{i}=\omega_{i}-\omega_{0}
$$

The motion of the COI is defined as:

$$
M_{T} \dot{\omega}_{0}=\sum_{i=1}^{m}\left(P_{m i}-P_{e i}\right)
$$


Further simplification leads to:

$$
\begin{aligned}
M_{T} \dot{\omega}_{0} & =\sum_{i=1}^{m} P_{m i}-\sum_{i=1}^{m} P_{e i} \\
& =\sum_{i=1}^{m} P_{m i}-\sum_{i=1}^{m} E_{i}^{2} G_{i i}-\sum_{i=1}^{m} \sum_{j=1, j \neq i}^{m}\left(C_{i j} \sin \delta_{i j}+D_{i j} \cos \delta_{i j}\right) \\
& =\sum_{i=1}^{m} P_{i}-\sum_{i=1}^{m} \sum_{j=1, j \neq i}^{m}\left(C_{i j} \sin \delta_{i j}+D_{i j} \cos \delta_{i j}\right)
\end{aligned}
$$

Using the relationships $C_{i j}=C_{j i}, D_{i j}=D_{j i}, \sin (-\theta)=-\sin (\theta)$, and $\cos (-\theta)=\cos (\theta)$ :

$$
\begin{aligned}
M_{T} \dot{\omega}_{0} & =\sum_{i=1}^{m} P_{i}-2 \sum_{i=1}^{m-1} \sum_{j=i+1}^{m} D_{i j} \cos \delta_{i j} \triangleq P_{c o i} \\
\frac{d^{2} \delta_{0}}{d t^{2}} & =\frac{1}{M_{T}} P_{C O I}
\end{aligned}
$$

Transformation of Equation (3.7) to refer to the COI by using Equation (3.16) and by ignoring the mechanical damping $\left(\delta_{i j}=\delta_{i}-\delta_{j}=\left(\theta_{i}+\delta_{0}\right)-\left(\theta_{j}+\delta_{0}\right)=\theta_{i}-\theta_{j} \triangleq \theta_{i j}\right)$ :

$$
\begin{aligned}
M_{i} \frac{d^{2}}{d t^{2}}\left(\theta_{i}+\delta_{0}\right) & =P_{i}-\sum_{j=1, j \neq i}^{m}\left(C_{i j} \sin \theta_{i j}+D_{i j} \cos \theta_{i j}\right) \\
M_{i} \frac{d^{2} \theta_{i}}{d t^{2}} & =P_{i}-\sum_{j=1, j \neq i}^{m}\left(C_{i j} \sin \theta_{i j}+D_{i j} \cos \theta_{i j}\right)-\frac{M_{i}}{M_{T}} P_{C O I}
\end{aligned}
$$

The TEF is derived by obtaining the energy integral $[49,50]$ by multiplying the $i^{\text {th }}$ post-fault system Equation $(3.24)$ by $\dot{\theta}_{i}$

$$
V_{i}=\left[M_{i} \dot{\tilde{\omega}}_{i}-P_{i}+P_{e i}+\frac{M_{i}}{M_{T}} P_{C O I}\right] \dot{\theta}_{i}, \quad i=1, \ldots, m
$$


Integrating Equation (3.25) using a lower limit $t=t_{s}$, where $\tilde{\omega}\left(t_{s}\right)=0$ and $\theta\left(t_{s}\right)=\theta^{s}$ is the SEP, results in Equation (3.26).

$$
\begin{aligned}
V_{i}= & \frac{1}{2} M_{i} \tilde{\omega}_{i}^{2}-P_{i}\left(\theta_{i}-\theta_{i}^{s}\right)+\sum_{j=1, j \neq i}^{m} C_{i j} \int_{\theta_{j}^{s}}^{\theta_{i}} \sin \theta_{i j} d \theta_{i} \\
& +\sum_{j=1, j \neq i}^{m} D_{i j} \int_{\theta_{j}^{s}}^{\theta_{i}} \cos \theta_{i j} d \theta_{i}+\frac{M_{i}}{M_{T}} \int_{\theta_{i}^{s}}^{\theta_{i}} P_{C O I} d \theta_{i} \\
& i=1, \ldots, m
\end{aligned}
$$

For a multi-machine system, the energy function of the first integral of the motion is obtained as in Equation (3.27) [48].

$$
\begin{aligned}
V(\theta, \tilde{\omega})= & \frac{1}{2} \underbrace{\sum_{i=1}^{m} M_{i} \tilde{\omega}_{i}^{2}}_{K E}-\underbrace{\sum_{i=1}^{m} P_{i}\left(\theta_{i}-\theta_{i}^{s}\right)}_{P E 1}-\underbrace{\sum_{i=1}^{m-1} \sum_{j=i+1}^{m}\left[C_{i j}\left(\cos \theta_{i j}-\cos \theta_{i j}^{s}\right)\right.}_{P E 2}] \\
& +\underbrace{\sum_{i=1}^{m-1} \sum_{j=i+1}^{m}\left[\int_{\theta_{i}^{s}+\theta_{j}^{s}}^{\theta_{i}+\theta_{j}} D_{i j} \cos \theta_{i j} d\left(\theta_{i}+\theta_{j}\right)\right]}_{P E 3} \\
= & V_{K E}(\tilde{\omega})+V_{P E}(\theta)
\end{aligned}
$$

The steps for deriving the Equation (3.27) are given in Appendix A by using a 3-bus power system model as an example.

The terms in Equation (3.27) are interpreted in [51] as follows:

- $K E$ : total change in rotor kinetic energy relative to $\mathrm{COI}$,

- PE1: total change in rotor potential energy relative to COI,

- PE2: total change in magnetic stored energy of network branches; and

- PE3: total change in dissipated energy of network branches.

The sum of PE1, PE2 and PE3 is referred to as potential energy. 
Equation (3.27) contains path dependant integral terms. Since, the positive definiteness of $V(\theta, \omega)$ is not guaranteed, Equation (3.27) is not a candidate Lyapunov function for the system in Equation (3.24). If $D_{i j} \equiv 0$ (i.e., transfer conductances of network Y matrix are ignored), it is shown that $V(\theta, \omega)$ constitutes a proper Lyapunov Function [48]. According to Equation (3.10), $V(\theta, \omega)$ becomes a candidate Lyapunov function if the network losses are ignored. Although many attempts were made so far, there is no analytical energy function derived for multi-machine power systems with lossy networks [13]. The existence of a candidate Lyapunov function (CLF) for an SMIB system with transmission network modelled with losses has been proven in [50].

\subsection{Numerical Energy Functions}

Numerical energy functions consists of two major terms: analytical terms (path independent terms) and numerical (path dependant) terms. Path dependant terms require numerical approximations. In Equation (3.27), terms $K E, P E 1$ and $P E 2$ are analytically solvable terms. The term PE3 requires a numerical solution. Numerical energy functions were introduced to resolve the difficulty of constructing analytical energy functions for the lossy power system stability models. Path dependant terms can be evaluated using trapezoidal approximation or ray approximation $[9,52]$

\subsection{Stability Assessment}

\subsubsection{Steps for Stability Assessment Using the Direct Method}

1. Numerically simulate the fault-on trajectory

2. Compute the initial point of the postfault trajectory

3. Construct an energy function for the postfault system (Section 3.2.1 and Section 
4. Compute the energy function value at the initial point of the postfault system, V

5. Compute the critical energy for the fault-on trajectory, $V_{c r}$ (Section 3.5)

6. If $V<V_{c r}$, the system is stable, otherwise, the system may be unstable

Figure 3-4 shows the variation of the total and the potential energies of a fault-on trajectory obtained using the TEF. This Matlab simulation is performed on the IEEE 3 generator 9 bus test power system model [53] for a three phase to ground fault at bus 7 .

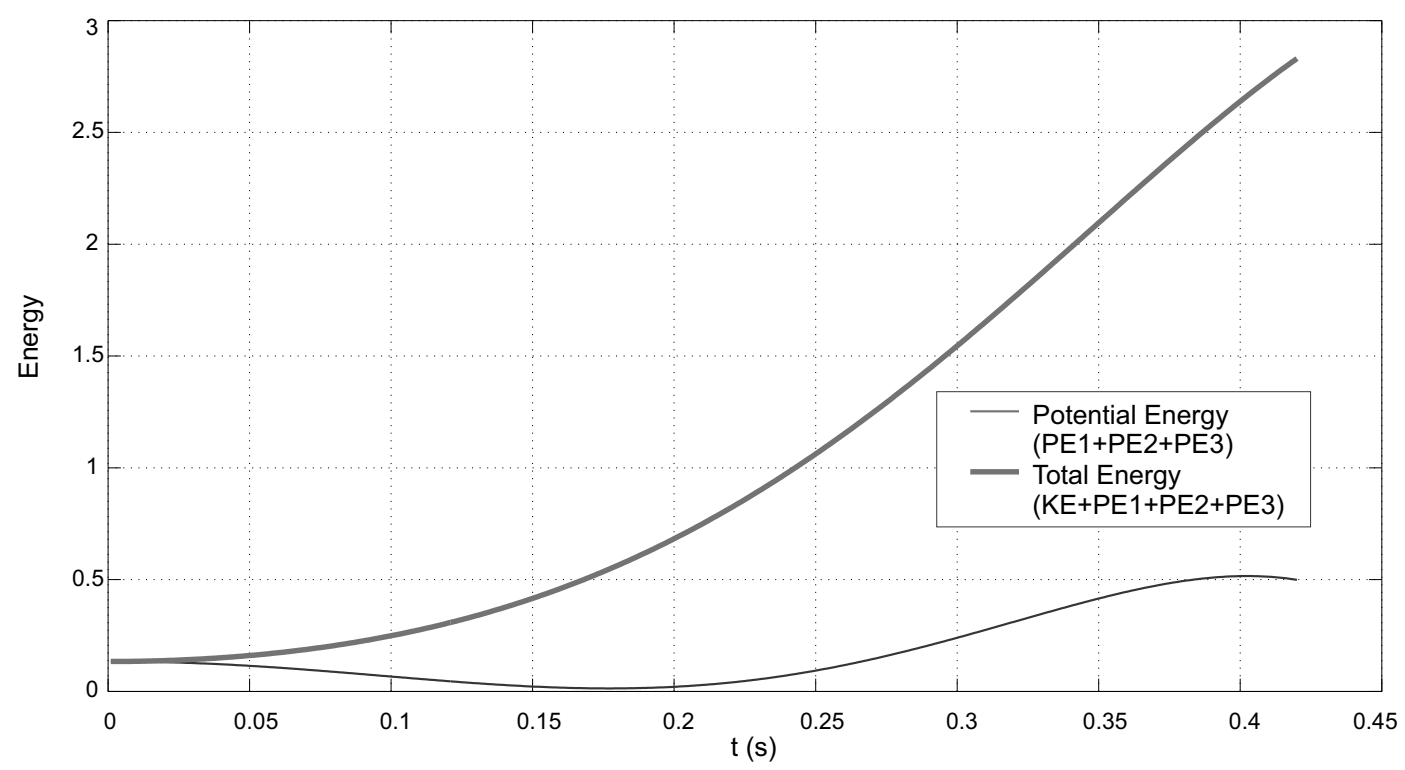

Figure 3-4: Total and potential energy variation during a fault-on trajectory computed using the TEF

In Figure 3-4, the potential energy curve represents the total of the 3 potential energy terms (PE1, PE2 and PE3) expressed in Equation (3.27). The maximum of the potential energy curve can be considered as $V_{c r}$ (Critical energy calculation methods are described in detail in Section 3.5). The energy acquired by the system at a particular time can be obtained by the total energy curve $(V)$. As per the step 6 
shown above, at a given time $t$ if $V<V_{c r}$, the postfault system is stable. Otherwise, the system may be unstable. Accordingly, Figure 3-4, if the value of the total energy curve at a particular time is less than the maximum of the potential energy curve, the the system is stable.

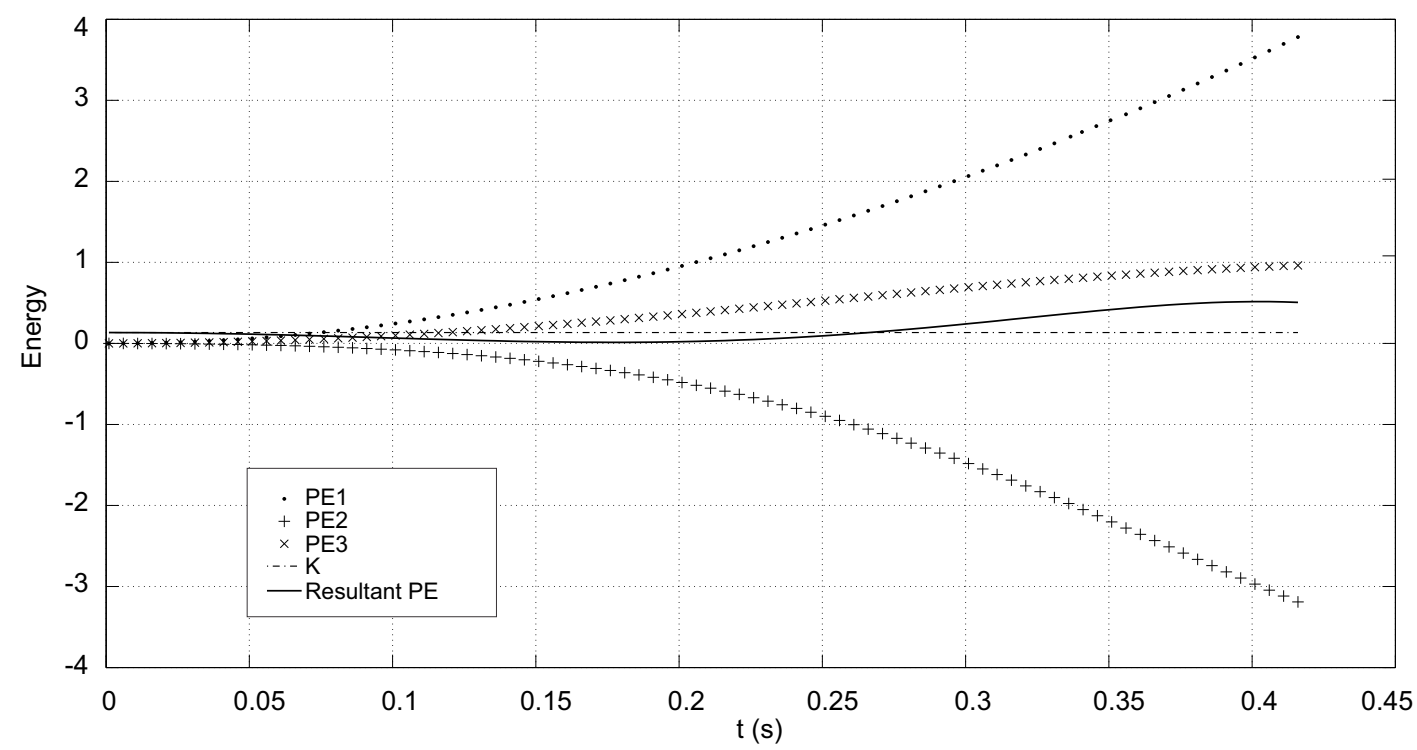

Figure 3-5: Contributions of the individual terms of the TEF for the potential energy

Figure 3-5 shows contributions of potential energy terms of the potential energy curve shown in Figure 3-4. The term $K$, referred to as $V$ correction term [54], is the potential energy difference of prefault SEP and the postfault SEP. If the fault is a self clearing fault, there will be no potential energy difference as both prefault and postfault systems have the same SEP, i.e. $K=0$. Otherwise, the potential energy difference is accounted in the TEF. Accordingly, $K$ will be a contingency dependant constant. Inclusion of potential energy difference of prefault SEP and the postfault SEP is explained in Section 3.4.2.

\subsubsection{Calculation of TEF Terms}

The TEF is used to calculate the energy acquired by the generators at a given time. The use of the TEF derived in 3.27 requires the computation of four terms $(K E$, 
PE1, PE2 and PE3). Computation of these terms requires a) the rotor angles and speed deviations of generators; and b) the postfault SEP. However, the term PE3 comprised of an integral term which is to be computed using numerical integration along the system trajectory. Several methods that can be used to compute this term are discussed in [52] where the path dependant integration using trapezoidal method is proposed as the most appropriate and a theoretically sound method. The application of the trapezoidal integration for computation of the term $P E 3$ leads to Equation (3.28). The value at simulation time step $n$ is obtained as follows:

$$
\begin{aligned}
I_{i j}(n) & =I_{i j}(n-1)+\frac{1}{2} D_{i j}\left[\cos \left(\theta_{i}(n)-\theta_{j}(n)\right)+\cos \left(\theta_{i}(n-1)-\theta_{j}(n-1)\right)\right] \\
& \times\left[\theta_{i}(n)+\theta_{j}(n)-\theta_{i}(n-1)-\theta_{j}(n-1)\right]
\end{aligned}
$$

Further, the initial condition for the system trajectories is the prefault SEP. Initial condition for the TEF is the postfault SEP. The potential energy at the postfault SEP is 0. If the contingency results in no network configuration change, there is no potential energy difference. Otherwise, there is a potential energy difference between two operating points and this has to be considered during the energy computation. In Figure 3-5, term $K$ shows the potential energy difference between the prefault SEP and the postfault SEP due to that particular network structure change. This is calculated by using potential energy terms (PE1, PE2 and PE3) of the TEF (Equation (3.27)) as shown in the following Equation (3.29).

$$
\begin{aligned}
V_{P E}\left(\theta^{0}\right)= & -\sum_{i=1}^{m} P_{i}\left(\theta_{i}^{0}-\theta_{i}^{s}\right)-\sum_{i=1}^{m-1} \sum_{j=i+1}^{m}\left[C_{i j}\left(\cos \theta_{i j}^{0}-\cos \theta_{i j}^{s}\right)\right] \\
& +\sum_{i=1}^{m-1} \sum_{j=i+1}^{m}\left[\int_{\theta_{i}^{s}+\theta_{j}^{s}}^{\theta_{i}^{0}+\theta_{j}^{0}} D_{i j} \cos \theta_{i j} d\left(\theta_{i}+\theta_{j}\right)\right]
\end{aligned}
$$

Assuming the prefault SEP and the postfault SEP are close, linear approximation 
can be used to compute the path dependant term. Accordingly, the TEF with the potential energy correction term is shown in Equation (3.30).

$$
V(\theta, \tilde{\omega})=V_{K E}(\tilde{\omega})+V_{P E}(\theta)-V_{P E}\left(\theta^{0}\right)
$$

As the initial difference of potential energy is considered in Equation (3.30), the initial value of the integration in Equation (3.28) is zero $\left(I_{i j}(0)=0\right)$.

\subsection{Methods for Critical Energy Calculation}

The accuracy of the critical energy, $V_{C R}$, estimation has a significant impact on the overall accuracy of the direct method of stability assessment. If the critical energy is calculated in a conservative manner, the results of the respective method becomes conservative. On the other hand, if the critical energy is an over estimation, the respective method will produce incorrect results.

In literature, researchers have obtained $V_{C R}$ by computing the potential energy at following four locations 1) Potential Energy Boundary Surface (PEBS) crossing point 2) Closest Unstable Equilibrium Point (UEP) 3) Controlling Unstable Equilibrium Point (CUEP); and 4) Boundary of Stability Region based Controlling Unstable Equilibrium Point (BCU).

The discussion on the stability region of an SEP in Section 3.1.5 can be extended to identify the above mentioned points. A power system, in its steady state, operates at the SEP. During a disturbance (e.g.: a fault), the system operating point moves away from the SEP. Assume that a fault is cleared without changing the network structure, the system operating point, according to Figure 3-3, can be a) within the stability region $b$ ) on the stability boundary; or $c$ ) outside the stability boundary. If the system operating point at the fault clearing time lies within the stability region (i.e.: a), the system will eventually approach the SEP, and the system state is classified as stable. If the system operating point lies outside the stability region (i.e.:b), the 
system state is classified as unstable. Assume if the system operating point at the fault clearing time lies on the stability boundary (i.e.:c), theoretically, the system trajectory evolves on the stability boundary and eventually converges to a UEP. The exact $V_{C R}$ is the potential energy computed at that particular UEP which is called the CUEP.

Among all the UEPs on the stability boundary, closest UEP is the UEP which has the lowest potential energy value for the energy function that is in use for stability assessment. The closest UEP may not be the controlling UEP [9]. PEBS is formed by joining the potential energy maxima of each UEP to form an equipotential boundary. For the SMIB system, the UEP can be analytically calculated. Furthermore, along the direction orthogonal to the PEBS, the potential energy $V_{P}$ achieves a local maximum at the PEBS [9].

The calculation of the CUEP for the SMIB system is analytical and straightforward (Appendix B). However, for a multi-machine power system with lossy network models, the CUEP is one of the UEPs in a large degree state-space. Therefore, the computation of the CUEP is highly numerically intensive [9].

\subsubsection{Potential Energy Boundary Surface (PEBS) Method}

The PEBS method was initially proposed in [14] and [51]. This method avoids the computation of the CUEP and requires only the faulted trajectory to be simulated. Further, the calculation of the postfault SEP can also be avoided depending on how far apart are the postfault and the prefault SEP. However, the PEBS method is incapable of consistently assuring the conservativeness of the stability assessment results [55]. It may produce overestimated or underestimated stability assessment results $[9,55]$. In literature, there are several modifications proposed to the PEBS method to improve the assessment accuracy.

The path dependent term in Equation (3.27) is obtained using the trapezoidal integration [52]. The faulted trajectory is simulated until the zero crossing point 
of Equation (3.31) is found, where $f(\theta)$ is the accelerating power of the post fault system, $\theta$ is the rotor angle at the current time step, and $\theta^{s}$ is the respective angle at the postfault SEP.

$$
f^{T}\left(\theta-\theta^{s}\right)=0
$$

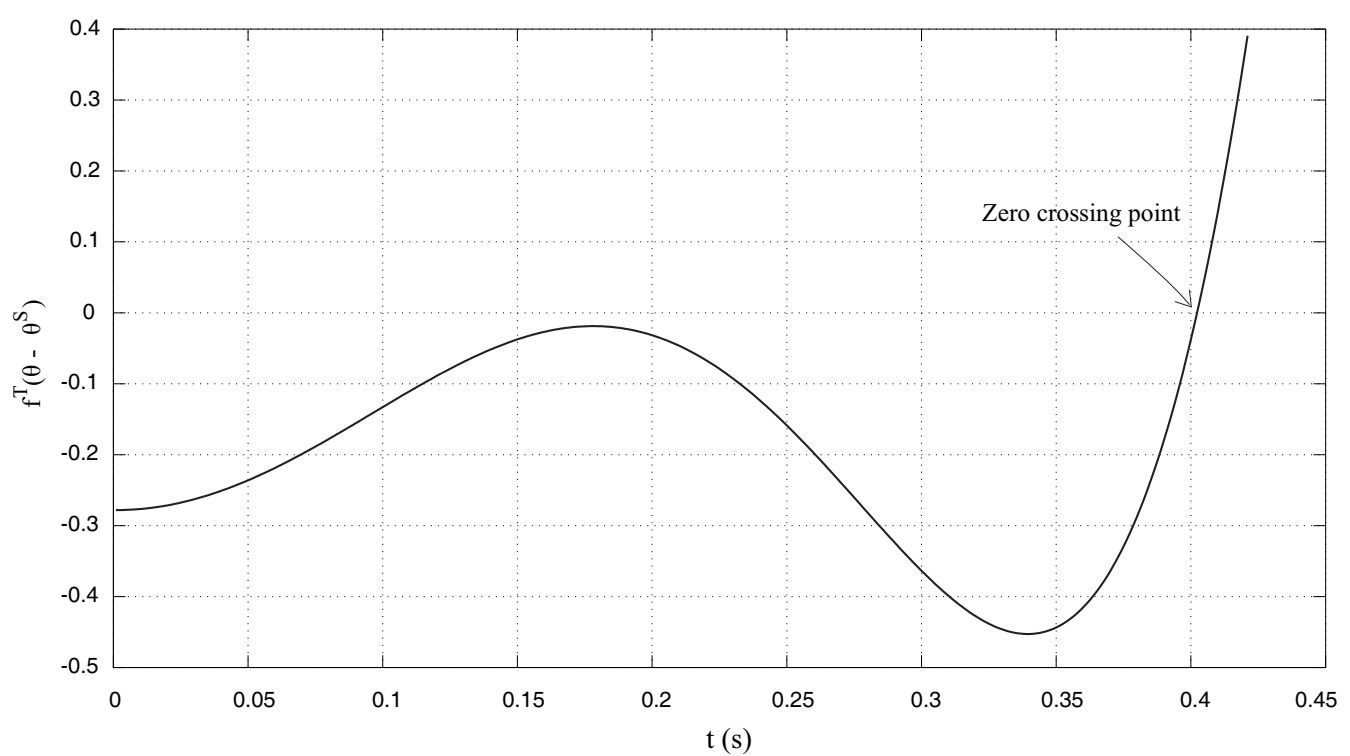

Figure 3-6: PEBS Crossing is identified at the zero crossing point of $f^{T}\left(\theta-\theta^{S}\right)$

Critical energy, $V_{C R}$, is calculated at the zero crossing point of the Equation (3.31). Figure 3-6 shows the Matlab simulation results of IEEE 3 generator 9 bus system to obtain the zero crossing point of the following contingency: three phase to ground fault occurred on line $5-7$ closer to the bus 7 and cleared by isolating the line $5-7$. A comparison of stability assessment accuracy of the PEBS method and the time domain simulations are presented in [56].

\subsubsection{CUEP Method}

The accuracy of the stability assessment of the PEBS method is enhanced if the $V_{C R}$ is calculated at the exact relevant CUEP. There are several methods proposed 
in literature to calculate the CUEP $[9,52]$. The method mentioned in [52] is to continue the integration of the reduced set of gradient Equation (3.32), by using the zero crossing point found in section 3.5.1 as the initial condition, until the minimum gradient point, which is the minimum of $\|f(\theta)\|$, is found. The rotor angles obtained at the $\|f(\theta)\|$ minimum are used to calculate the critical energy [52].

$$
\dot{\theta}=P_{m i}-P_{e i}-\frac{M_{i}}{M_{T}} P_{C O I}=f_{i}(\theta) \quad i=1, \ldots, m
$$

The time domain method to find the CUEP as mentioned in [9] is a three step procedure: 1) Iteratively compute the exit point relative to a fault-on trajectory; 2) Using the exit point as the initial condition, integrate post fault system until the sum of squares of speed state-variables is smaller than a threshold value; and 3) Solve the non-linear algebraic equations using the rotor angles found at the previous step as the initial condition.

If the minimum gradient point found in the former method or the point found at step 2 of the latter method is sufficiently close to the exact CUEP, solving the non-linear algebraic equations, $f(\theta)=0$, using an iterative solving technique such as the Newton-Rapson method would converge to the exact CUEP. However, the size of the region of convergence depends on the technique in use for solving the non-linear algebraic equations. Finding initial conditions within the convergence region of the CUEP is challenging for large systems with multi dimensional state space [9].

Figure 3-7 shows a phase plot of two rotor angles of IEEE 3 generator 9 bus test system during the CUEP computation process using the time domain method. The third generator is modelled as an infinite bus, therefore, no variation of rotor angle is observed. The CUEP is calculated relevant to a three phase to ground fault occurred on line $5-7$ closer to the bus 7 , and cleared by isolating the line 5-7.

Initially, the system is at the prefault SEP. The value of two rotor angles are indicated by point $a$. The post fault SEP relevant to the contingency considered here is also shown in Figure 3-7. The exit point, $b$, is assumed to be on the stability 


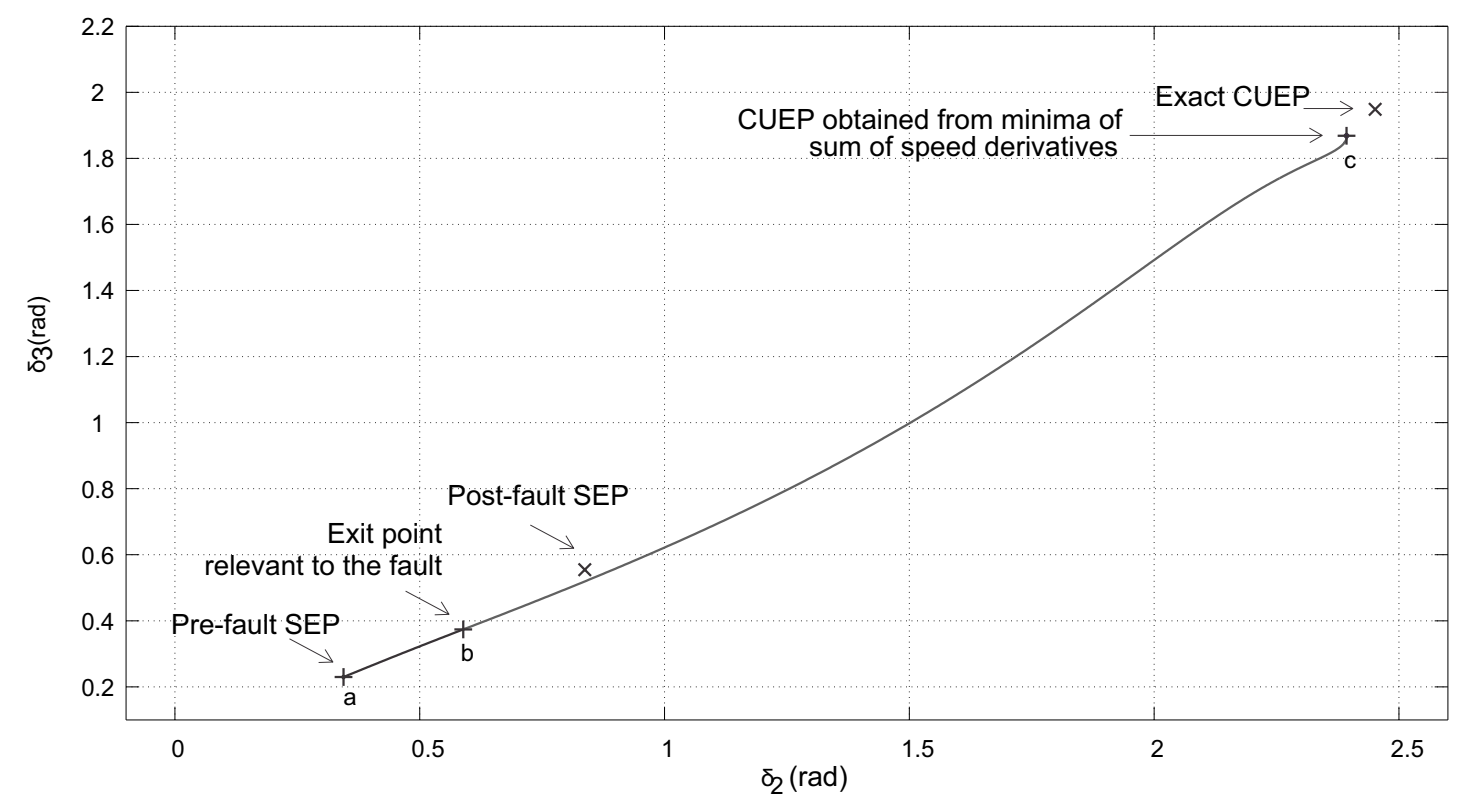

Figure 3-7: Phase plot of the CUEP computation using the time domain method

boundary. In order to find the exit point, a three phase to ground fault is applied and cleared by isolating the line 5-7 (similar to the contingency). If the system converges to the postfault SEP, then the fault duration is increased step by step until the postfault system diverges. Otherwise, the fault duration is decreased step by step until the postfault system converges to the SEP. This iterative process identifies two consecutive time steps where the exit point of the system trajectory lies somewhere in between. Bisection rule is proposed in [9] to be applied in between two time steps to further enhance the accuracy of the exit point calculation.

When the fault is cleared exactly on the exit point, the system trajectory lies on the stability boundary of the postfault system. According to the stability theory [9], the system trajectory evolves on the stability boundary and eventually converges to a CUEP on the stability boundary, which is the controlling CUEP. Accordingly, the postfault system is simulated by using the previously determined exit point as the initial conditions. Due to the numerical errors that occur in the simulation, the system trajectory is unlikely to follow the stability boundary. Therefore, during the simulations, the total sum of square errors of speed trajectories was observed 
against a threshold value (theoretically the speed deviations become zero once the system trajectories converge to an UEP). Finally, as mentioned in step 3 of the time domain method to find the CUEP, the non-linear algebraic equations are solved using the initial conditions at point $c$ to find the exact CUEP. In this case, the NewtonRaphson algorithm converged to the exact CUEP. Even if it is not converged to the exact point, usually, point $c$ is a sufficiently close point to the exact CUEP.

CUEP method is recognized as the most viable method for direct stability assessment of power systems.

\subsubsection{Closest UEP Method}

The closest UEP is defined as the UEP on the stability boundary that has the lowest potential energy among all the UEPs on the stability boundary [57]. This method does not consider the relevant CUEP, therefore, it does not consistently provide an accurate approximation of the relevant stability boundary [58]. Figure 3-8 shows the simulation results of the potential energy variation for an SMIB system. The SEP and the two UEPs are shown the figure. In this specific situation the CUEP and the closest UEP refers to the same UEP.

\subsubsection{Boundary of Stability Region based Controlling Unsta- ble Equilibrium Point (BCU) Method}

Although the CUEP method is considered as the most viable direct method for power system applications, the task of computing the exact and the relevant CUEP to a contingency is a difficult task.

The BCU method proposes to develop the models tailored to the underline power system transient stability model along with mathematical insights. This model is an artificial, reduced-state model capturing all the equilibrium points in the power system model under investigation. 


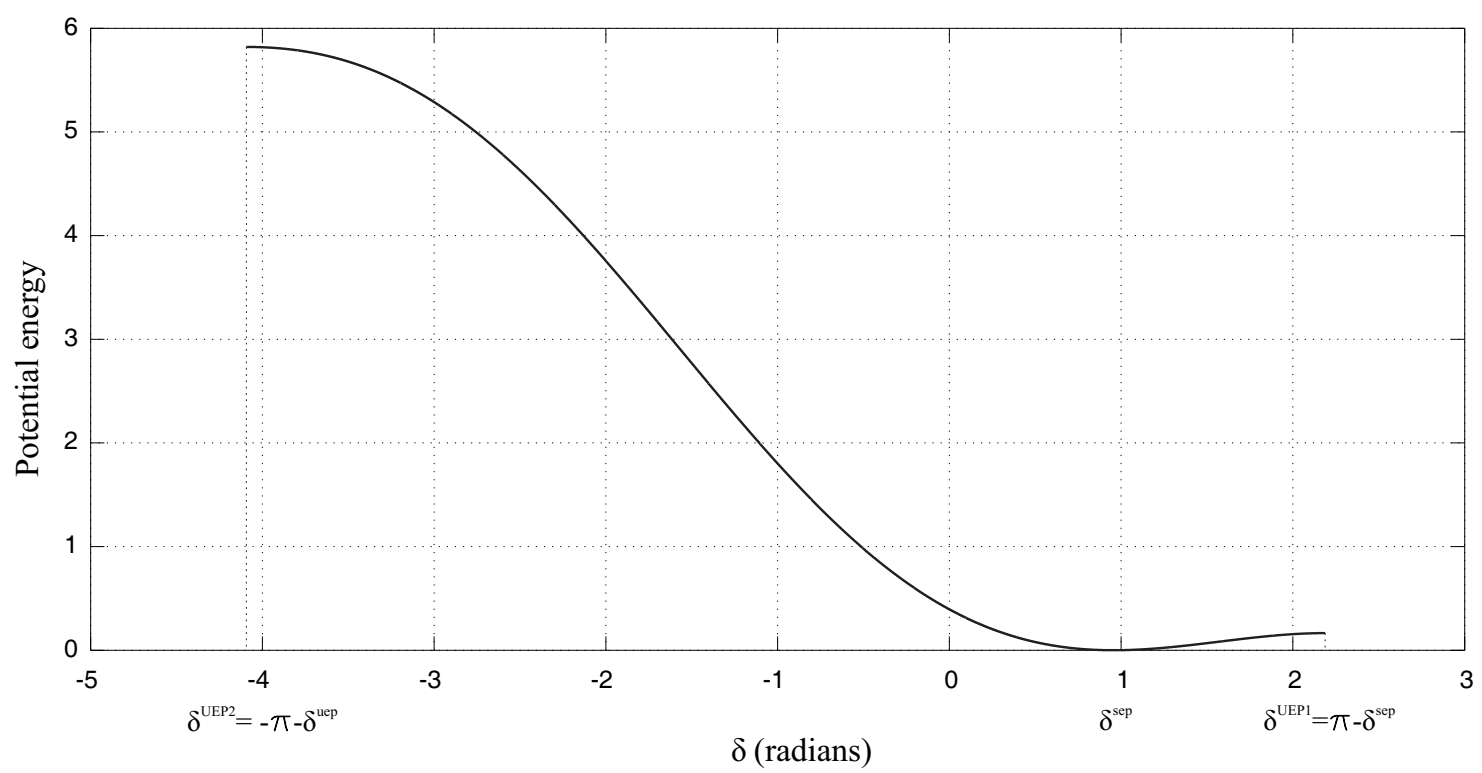

Figure 3-8: Potential energy vs rotor angle, including two UEPs and the SEP for a SMIB system

The authors of [9] propose following two sets of properties associated with the original model to be satisfied with the reduced-state model.

\section{Static Properties}

- equilibrium points of the reduced-state model should have the corresponding equilibrium point in the original system model.

- type of the equilibrium point of the reduced-state model should be the same type of an equilibrium point of the original system model.

\section{Dynamic Properties}

- A valid CLF should exist for the reduced-state model.

- An equilibrium point on the stability boundary of the reduced-state model should be on the stability boundary of the original system model.

- It should be computationally feasible to efficiently identify the intersection of the fault-on trajectory and the stability boundary of the reduced-state system 
without performing a detailed time domain simulation.

The CUEP of the original model is computed by computing the CUEP of the reducedstate model of which the CUEP can be effectively computed due to the smaller number of states.

\subsubsection{Other Attempts}

This section provides a brief background to a set of selected energy functions proposed in the literature for the completeness. All of these energy functions are numerical energy functions. The main objective of proposing various energy functions is to improve the accuracy of stability assessment by incorporating detailed device properties and to include different power system devices (such as High Voltage Direct Current (HVDC) and FACTS devices.

There are many attempts reported on incorporating detailed device models into energy functions. A 23 term energy function comprised of 13 path dependant numerical terms has been proposed in [9]. This energy function considers the rotor circuit equations of the two-axis detailed generator model. An energy function for a detailed generator model with a simple exciter (one gain and one time constant) is presented in [59]. Incorporating non-linear load models [60,61] and FACTS devices (Section 5.4.8) has been also discussed in literature. The effect of load models has been analysed to improve the accuracy of the first swing TSA.

Initially, the energy functions have been developed for loss-less network-reduction models. In loss-less power system models, the transfer conductances in the Y matrix are ignored. This representation includes the loads in the Y matrix by computing respective constant admittances [48]. The network is then reduced to the generator internal buses. Although no analytical energy functions exist for lossy multi-machine power system models, lately, network preserving numerical energy functions have been proposed with an aim to incorporate the transfer conductances in the energy functions and for a better representation of the transmission network instead of network 
reduction [60-62]. These methods were not attractive due to the level of complexity and the topological dependence $[9,59]$.

The use of energy functions in this thesis is limited to computing a set of input features for machine learning. The transient stability of the system is estimated not only based on the TEF outcomes. Since the potential application of the proposed technique is the on-line DSA, the processing power requirement is a main factor to determine the feasibility of application. The TEF based pre-processing is designed to have a balance between the complexity of processing and the effectiveness of pre-processing. Therefore, the direct method based techniques and energy functions involving highly numerically extensive computations are not discussed in detail in this thesis. Further, BCU methods requires the validity assessment of the reduced state model against the original system model. This process requires the examination of certain properties of both system models which requires the physical and mathematical insights of the power system transient stability model. The applicability of these methods in on-line DSA were not comprehensively studied and reported in literature.

\subsection{Limitations and Issues of Direct Method of Stability Assessment}

Direct method has the advantage of stability assessment without performing the time domain numerical integration of the postfault system, i.e., time domain simulation is required only upto the fault clearing time. However, there are several limitations and issues for direct methods to overcome before becoming a practical tool for power system transient stability analysis.

Following are a list of issues and limitations applied to direct methods:

- the energy functions are derived in a way assuming the postfault system should be autonomous as shown in Equation (3.2). Therefore, the sequence of events has to be defined in advance. 
- the existence of an energy function is not guaranteed for every power system model.

- no analytical energy functions exist for lossy systems.

- challenges in computing the exact CUEP; and

- mostly applicable for the first swing stability analysis $[9,48]$.

\subsection{Concluding Remarks}

This chapter presented a brief introduction to non-linear systems theory in relation to the direct method of stability assessment. The application of the direct method of stability assessment to power systems was discussed. The derivation of the TEF and the numerical energy functions were presented. Several critical energy computation algorithms were discussed and illustrated using numerical simulations. 


\section{Chapter 4}

\section{Automated Database Generation for Dynamic Security Assessment}

\subsection{Introduction}

The development of the data generation algorithms for machine learning based applications require a significant portion of time of a research or a study. Therefore, having a ready-made and a customizable tool is an advantage. This chapter presents the design and the development details of automated database generation algorithms developed during the research reported in this thesis. The developed software is not system specific and it is a stand-alone tool for data generation for power system stability studies. Further, it provides means to incorporate system specific constraints and corrective actions based on the insights of the system operation engineers. The developed software has been configured to optionally compute the potential and kinetic energy terms based on the TEF method at a given time during the transient stability simulations. These datasets can be exported conveniently for supervisory learning applications. The software comprised of a web-based interface that allows the authenticated users to operate remotely without explicit software installations on the client computer. 


\subsection{Requirement Specifications}

Supervisory learning applications require datasets generated for training, testing and validation of learned networks. The quality of these datasets determine the prediction accuracy of the learned network. The speed of the data generation process plays a key role when the application is for time critical reporting such as real-time DSA of electrical power systems. Further, the complexity of the power system model makes the data generation process computationally demanding.

A data generation software system for application of supervisory learning for power system on-line DSA needs to address the following key aspects

1. Generate representative datasets for training, testing and validation.

2. Fast generation of datasets to enable application in on-line DSA.

3. Ability to integrate with the other functional units of the on-line DSA implementation.

Accordingly, the automated data generation software system, Machine Learning for Dynamic Security Assessment (ML4DSA), has been developed to fulfil the following list of functional and non-functional requirements:

\section{Functional requirements.}

The ability to:

1. specify the range of the operating point deviation to be accommodated in a particular dataset.

2. incorporate the user defined corrective actions

3. classify a given operating point as acceptable or unacceptable depending on a set of user defined constraints.

4. use the existing power system models, obtain the power flow and the dynamic simulation results using the industry accepted tools. 
5. continuously generate the required number of acceptable steady state operating points and then perform the dynamic simulations for each contingency in the set of credible contingencies; and

6. to conclude the stability status of a particular dynamic simulation according to a given stability assessment algorithm.

\section{Non-functional requirements.}

The ability to:

1. specify various parameters required for the process using a convenient user interface.

2. archive simulation results and datasets and serve for extended applications and reporting; and

3. specify access rights to operators performing different roles.

The system is designed in a task oriented modular manner. Tasks can be identified into the following main categories: data input, perturbation and random operating point generation, case specific corrective actions, dynamic simulation and stability assessment.

\subsection{System Structure}

Python algorithms of ML4DSA execute on the Web2Py ${ }^{\text {TM }}$ Web Framework (Web2Py) framework [63]. As shown in Figure 4-1, ML4DSA interacts with Power System Simulator for Engineering ${ }^{\circledR}$ (PSSE) using the Psspy module [64] available in PSSE. The automation process creates two sets of files using PSSE: steady state operating points (SAV files) and time series data of dynamic simulations (OUT files). Time series data made available to ML4DSA using the Dyntools [64] module available in PSSE 


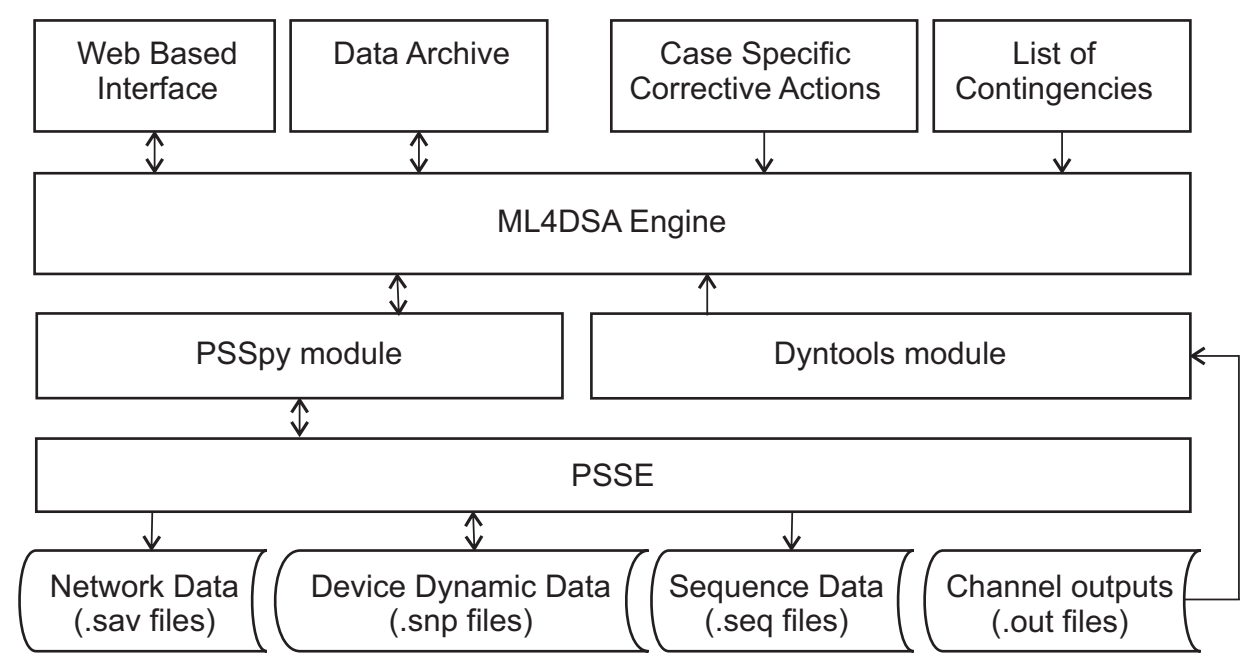

Figure 4-1: Overall block diagram of ML4DSA.

\subsubsection{Inputs}

ML4DSA requires PSSE to be installed on the server computer. Initially, it locates the PSSE installation and establishes the connection by starting a PSSE instance. Power system network structure is introduced to ML4DSA by using the standard SAV type file. As with the other input files to the system, SAV files are stored in the database to enable the user to have easy access to input data within ML4DSA. In addition, dynamic simulations require the device model parameters of the dynamic devices in the power system. In addition, unbalanced network simulations require the sequence data. Typical dynamic devices are the electrical machines together with their auxiliary controls (such as exciters, governors, stabilizers, limiters, etc.), FACTS devices and loads. The dynamic device models are introduced to ML4DSA using the standard SNP type file. ML4DSA is capable of reading the contingency specification files. Also, the user can construct the list of credible contingencies according to the format compatible to ML4DSA's contingency reader. 


\subsection{Steady State Operating Point Generation}

The electrical power generation levels and the unit dispatch changes continuously with varying loads and system topology to maintain the power system in normal operation. Load forecasting provides accurate means to predict load changes for a period of time ahead. To produce a learned network using supervisory learning, it is necessary to have the knowledge of the range of variation of the system parameters to generate the database representing the entire range of parameter deviation. ML4DSA allows the user to specify the range of parameter deviation for each generator and load in the power system. Active and reactive power of the loads have been varied respectively by using (4.1) and (4.2) [65]. $\varepsilon$ is a uniform independent random variable between 0 and 1 . For example, $\pm 20 \%$ deviation for active and reactive power of the load on bus $i$ is enabled by setting $\Delta P^{(i)}=\Delta Q^{(i)}=0.2$.

$$
\begin{aligned}
& P_{L}^{(i)}(k)=P_{L O}^{(i)}\left\{1+2 \Delta P^{(i)}\left[0.5-\varepsilon_{P L}^{(i)}(k)\right]\right\} \\
& Q_{L}^{(i)}(k)=Q_{L O}^{(i)}\left\{1+2 \Delta Q^{(i)}\left[0.5-\varepsilon_{Q L}^{(i)}(k)\right]\right\}
\end{aligned}
$$

Active power generation and the terminal voltage of a generator bus were varied respectively using (4.3) and (4.4). Accordingly, $\pm 20 \%$ change of the power reference and the terminal voltage by $\pm 2 \%$ of the base case conditions is allowed by setting $\Delta P^{(i)}=0.2$ and $\Delta V^{(i)}=0.02$ respectively.

$$
\begin{aligned}
P_{G}^{(i)}(k) & =P_{G O}^{(i)}\left\{1+2 \Delta P^{(i)}\left[0.5-\varepsilon_{P G}^{(i)}(k)\right]\right\} \\
V_{G}^{(i)}(k) & =V_{G O}^{(i)}\left\{1+2 \Delta V^{(i)}\left[0.5-\varepsilon_{V G}^{(i)}(k)\right]\right\}
\end{aligned}
$$

Then, the power flow is solved with the changed loading and generation. IEEE 39 bus 10 generator power system model has been used for the data generation reported 
in this chapter. The power mismatch during network structure changes is assumed to be supplied by the swing generator. If the power flow is converged, the general limit checks are performed on the new operating point to identify if that is an acceptable operating point. If the violations are identified, the particular operating point is discarded. If not, that operating point is saved into the database.

For the convenience of operation rather than individual parameter specification, the user can specify a maximum deviation to generate random deviations for all loads and generators. Perturbed values for loads and generation are stored in the database and are editable by the user. If the user wants to keep some loads or generators unperturbed over the database generation process that is allowed by setting the deviation to zero in the perturbation parameters for that particular device(s) in the database. ML4DSA is capable of iteratively generating a user specified number of acceptable operating points. Maximum number of iterations is specified as a parameter to avoid indefinite looping if no acceptable operating points exist. The process flow chart to create a single operating point by perturbing the load and the generation is shown in Figure 4-2.

The process begins by solving the power flow using PSSE for perturbed loads and generation to check if there is a steady state operating point. If there is no power flow solution, ML4DSA ignores the set of randomly perturbed loads and generation and starts from the beginning. If the power flow is converged, the algorithm proceeds to system specific limit checks to decide if the operating point is an acceptable operating point. A screenshot of the ML4DSA interface for operating point generation is shown in Figure 4-3. 


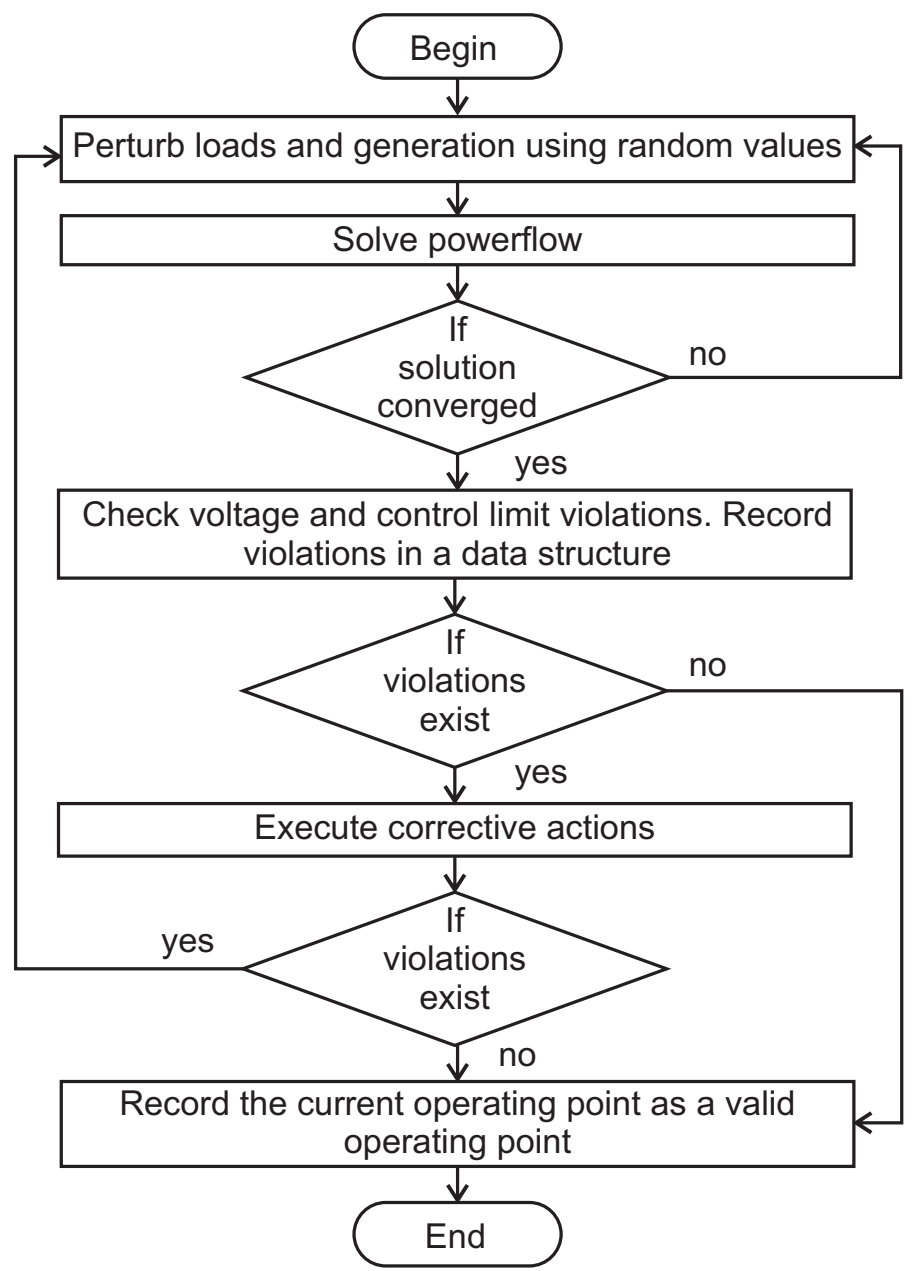

Figure 4-2: Overall block diagram of acceptable steady state operating point generation process. 


\section{ML4DSA}

Power systems Research Group | University of Manitoba

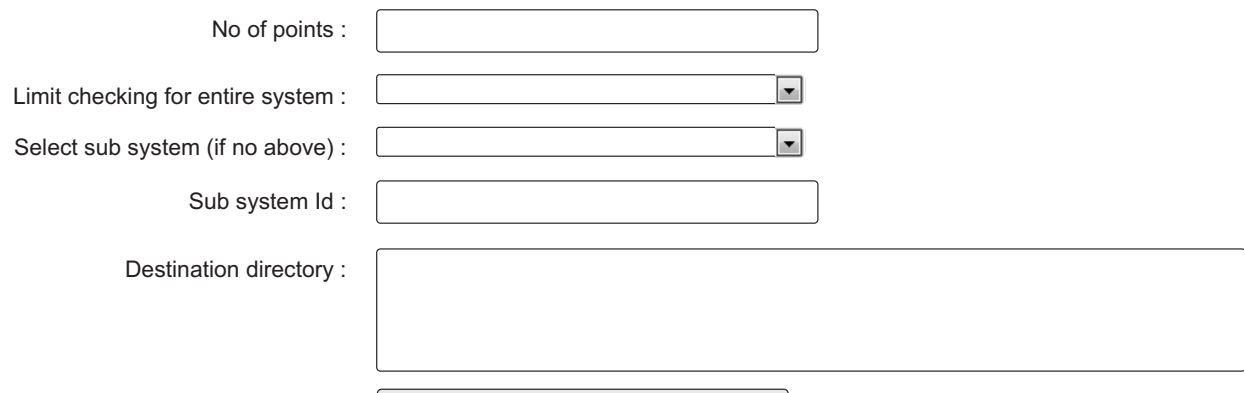

Generate Secured Operating Points

Figure 4-3: A screen-shot of the ML4DSA interface for acceptable operating point generation.

\subsection{System Specific Limit Checks and Corrective Actions}

The power system behaviour varies from system to system. The knowledge and insight gained by various studies and actual system events help the operators to allow certain violations acceptable to a particular system. For example, the reliability criteria imposed on systems sometimes require a part of the system to operate strictly within tight voltage limits when compared to the rest of the system. Therefore, the data generation program should accommodate the system specific operating conditions.

ML4DSA facilitates packaging the system specific rules and parameters in a single folder as shown in Figure 4-4. Rules are scripts written in Python and parameters are in the form of comma separated text files. For example, to allow a higher voltage limit in a particular bus, the user can write a Python script in the rule set folder accessing the bus number and the new limits in the respective text file in the parameters folder. Having the parameters separately from rules allow the reuse of rules and alternation 


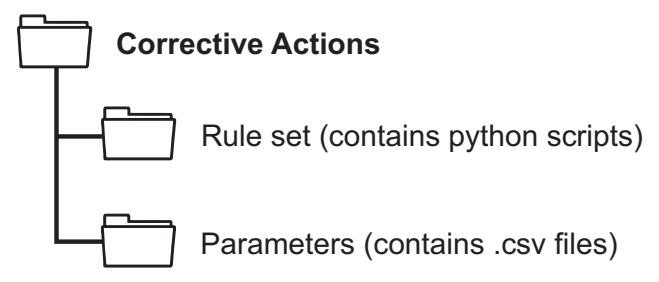

Figure 4-4: Structure of a typical corrective actions folder compatible for ML4DSA.

of parameters without making changes to Python scripts. Data interchange between ML4DSA and Python scripts for specific corrective actions is performed via two shared data structures.

The corrective actions folder is read by ML4DSA during the random operating point generation. The user can have different folders containing system specific corrective actions and parameters for different studies. Further, ML4DSA allows the rule selection to enable or disable individual rules in a set of rules.

\subsection{Automated Dynamic Simulations}

Once the acceptable steady state operating points are created, for each operating point, the system stability under each contingency is assessed. This involves the time domain simulation of each contingency and creating the time series data (OUT files). ML4DSA creates a separate folder for each perturbed operating point and stores its contingency simulations outputs within. The process flow chart for dynamic simulation is shown in Figure 4-5. The naming convention for output files (OUT files) is the contingency number followed by the contingency name.

The set of contingencies to be applied are input to the system using a text file. ML4DSA is also capable of reading the contingency specification file format used in Transient Security Analysis Tool ${ }^{\circledR}$ (TSAT). Preparation of the list of contingencies in the above mentioned format is straightforward. ML4DSA has the capability of generating the respective PSSE commands required to perform dynamic simulation 
for the set of input contingencies. Generated PSSE commands are stored in the database and sequentially executed during dynamic simulation of contingencies.

By default, PSSE records a large number of output channels during dynamic simulations which involves a significant overhead time for text file manipulation. ML4DSA allows the user to specify the necessary output channels to be recorded. Recording only the necessary channels enhance the simulation efficiency as well as enables faster reading of OUT files by the stability assessment algorithms.

\subsubsection{Stability Classification}

ML4DSA facilitates integration of different stability assessment algorithms. Further, it is also capable of reading the simulation output channels, created during the dynamic simulations, to the Python environment as per the requirement of the stability assessment algorithm.

At the present stage of development, ML4DSA is capable of assessing the system stability using the power angle based stability margin [40]. This index is being used only to classify stable and unstable operating points under the list of credible contingencies. The assessment results are presented to the user using a boolean value as shown in the Figure 4-6. The simulation id is a unique alpha numeric value that distinguish a database generation cycle. Moreover, the simulation $i d$ allows the user to record and retrieve results of every database generation attempt.

\subsubsection{TEF Terms Computation}

A general list of steps for stability assessment using the TEF method is listed in 3.4.1. In this thesis, the computation results of the TEF method is not directly used to assess the stability. Corresponding value of each term of the TEF (KE, PE1, PE2, and PE3) for the set of generators are computed for each operating point under each credible contingency. This requires one additional dynamic simulation of the fault-on system as mentioned in Section 4.6. The simulation time of this simulation is usually 


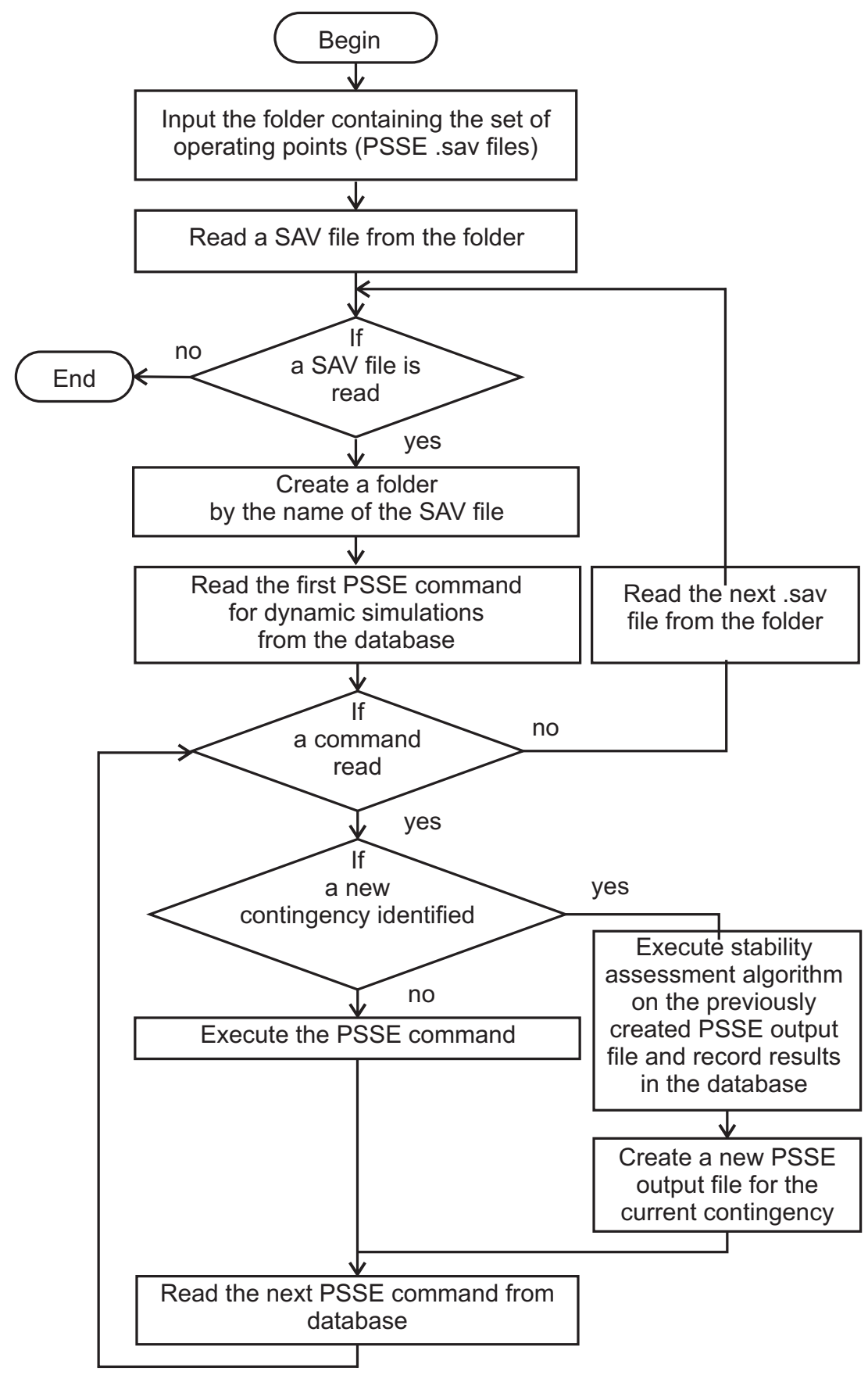

Figure 4-5: Process flow of iterative dynamic simulation. 


\section{Stability Assessment}

\begin{tabular}{|c|c|c|c|c|c|}
\hline Query & & & Search & \multirow{2}{*}{\multicolumn{2}{|c|}{162 records found }} \\
\hline Add & Export & & & & \\
\hline Id & Simulation Id & Sav file & Contingency & Stability & \\
\hline 1 & test & perturbed_1.sav & 1_Bus_12 & 1 & View \\
\hline 2 & test & perturbed_2.sav & 1_Bus_12_u & 0 & View \\
\hline 3 & test & perturbed_3.sav & 1_Bus_12 & 1 & View \\
\hline 4 & test & perturbed_4.sav & 1_Bus_12_u & 0 & View \\
\hline 5 & test & perturbed_5.sav & 1_Bus_12 & 1 & View \\
\hline
\end{tabular}

Figure 4-6: Screen-shot of stability assessment of a dynamic simulation.

about 5 to 15 cycles $(83-250 \mathrm{~ms})$. The computation method has been described in detail in Section 3.4.2.

\subsection{Testing Process}

The capability of the developed software system has been tested for the computational accuracy and for the computation speed for two power system models.

1. New England 39-bus test power system model; and

2. MRO power system model with over 50,000 buses.

The steady state operating point generation in Section 4.4 and application of case specific limit checks and corrective actions in Section 4.5 have been tested using both power system models. Automated dynamic simulations in Section 4.6, the stability assessment based on the angle margin algorithm in Section 4.6.1 and the TEF term computation in Section 4.6.2 have been tested using the IEEE 39 bus 10 generator test power system model. 
The bottom-up testing and integration testing strategies have been used during the software development.

\subsection{Additional Features of the Software}

\subsubsection{Web Interface}

ML4DSA comprised of a web-based interface. The application is developed on Web2Py which is a free and an open-source web framework for agile development of secure database-driven web applications. Web2Py facilitates the software design using the Model View Controller (MVC) pattern by separating the data representation (the model) from the data presentation (the view) [66]. Moreover, the framework facilitates the use of Python language throughout the different stages of development.

ML4DSA also facilitates the user authentication by utilising the features available in Web2Py. The user authentication allows the implementation of software access policies within an organization in order to ensure the fair access to propriety software resources.

\subsubsection{Configurability}

The developed software system is conveniently configurable to different types of:

- power system models,

- stability assessment algorithms; and

- contingencies including balanced and unbalanced fault conditions.

\subsubsection{Relational DBMS Integration and Study Reports}

ML4DSA is connected to a SQLite [67] relational Database Management System (DBMS). This database connectivity enables the ML4DSA to archive intermediate 
data computed during the computational process. For example, stability classifications, computed TEF terms, generator bus voltage magnitudes and phase angles are available to view as reports or to download as datasets. These data can be utilised by various extended applications.

\subsection{Concluding Remarks}

The steps involved in data generation for supervisory learning applications for power systems transient stability assessment were explained. The requirement specification and the development details of the stand-alone software system, ML4DSA, were presented. Practical implementations of on-line DSA systems, based on supervisory learning techniques, requires fast data generation algorithms with a possibility of integrating with the other functional units.

ML4DSA automates PSSE. This can also be used for automated data generation for power system studies in general. Further, it facilitates the user to plug-in the customized algorithms for case specific limit checks and for system specific corrective actions. The web-based software interface enables access over the web requiring

no additional software installed on client computers. Built in database integration provides convenient access to resources. 


\section{Chapter 5}

\section{Proposed Method for TSA using Energy Based Power System Features and Machine Learning}

\subsection{Introduction}

This chapter presents the algorithm proposed in this thesis to enable more frequent assessment cycles in on-line DSA, based on energy based power system features and machine learning. The assessment accuracy of the proposed method is examined in detail, for datasets of different sizes, operating conditions and disturbances. The inclusion of FACTS devices in the proposed algorithm is discussed. Further, the method of practical implementation using two simultaneously running processes is discussed. The computational resource requirements and the ways of achieving parallelism on computation is discussed. Finally, the advantages and the significance of the proposed method is discussed in relation to the similar methods proposed in recent literature. 


\subsection{The Concept of Combining Direct Methods and Machine Learning}

Power system rotor angle instability is a result of the synchronous machines being unable to release the energy accumulated during a disturbance to reach a new steady state. Accordingly, the energy accumulation of generating units are direct indicators of the stability of the system. Energy functions, that are the basis of stability assessment in direct methods, represent the energy acquired by the generators at a given time subsequent to a disturbance. Although the assessment accuracy of direct methods is insufficient for on-line DSA, the terms calculated by the energy functions can be considered as a set of pre-processed meaningful input features to a machine learning algorithm. The significance of the direct method of stability assessment is that the numerical integration is required only on the fault-on trajectory, usually less than $500 \mathrm{~ms}$, whereas the stability assessment using the time domain integration requires at least about $3 \mathrm{~s}$ of simulation time of the post fault system to conclude the stability status [8].

Recent literature on the application of machine learning for TSA have proposed to use the following raw network quantities as candidate input features: a) generator bus voltage magnitudes $[17,18,23]$; $b$ ) generator bus voltage phase angles $[15,18,19]$; and c) active and reactive power injections of individual generators [18]. These network quantities could be captured from the prefault system, at the fault clearing time or several cycles after the fault clearing time. When the energy terms calculated by the energy functions are used as input features, the input-output relationship to be captured by the supervisory learning technique is expected to be less complicated compared to the use of above mentioned raw network quantities as inputs. Therefore, it can be expected that the machine learning algorithm would be able to capture the underlying relationships with smaller sized training datasets and with higher accuracy when meaningful features are used as inputs. The reduction of the size of the required 
training dataset will in turn result in shorter data generation time enabling more frequent computational cycles.

Further, the thesis proposes to use a) load forecasting data; and b) a snapshot obtained from the EMS to estimate an appropriate range for the operating point deviation. A network trained using the data generated in this manner is used for the next computational cycle. Therefore, the trained network has the most recent information about the system topology and operating conditions. The proposed technique requires numerical integration of the fault-on system to calculate the energy terms. However, it is proposed to use simplified generator models (classical generator model) to perform the numerical simulation and to calculate the energy terms.

\subsection{Proposed Method}

\subsubsection{A Simple Example}

The following example is presented to demonstrate and investigate the performance of the learnability with two different input sets. The non-linear function shown in Equation (5.1) has inputs $a, b, c, d$ and $e$.

$$
y=a+b \sin (c)+c \cos (d)+e^{2} \sin (a)
$$

The variables $t_{1}, t_{2}$ and $t_{3}$ are obtained using inputs $a, b, c$ and $d$ as shown in equations (5.2), (5.3) and (5.4). $t_{1}, t_{2}$ and $t_{3}$ serve as a set of pre-processed inputs to the original function given in Equation (5.1).

$$
\begin{aligned}
& t_{1} \leftarrow a+b \sin (c) \\
& t_{2} \leftarrow \cos (d) \\
& t_{3} \leftarrow \sin (a)
\end{aligned}
$$


The simplified equation with the introduction of the pre-processed variables is given in Equation (5.5). In order to perform the pattern classification, output $y$ in equations (5.1) and (5.5) is converted to a boolean value by considering the condition $y \geq 0$.

$$
y=t_{1}+c t_{2}+e^{2} t_{3}
$$

The hypothesis is that if the machine learning technique is fed with a pre-processed set of data, the learning process becomes a) possible with a smaller dataset; $b$ ) more accurate; and $c$ ) faster. In this analogous example, two neural networks are trained to map the inputs and outputs of the non-linear functions given in equations (5.1) and (5.5). The input set for the first network is the randomly generated variables $a$, $b, c, d$ and $e$. The second network is trained using the pre-processed inputs $t_{1}, t_{2}$ and $t_{3}$ along with the raw inputs $c$ and $e$ as shown in equations (5.2), (5.3) and (5.4).

Table 5.1: Cross validated classification accuracy of two networks trained using raw and preprocessed inputs

\begin{tabular}{|l|l|l|l|l|}
\hline Dataset & $\begin{array}{l}\text { Dataset } \\
\text { size }\end{array}$ & $\begin{array}{l}\text { Number } \\
\text { of hidden } \\
\text { nodes }\end{array}$ & $\begin{array}{l}\text { Number of } \\
\text { inputs }\end{array}$ & $\begin{array}{l}\text { Accuracy of } \\
\text { classification } \\
(5-\text { fold CV })(\%)\end{array}$ \\
\hline raw & 200 & 14 & 5 & 83.0 \\
\cline { 1 - 1 } pre processed & 200 & 35 & \multirow{2}{*}{5} & 96.5 \\
\hline \hline raw & 100 & 14 & & 95.5 \\
\hline pre-processed & & & & 95 \\
\hline
\end{tabular}

Table 5.1 lists the specifications of the trained networks and the classification accuracy resulted in 5-fold cross validation. The first two rows indicate the classification accuracy of two networks trained using raw input features and pre-processed input features (i.e. dataset size, number of hidden nodes and number of inputs). It can be seen that the pre-processed input features are capable of producing more accurate estimations. The third row lists the classification accuracy of a network trained with the original dataset (i.e. 200) to obtain the best accuracy. In this case, the higher 
classification accuracy (93.5\%) is obtained by using a higher number of hidden nodes (i.e. 35). However, the best classification accuracy achieved was still lower than what was obtained using the pre-processed input features. Also, as listed in the fourth row, when trained with pre-processed input features, the accuracy drops only marginally (from $96.5 \%$ to $95 \%$ ) when the dataset size was reduced to a half (i.e. 100). Moreover, the classification accuracy was still higher than the best accuracy achieved using the raw input features.

The results indicate that a network trained with pre-processed data outperforms the network trained using the raw inputs in following ways:

1. smaller dataset is sufficient to produce a well trained network;

2. faster training process due to lesser hidden nodes used to capture the nonlinearities; and

3. higher prediction accuracy.

\subsubsection{Candidate Input Features of the TEF}

The example in Section 5.3.1 demonstrated the advantage of appropriately preprocessing the input features. The meaningful information captured in the TEF terms can be considered as an intermediate set of results of the stability assessment. Therefore, it is logical to consider the energy terms as a pre-processed set of input features. The use of said features as inputs to the machine learning application is expected to produce accurately classifying networks using smaller training datasets. This will also result in a shorter duration for data generation which will in turn enable more frequent computational cycles as well as accurate security assessments.

Classical energy functions are derived for the simplified power system model (clas-

sical generator model and the constant impedance load model) shown in Equation (3.24) [48]. Equation (3.7) has been reprinted as Equation (5.6). 


$$
M_{i} \frac{d^{2} \delta_{i}}{d t^{2}}+D_{i} \frac{d \delta_{i}}{d t}=P_{i}-\sum_{j=1, j \neq i}^{m}\left(C_{i j} \sin \delta_{i j}+D_{i j} \cos \delta_{i j}\right)
$$

The TEF (Equation (3.27)) explained in Section 3.2.1 has been reprinted as Equation (5.7) for clarity. The energy function shown in Equation (5.7) has been proposed for the simplified power system model represented in Equation (3.24) (when $\left.D_{i} \equiv 0\right)[48,51]$. when the the $m^{t h}$ synchronous generator is considered as the infinite bus, $\theta_{i}=\delta_{i}-\delta_{m}$ and $\tilde{\omega}=\omega_{i}-\omega_{m} . \theta_{i}^{s}$ is the respective rotor angle at the post fault operating point.

$$
\begin{aligned}
V(\theta, \tilde{\omega})= & \underbrace{\frac{1}{2} \sum_{i=1}^{m} M_{i} \tilde{\omega}_{i}^{2}}_{K E}-\underbrace{\sum_{i=1}^{m} P_{i}\left(\theta_{i}-\theta_{i}^{s}\right)}_{P E 1} \\
& -\underbrace{\sum_{i=1}^{m-1} \sum_{j=i+1}^{m}\left[C_{i j}\left(\cos \theta_{i j}-\cos \theta_{i j}^{s}\right)\right]}_{P E 2} \\
& +\underbrace{\sum_{i=1}^{m-1} \sum_{j=i+1}^{m}\left[\int_{\theta_{i}^{s}+\theta_{j}^{s}}^{\theta_{i}+\theta_{j}} D_{i j} \cos \theta_{i j} d\left(\theta_{i}+\theta_{j}\right)\right]}_{P E 3}
\end{aligned}
$$

As shown in Equation (5.6), the four terms $K E, P E 1, P E 2$ and PE3 corresponding to each generator are the candidate input features for machine learning. The three terms, except $P E 3$, can be evaluated analytically. The path dependant term, PE3, can be evaluated using the trapezoidal approximation $[9,52]$.

\subsubsection{Application of SVMs for Classification}

The goal of security assessment is to determine a boundary that separates secure and insecure data points in the most accurate manner. This boundary exists in a very high dimensional state space, and the boundary is non-linear. The concept of SVM is to transform the original coordinates into a new coordinate set so that the 
boundary of separation is linear. SVMs have demonstrated better performance over multi-layer perceptron networks for TSA of power systems [24]. Recently, researchers have obtained successful results by applying the SVM as the learning technique and the Radial Basis Function (RBF) as the kernel function for the power system transient stability classification problem $[17,23,24]$. Although the SVM is used as the machine learning technique in this thesis, the proposed method can be implemented with other candidate machine learning techniques.

Two linearly separable sets of data can be separated by using many different lines (linear boundaries). In the said situation, many machine learning techniques are capable of identifying a solution (i.e. a line). However, with the noisy data, the chances of misclassification are higher when the margin between the separating line and the dataset is narrow. The SVM minimizes the misclassification by finding this linear boundary to separate with the maximum margin. The linear boundary with the largest margin maximizes the distance to the closest point from both classes and is called the optimal separating hyperplane [68]. The SVM problem formulation and the application is briefly discussed in Appendix D. The LIBSVM toolbox [69] which is available in public domain was used to implement the SVM for the simulations reported during this thesis.

\subsubsection{Two Simultaneous Processes: Training and Assessment}

The electrical power generation levels and unit dispatch changes continuously with varying loads to maintain the power system in the normal state of operation. According to [7], in the normal state "all system variables are within the normal range and no equipment is being overloaded. The system operates in a secured manner and is able to withstand a contingency without violating any of the constraints". The range of deviation of operating point makes it difficult to train a single learned network that is capable of assessing the dynamic security of a practical power system. Such an attempt will end up in generating a huge amount of training data which are mostly 
irrelevant to a given operating state of a power system. Therefore, it is more practical to train a network for a given time window (for example 15 minutes) into the future. For example, if the system state is captured at 6.00 a.m., then the goal is to train a network valid for the time period from 6.15 a.m. to 6.30 a.m.. With the help of load forecasting, it is possible to determine the range of load variations applicable to a relatively small window of time.

This process can be implemented as a continuous process as shown in figure 5-1. During any period of time (for example between $t_{2}$ to $t_{3}$ ) following two simultaneous processes are happening.

1. the data generation and training of a new network capable of security assessment for the next period of time $\left(t_{3}\right.$ to $\left.t_{4}\right)$; and

2. security assessment using a network trained during the previous period of time $\left(t_{1}\right.$ to $\left.t_{2}\right)$.

On-line DSA involves capturing a real-time snapshot of the power system and simulating the dynamic behaviour of the power system for a list of credible contingencies. The results of the recently computed computational cycle can be used to determine the system security until the next computational cycle is completed. Shorter computational cycles enable capturing the operating state more frequently. Therefore, the assessment results are more relevant to the system operational state. When the application of machine learning is considered, the frequent training of networks requires relatively less complicated non-linearities to be captured by the learned network. Therefore, the training process is simplified. Further, it will minimise the generation of unnecessary training data which are irrelevant to the present operating conditions of the power system. Accordingly, training a network capable of security assessment for a short time interval is more effective, useful and practical. The process of producing a learned network is comprised of two main steps

1. database generation for training data; and 
Process A: Data generation and network training for the next period $t_{3}$ to $t_{4}$ using a snapshot captured at $t_{2}$

Process B: Security assessment using the network trained during the period $t_{1}$ to $t_{2}$

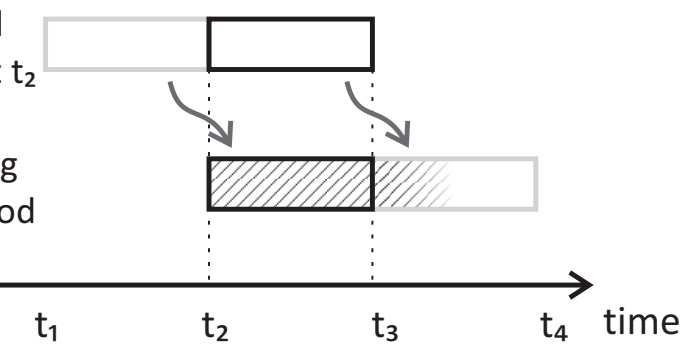

Figure 5-1: Two simultaneous processes 1) data generation and training a network for the next period of time 2) security assessment using the previously trained network

2. training of a network.

\section{Process A: Data Generation and Network Training}

This process, as shown in Figure 5-2, starts with creating the database of training data. Database generation requires the ranges of the system parameter deviation to be estimated. For a practical power system, the load changes during a computational cycle can be approximated using load forecasting. Usually the primary and secondary frequency controls respond to maintain the balance of load and generation by changing the power reference. This is facilitated by enabling a set of generators to change the power reference to cater to the mismatch between the loads and generation. Data generation starts with the generation of acceptable steady state operating points. The loading is changed randomly within the specified ranges of the deviation. Then, the power flow is solved and the bus voltages and controller parameters are checked. If all quantities are found to be within proper pre-determined ranges, that operating point becomes an acceptable operating point. If not, corrective actions are executed to bring the system parameters to acceptable values. If the corrective actions are capable of bringing the system parameters to normal values, the particular operating point becomes an acceptable operation point. Otherwise, that operating point is discarded. The steady state operating point generation procedure continues until the required number of operating points are generated. 


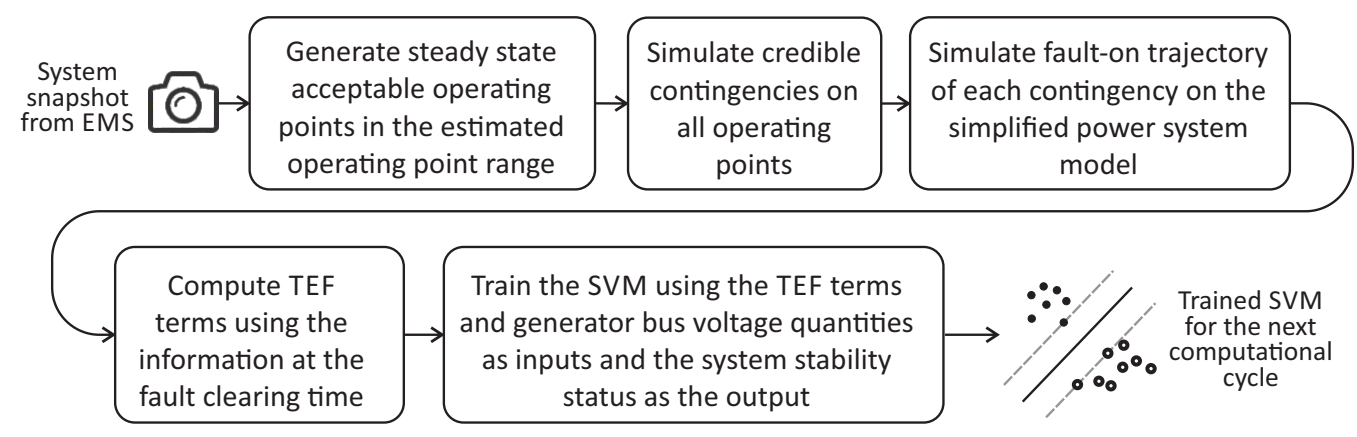

Figure 5-2: Process A: Flow chart for data generation and network training for the next computational cycle.

The next step is to perform the time-domain simulations and to determine whether the system is transiently stable or not for each credible contingency. The system stability state is the target value for training the network. The inputs are the energy terms computed at the fault clearing time. For the purpose of generating these inputs, a simplified power system model (classical generator model and the constant admittance load model) is simulated up to the fault clearing time. Four energy terms as indicated in Equation (5.7) are computed for each synchronous generator. The determination of the stability status requires the complete power system model with detailed generator models to be simulated for each and every contingency for the set of selected steady state operating points. Therefore, the database generation takes a significantly longer duration and more computing resources when compared to the training of a network.

\section{Process B: Security Assessment Using a Trained Network}

Algorithm (5.1) indicates the steps for security assessment using a trained network. The system snapshot is assumed to be received from the EMS. To prepare the inputs to the learned network, the simplified power system model is simulated up to the fault clearing time and the energy terms are computed based on the faulted trajectory information and the network structure. Then, the stability of the contingencies is 
estimated using the set of input features including the energy terms. This process is repeated for all contingencies. Moreover, the simulation of contingencies and the computation of the energy terms can be performed in parallel. That enables the implementation of the entire process to complete even faster. The system security state is determined after the stability of the system under all contingencies is assessed.

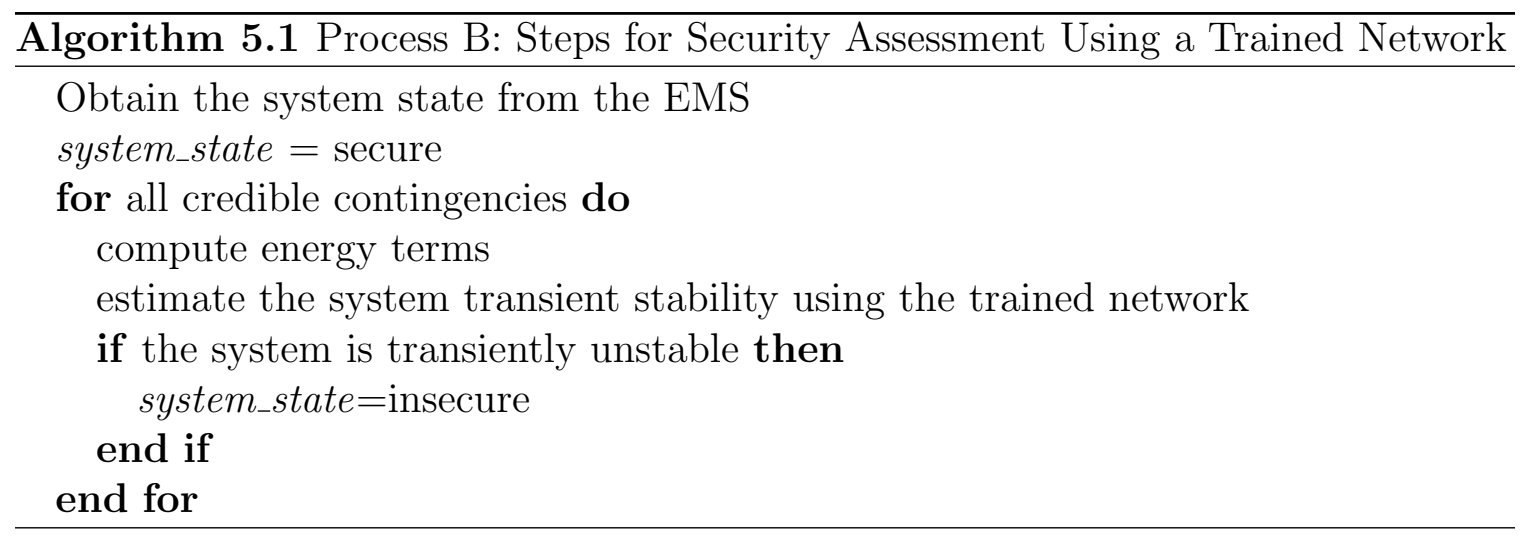

\subsection{Simulation and Results}

\subsubsection{Test System Model}

The performance of the proposed technique is evaluated using the New England 39bus test power system [70]. This test power system model has been used in studies and documented in the literature for testing TSA algorithms. The single line diagram of the test system is shown in Figure 5-3. This test system is comprised of 39 buses, 10 generating units, 19 loads, and 46 transmissions lines (Appendix C).

The system dynamics for TSA and for the energy term calculation are modelled as follows:

- For TSA: a) synchronous generator (fifth-order model); b) excitation system: exciter, stabilizing feedback, and terminal voltage transducer $c$ ) power system stabilizer consisting of washout filter and lag/lead phase compensation; and d) load model: constant admittances. 


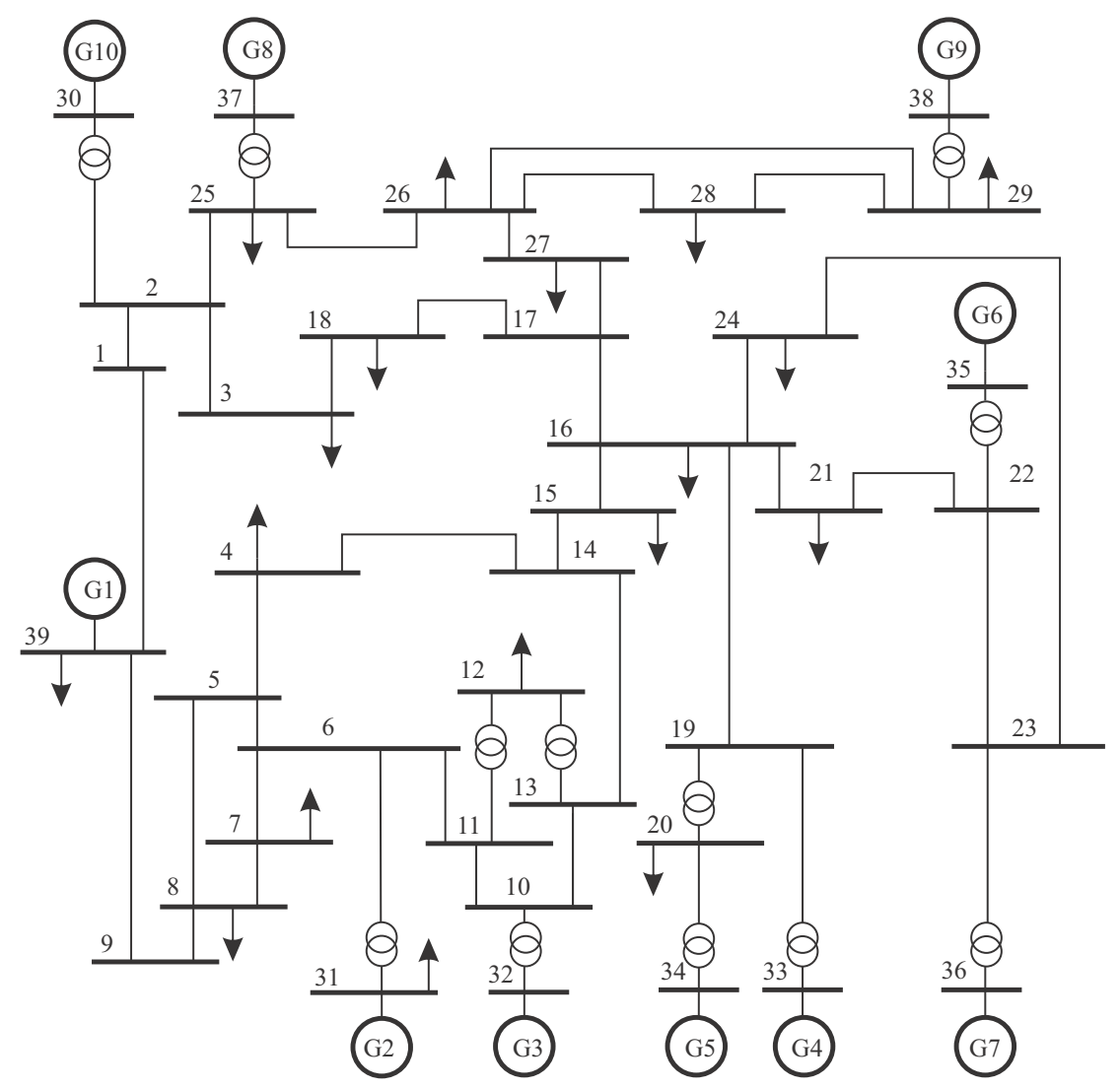

Figure 5-3: Single line diagram of the New England 39 bus 10 generator test power system

- For energy terms calculation: the generating units were modelled using the classical generator model and the loads were modelled as constant admittances. Further, the swing generator was modelled as an infinite bus.

\subsubsection{Data Generation}

In order to generate a representative and statistically sound dataset, the loads and the generation of the current operating point were changed. Active and reactive power of the loads were varied by $\pm 20 \%$ (i.e. $\Delta P^{(i)}=\Delta Q^{(i)}=0.2$ ) respectively using Equation (4.1) and Equation (4.2) [65]. $\varepsilon$ is a uniform independent random variable between 0 and 1 .

Active power generation and the terminal voltage of a generator bus were varied respectively using Equation (4.3) and Equation (4.4). Accordingly, the generators on 
bus 34 and 38 were allowed to change the power reference by $\pm 20 \%$ (i.e. $\Delta P^{(i)}=0.2$ ) and the terminal voltage by $\pm 2 \%$ (i.e. $\Delta V^{(i)}=0.02$ ) of the base case conditions.

Then, the power flow is solved with the changed loading and generation. The power mismatch is assumed to be supplied by the swing generator on bus 39 . If the power flow is converged, the general limit checks are performed on the new operating point to identify if that is an acceptable operating point. If the violations are identified, the particular operating point is discarded. If not, that particular operating point is considered as an acceptable operating point and is saved into the database. This process is repeated until 70 operating points are generated. The first 50 operating points were selected to generate the training dataset and the remaining 20 were used to validate the trained network.

After that, the stability state (i.e. 1 for stable and 0 for unstable) of each operating point for each credible contingency is assessed by performing a non-linear time domain simulation with the full detailed models of generators. This stability state is the target value in the dataset for training and testing of the SVM. The most severe type of fault, three phase to ground, is considered as the type of fault. Following contingencies were selected for simulations: 1) fault on the line 26-29 closer to the bus 26 cleared by isolating the line; 2) fault on the line 2-3 closer to the bus 2 cleared by isolating the line; 3) fault on the line 15-16 closer to the bus 16 cleared by isolating the line; 4) fault on the line 11-12 closer to the bus 12 cleared by isolating the line; and 5) fault on the line 16-21 closer to the bus 21 cleared by isolating the line. The faults were expected to be cleared by the primary protection, thus, a $100 \mathrm{~ms}$ fault clearing time was assumed. The other 5 contingencies are the same as above except the fault is assumed to be cleared by the back-up protection, thus, a $300 \mathrm{~ms}$ fault clearing time is assumed. Accordingly, the size of the training dataset is 500 (50 operating points $\times 10$ contingencies) and the size of the validation dataset is 200 (20 operating points $\times 10$ contingencies). The dynamic simulations are performed using the complete dynamic models (not the classical model) and the stability status is 
recorded in the built in database of the data generation software ML4DSA. At the end of the time domain simulation of the postfault system for a duration of $3 \mathrm{~s}$, the transient stability of the system under a particular contingency is concluded based on the power angle based stability index shown in Equation (5.8) as proposed in [40]. $\Delta \delta_{\max }$ is the absolute value of the maximum angle separation of any two generators at the end of the postfault system simulation. If $\eta>0$ the system is considered as transiently stable, otherwise, the system is transiently unstable. The system was classified as transiently stable for 249 contingencies and unstable for 251 contingencies in the training set. In the validation set, the system was transiently stable for 101 contingencies and unstable for 99 contingencies. The same validation set is used for the reported results in Section 5.4.4 and 5.4.5, therefore, the size of the validation set is not included in the tables.

$$
\eta=\frac{360^{\circ}-\Delta \delta_{\max }}{360^{\circ}+\Delta \delta_{\max }}
$$

The swing generator was modelled as an infinite bus during the fault-on trajectory simulation using the simplified power system model.

\subsubsection{Accuracy Indexes}

The classification accuracy is evaluated by the following indexes.

1. $\% F A=\left(\sum(\right.$ False Alarms $\left.) / n\right) \times 100$

2. $\% F D=\left(\sum(\right.$ False Dismissals $\left.) / n\right) \times 100$

3. $\% F C=\left(\sum(\right.$ False Alarms + False Dismissals $\left.) / n\right) \times 100$

where $n$ is the number of samples in the validation dataset. A FA occurs when a stable operating point for a particular contingency is classified as unstable, and a FD occurs when an unstable operating point for a particular contingency is classified as 
stable. A False Classification (FC) is a misclassification occurred due to a FA or a FC.

\subsubsection{Feature Selection for Machine Learning}

The cross validation accuracies obtained on two input feature sets based on the candidate feature sets mentioned in Section 5.3.2 are shown in Table (5.2). In the first set, the input features are the energy terms, thus, the number of terms is 36 (i.e. 4 terms per generator $\times 9$ generators). The other dataset has two inputs per generator (kinetic energy and sum of potential energy terms). In this dataset the total number of input features is 18 (i.e. 2 terms per generator $\times 9$ generators).

Table 5.2: 5 -fold cross validation accuracy of two feature sets based on the four TEF terms

\begin{tabular}{|l|c|l|}
\hline Input features & Dataset size & \% FC (5-fold CV) \\
\hline$K E, P E 1, P E 2, P E 3$ & \multirow{2}{*}{500} & 1.2 \\
\cline { 1 - 1 }$K E, P E 1+P E 2+P E 3$ & & 1.2 \\
\hline
\end{tabular}

The computation burden of computing the PE3 term is significantly higher than the other terms because it involves numerical integration. Therefore, the computational complexity could be further decreased if the network can be trained without using the PE3 term. The accuracy of the classification excluding the term PE3 presented in Table 5.3 indicates that the said term can be excluded from the input features without losing the accuracy. In addition to the results presented in the Table 5.3, numerous sensitivity studies were performed to determine the best set of input features. It was found that the input sets shown in Table 5.3 gave the best performance when only the energy terms are present in the input feature set.

Table 5.3: Training and validation accuracy of two networks trained without the numerically intensive $P E 3$ term

\begin{tabular}{|c|c|c|c|c|c|}
\hline \multicolumn{3}{|c|}{ Training } & \multicolumn{3}{|c|}{ Validation } \\
\hline Input features & Dataset size & $\% \mathrm{FC}(5$-fold CV) & $\% \mathrm{FA}$ & $\% \mathrm{FD}$ & $\% \mathrm{FC}$ \\
\hline$K E, P E 1, P E 2$ & 500 & 1.2 & 1 & 0.5 & 1.5 \\
\hline$K E, P E 1+P E 2$ & 500 & 1.2 & 1.5 & 0.5 & 2 \\
\hline
\end{tabular}


Generator bus voltage magnitudes and phase angles are identified as strong features for application of machine learning for TSA $[15,17]$. Table 5.4 shows the classification accuracy obtained using the two networks trained by incorporating the per unit generator bus voltage magnitudes and phase angles (in radians), obtained at fault clearing time, in the input features. Therefore, the feature sets in Table 5.4 are identified as a better combination of input features.

Table 5.4: Training and validation accuracy once the generator bus voltage related input features are incorporated

\begin{tabular}{|l|l|l|l|l|l|}
\hline \multicolumn{3}{|c|}{ Training } & \multicolumn{3}{c|}{ Validation } \\
\hline Input features & Dataset size & \% FC (5-fold CV) & $\% \mathrm{FA}$ & $\% \mathrm{FD}$ & $\% \mathrm{FC}$ \\
\hline $\begin{array}{l}K E, P E 1, P E 2, \quad V_{\text {mag }}, \\
V_{\text {ang }}\end{array}$ & \multirow{2}{K}{$\begin{array}{l}K E, P E 1+P E 2, \quad V_{\text {mag }}, \\
V_{\text {ang }}\end{array}$} & 1 & 1 & 0.5 & 1.5 \\
\cline { 3 - 7 } & & 1.5 & 1 & 0.5 & 1.5 \\
\hline
\end{tabular}

In order to demonstrate the effectiveness of using the energy terms, we trained a network with only the voltage magnitude terms and phase angles as input features. The best performance was $7.6 \%$ of FCs using the 5 -fold cross validation. This clearly indicates the improvement of assessment accuracy achieved by using energy terms along with generator bus voltage magnitudes and phase angles.

\subsubsection{Accuracy of Classification for Smaller Training Datasets}

This section determines whether the size of the training dataset can be reduced without sacrificing the accuracy of classification. The size of the dataset used for the results presented in Table 5.4 is 500 . The training process was repeated with smaller datasets of 250 and 125. The results are shown in Table 5.5. The number of contingencies in the training dataset for which the system was classified as stable $(\mathrm{S})$ and unstable $(\mathrm{U})$ are also given in the Table 5.5.

The results shown in Table 5.5 confirm the half-sized training dataset is capable of producing well trained networks. Although, there is an increase of classification error with respect to the original (500) dataset, the classification accuracy is acceptable. 
Table 5.5: Training and validation accuracy of networks trained using a half and a quarter sized training dataset

\begin{tabular}{|c|c|c|c|c|c|}
\hline \multicolumn{3}{|c|}{ Training } & \multicolumn{3}{|c|}{ Validation } \\
\hline Input features & Dataset size & \%FC (5-fold CV) & $\% \mathrm{FA}$ & $\% \mathrm{FI}$ & $\% \mathrm{FC}$ \\
\hline $\begin{array}{l}K E, P E 1, P E 2, V_{\text {mag }}, \\
V_{\text {ang }}\end{array}$ & 250 & 2.4 & 1 & 1.5 & 2.5 \\
\hline $\begin{array}{l}K E, \quad P E 1+P E 2, \quad V_{\text {mag }}, \\
V_{\text {ang }}\end{array}$ & (S: $125 \mathrm{U:}: 125)$ & 2.4 & 1 & 1.5 & 2.5 \\
\hline $\begin{array}{l}K E, P E 1, \quad P E 2, \quad V_{\text {mag }}, \\
V_{\text {ang }}\end{array}$ & & 4.8 & 2.5 & 1.5 & 4 \\
\hline $\begin{array}{l}K E, \quad P E 1+P E 2, \quad V_{\text {mag }}, \\
V_{\text {ang }}\end{array}$ & (S: 60 U: 65) & 3.2 & 2.5 & 1.5 & 4 \\
\hline
\end{tabular}

The quarter-sized training dataset (125) is still capable of producing networks with over $95 \%$ classification accuracy. The performance on the validation dataset indicates a proper generalisation of the trained networks. Further, the percentage of FDs shows no increase for a network trained using a half-sized or a quarter-sized dataset.

\subsubsection{Classification Accuracy for Unbalanced Faults with the Presence of Voltage Sensitive and Dynamic Loads}

The study was extended by introducing unbalanced faults in the presence of voltage sensitive and dynamic loads. The load characteristics affect the transient stability of a power system [47]. In order to assess the performance of the proposed algorithm in the presence of the voltage dependant and dynamic loads, the loads connected to bus $4,12,16$ and 18 were replaced by composite loads. The composite loads are comprised of $40 \%$ large induction generators, $20 \%$ small induction generators, $20 \%$ discharge lighting and $20 \%$ constant power load. This composition was selected based on the typical range of values mentioned in [47]. During this study, dynamic loads were included in the system model for TSA. The same model as in Section 5.4.1 was used for TEF term calculation.

Contingencies of single line to ground faults at the middle of following lines were included. 1) 26-29; 2) 2-3; 3) 15-16; 4) 11-12; and 5) 16-21. The faults were cleared by 
isolating the faulted line. Similar to the contingency specifications in Section 5.4.2, a faulted time of $100 \mathrm{~ms}$ was assumed for the faults cleared by the primary protection and $300 \mathrm{~ms}$ was assumed for the faults cleared by the backup protection. The sum of negative sequence fault impedance, $Z_{2}$, and the zero sequence fault impedance, $Z_{0}$, was used as the effective fault impedance, $Z_{e f}$, in the positive sequence model for TSA, as shown in Equation (5.9), in order to represent the effect of the single line to ground fault [7] .

$$
Z_{e f}=Z_{2}+Z_{0}
$$

Table 5.6 shows the classification accuracy of the networks trained using a dataset of size 250. The simulation was performed for two operating points. The first operating point was the same as previous. The second operating point was obtained by scaling-up system wide loads and generation by $10 \%$ to obtain a more stressed operating point. Table 5.7 shows the classification accuracy of the networks trained using a dataset of size 125 . The results confirm that the proposed method is valid in unbalanced fault situations and with the presence of the voltage sensitive and dynamic loads.

Table 5.6: Training and validation accuracy of networks trained using a dataset of 250 items for line to ground faults with the presence of voltage dependant and dynamic loads

\begin{tabular}{|l|l|l|l|l|l|}
\hline $\begin{array}{l}\text { Operating } \\
\text { point }\end{array}$ & Input features & $\begin{array}{l}\% \mathrm{FC}(5- \\
\text { fold CV })\end{array}$ & $\% \mathrm{FA}$ & $\% \mathrm{FD}$ & $\% \mathrm{FC}$ \\
\hline \multirow{2}{*}{1} & $K E, P E 1, P E 2, V_{\text {mag }}, V_{\text {ang }}$ & 0 & 0 & 1 & 1 \\
\cline { 2 - 6 } & $K E, P E 1+P E 2, V_{\text {mag }}, V_{\text {ang }}$ & 0 & 0 & 1 & 1 \\
\hline \hline \multirow{2}{*}{2} & $K E, P E 1, P E 2, V_{\text {mag }}, V_{\text {ang }}$ & 0.8 & 1 & 0 & 1 \\
\cline { 2 - 6 } & $K E, P E 1+P E 2, V_{\text {mag }}, V_{\text {ang }}$ & 0.8 & 0 & 2.5 & 2.5 \\
\hline
\end{tabular}

\subsubsection{Computational Performance and Resource Requirement}

Training data generation for the SVM mentioned in Table 5.5 (dataset size 125) required 2 minutes. The parameter search process was completed in $0.6 \mathrm{~s}$. The training 
Table 5.7: Training and validation accuracy of networks trained using a dataset of 125 items for line to ground faults with the presence of voltage dependant and dynamic loads

\begin{tabular}{|l|l|l|l|l|l|}
\hline $\begin{array}{l}\text { Operating } \\
\text { point }\end{array}$ & Input features & $\begin{array}{l}\% \mathrm{FC}(5- \\
\text { fold CV })\end{array}$ & $\% \mathrm{FA}$ & $\% \mathrm{FD}$ & $\% \mathrm{FC}$ \\
\hline \multirow{2}{*}{1} & $K E, P E 1, P E 2, V_{\text {mag }}, V_{\text {ang }}$ & 0 & 1 & 0 & 1 \\
\cline { 2 - 6 } & $K E, P E 1+P E 2, V_{\text {mag }}, V_{\text {ang }}$ & 0 & 0 & 1 & 1 \\
\hline \hline \multirow{2}{*}{2} & $K E, P E 1, P E 2, V_{\text {mag }}, V_{\text {ang }}$ & 1.6 & 1.5 & 0 & 1.5 \\
\cline { 2 - 7 } & $K E, P E 1+P E 2, V_{\text {mag }}, V_{\text {ang }}$ & 1.6 & 0 & 2.5 & 2.5 \\
\hline
\end{tabular}

of the SVM required $0.03 \mathrm{~s}$. A prediction using the trained SVM was completed in negligible time (reported $0 \mathrm{~s}$ ). A fault-on trajectory simulation for a particular contingency took less than $0.01 \mathrm{~s}$. The computing times reported are based on a Windows 7 PC with Intel Core i7 $3.4 \mathrm{GHz}$ processor with 8 GB RAM. Accordingly, with the reported hardware and for test power system, it is possible to implement a DSA system with a 3 minute computational cycle. The data generation process is comprised of highly parallelisable tasks (e.g.: stability status of a single steady state acceptable operating point under each contingency can be assessed as a stand-alone task). For larger power systems, the speed-up of the entire process can be achieved by introducing more computing resources.

\subsubsection{Inclusion of FACTS Devices Including HVDC Lines}

The proposed approach facilitates the stability assessment of power systems integrated with FACTS devices by introducing the device specific terms of the TEF to the input features of the SVM. For example, [71] presents the proposed energy function with additional terms to Equation (5.7) in order to represent the dynamics of a unified power flow controller (UPFC). An energy function for a static series synchronous compensator (SSSC) in the similar structure is reported in [72]. The method proposed in [73] can be used to incorporate the HVDC dynamics into the TEF by excluding the integral terms in the proposed TEF. 


\subsubsection{Significance of the Proposed Method}

This section discusses the comparative advantages of different machine learning based methods proposed in literature on the accuracy of the assessment, specifications of the dataset, speed of assessment and the specifications of the machine learning application. The classification accuracy reported in this thesis obtained for the IEEE 39 bus 10 generator test power system which is about $96 \%$ is comparable to the similar research carried out in recent past $[15,17,20]$. Moreover, the percentage of FDs, which is about $1.5 \%$, occurred in the proposed method is lower than the reported FDs in [15] and [17]. Research work proposed in [20] and [15] requires the contingency specific networks to be trained, whereas, the proposed technique requires only a single network to be trained for the set of credible contingencies. This is expected because the contingency specific information is already embedded in the energy terms used in the proposed method. In addition, the TEF features are capable of training networks with significantly smaller training datasets as shown in Table 5.5. For example, the technique proposed in [20] and [15] requires a dataset of 100 elements to train a network for each and every contingency. The technique proposed in [17] for protection applications, requires 492 elements in the dataset to train a network to detect the transient stability of the system. This improved performance was also expected because pre-processed input features were used in the proposed method (this was illustrated in the simple example in 5.3.1).

The duration and the model complexity of the time domain simulation required to compute the input features for the learned networks is significant in determining how fast the assessment can be made by a particular technique. The proposed technique requires only the fault-on trajectory (up to the fault clearing time) to be simulated for computing the input features. The range of the operating point deviation that can be accommodated for accurate stability assessments is also a strength in the proposed method. The proposed technique is capable of accommodating load and generation (real power) deviations in the range of $-20 \%$ to $+20 \%$. This is a wider 
range compared to the $-10 \%$ to $+10 \%$ captured in [15] and upto $+10 \%$ in $[17,23]$.

The duration of the post fault trajectory simulation which is performed in Process A, training data generation for machine learning, can be experimentally adjusted based on the system insights. In a context where multi-swing instabilities may occur, the initial step is to extend the duration, for example up to $10-20 \mathrm{~s}$, of the post fault trajectory simulation. That results in capturing the multi-swing unstable cases into the training dataset.

\subsection{Concluding Remarks}

The novel algorithm to enable more frequent assessment cycles in on-line DSA, based on energy based power system features and machine learning, proposed in this thesis was presented in this chapter. It was shown that the proposed method is capable of producing learned networks with significantly smaller sized datasets. The classification accuracy of the proposed algorithm was examined for different datasets and for different operating conditions. Further, the proposed algorithm was tested for unbalanced faults with the presence of voltage sensitive and dynamic load models. The results indicated that estimation accuracy is applicable for TSA.

The implementation details of the proposed technique which involves two simultaneously running processes was explained in detail. Integration of the proposed method for practical power systems was discussed in brief. The proposed method enables the application of machine learning based on a most recent operating point and for the operating point deviations estimated based on load forecasting that minimise the data generation on practically irrelevant operating conditions. Further, as a single trained network is in use only for a single computational cycle, the non-linearities to be captured by the machine learning is limited. This makes the learning process simple and fast. 


\section{Chapter 6}

\section{Implementation of on-line DSA}

\subsection{Introduction}

A DSA scheme comprises of many components dedicated to perform different functionalities. Some of these components already exist in the power system, whereas, the other components may be newly introduced. A practical implementation requires the integration of these components to work in a seamless manner.

By considering the computational demand of the automation and amount of data to be generated, a reasonable question one might ask, having agreed to the method proposed in Chapter 5, is that "can this be practically implemented to be an online DSA system?". The main objective of this chapter is to present the framework developed for real-time and integrated testing of the DSA algorithm presented in this thesis. This chapter also highlights the importance of real-time testing of DSA algorithms. Finally, the current stage of development and the proposed improvements for applications to large power system models are discussed. 


\subsection{The Proposed Framework}

The main challenge of the on-line DSA is to conclude the system security within an acceptable computational cycle time. Long computation cycles taken by the DSA algorithms produce obsolete security assessment results as the power system operating state continues to evolve with the changes in loads, generation and the disturbances.

The proposed DSA scheme in Chapter 5 comprises of two continuous processes named Process $A$ and Process B. Each of these processes comprise of several seamlessly integrated components. For example, at a glance, the Process A, which produces a learned network, generates the acceptable steady state operating points upon receiving the most recent snapshot of the power system. Then, the system stability state under each credible contingency is assessed for the set of previously generated steady state operating points.

The future developments of machine learning based DSA will more likely be in the direction of adapting and frequent training of new networks as indicated in Figure 5-1. Although the real-time implementation is demonstrated for the algorithm proposed in Chapter 5, it is equally applicable for testing any other DSA algorithm.

\subsubsection{Requirement Specification}

A practically integrated implementation of on-line DSA scheme needs to address the following key aspects:

- critical data communications aspects.

- computational hardware requirements; and

- capabilities and limitations of the existing tools available at present.

The general list of specifications is listed as follows. The developed framework should be able to: 
(A) seamlessly integrate the different functional components of the entire security assessment process (i.e.: real-time simulators, data conversion and generation, machine learning, security assessment, operator information systems, etc.).

(B) configure distributed processing over many computational units to achieve the target computational cycle time.

(C) conveniently configurable to different power system models and for testing different DSA algorithms.

(D) utilize the industry proven tools for real-time simulations, power flow and dynamic simulations; and

(E) facilitate data archiving for on-demand applications.

The main functional requirement is to be able to complete the computations within the acceptable computational time-which is about $15 \mathrm{~min}$. Accordingly, within a single computational cycle, the scheme should be able to:

- generate the training data set upon receiving the most recent system snapshot (for training) and to train an accurately classifying network (once in a computational cycle); and

- continuously assess the system security using the system snapshots generated by the EMS (usually in every $2 \mathrm{~min}$ ).

These functional requirements leads to the following set of specifications.

(A) real-time simulation of the power system model: the proposed scheme should be able to $a$ ) change the loading and generator dispatch according to a prespecified load profile; and $b$ ) produce the system snapshots for machine learning and security assessment purposes. 
(B) should be able to convert the power system snapshot (in the form of meter captures) generated by the real-time simulator into a compatible form for the data generation algorithms including the load data, generation data and the breaker status.

(C) should be able to produce a learned network for the next computing cycle. Following steps of the process were explained in Section 5.4.2 to (5.4.4). a) Generate acceptable steady state operating points $b$ ) simulate the list of credible contingencies and conclude the stability based on a stability assessment algorithm (explained in Section 4.6) c) simulate the fault-on trajectory and compute the energy terms at the fault clearing time; and $d$ ) train the learned network.

(D) perform the security assessment using a learned network; and

(E) communicate the system security state to the operator.

\subsection{Overall Structure of the Proposed Framework}

The overall block diagram of the framework is illustrated in Figure 6-1.

The power system is simulated in a real-time digital simulator (RTDS). The load variations in the real-time simulated power system are implemented using a prespecified system load curve. Similarly, the pre-specified generation dispatch schedule is used to alter the generation. This algorithm is described in Section 6.4. Section 6.5 describes the system snapshot generation algorithm.

Process A and Process B are two independently running processes in two (or more) processing units over the Local Area Network (LAN). Both processes are triggered by the availability of the most recently captured power system snapshot. The snapshot

produced by the Real Time Digital Simulator ${ }^{\circledR}$ (RTDS) is made available to these two processes in a dedicated folder over the LAN. Process A uses the snapshot for producing a DSA learned network for the next computational cycle. Process B uses 


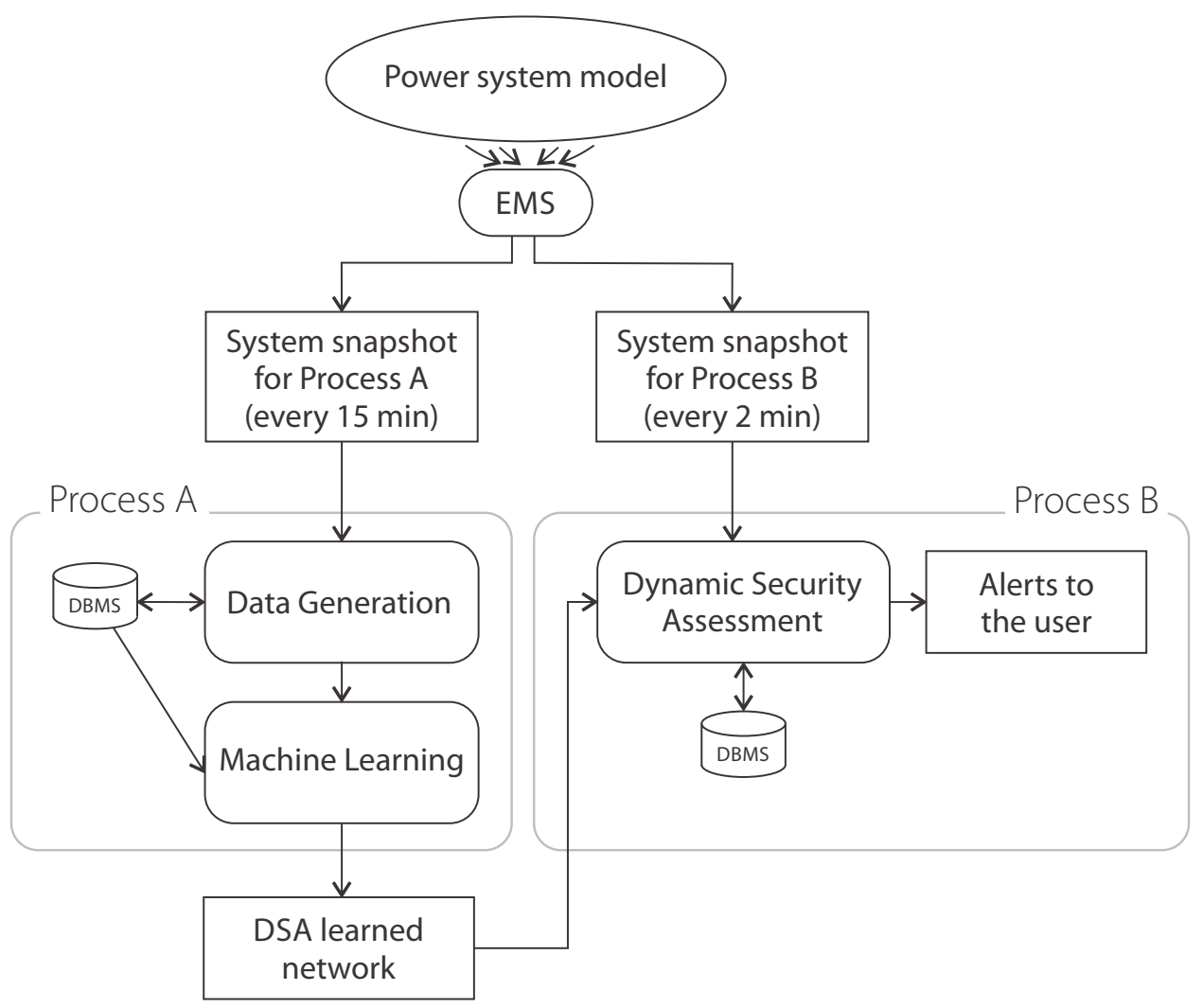

Figure 6-1: Overall block diagram of the real-time DSA framework

the snapshot for DSA by using the trained network produced by Process A.

The next stage of the process, which is common to both Process A and Process $\mathrm{B}$, is to convert the RTDS snapshot to a PSSE compatible power system snapshot. During this process, the base case power system model is altered to have the most recently captured values obtained from the RTDS snapshot. This process is designed in a way independent of the power system model. Then, the PSSE compatible power system snapshot is used for the production of a DSA learned network by Process A. The next two steps of the Process A are: 1) generate acceptable steady state operating points; and 2) system stability assessment of the set of operating points generated in step 1. The computation process of the energy features are common to Process A and Process B. Process A computes the energy features (of the system under each credible contingency and for all the operating points generated in step 1) for machine learning. Process B computes the energy features (for all credible 
contingencies using the most recently captured system snapshot) for DSA. The final step of Process A is to produce the DSA learned SVM using the generated data. The output of the Process A is made available to the Process B over a Network Attached Storage (NAS). Process B uses the output of the Process A, i.e. the DSA learned network, to estimate the system stability state under each credible contingency using the energy features as inputs.

The final stage of a computational cycle is to alert the system operator on the overall system security state under the current operating conditions. At present stage of development, if the system is insecure, the list of contingencies where the system is unstable is shown to the operator.

\subsection{Real-Time Simulation of Power System Oper- ation}

The practical power system operation is simulated in the RTDS. The key feature of the real-time simulators is to ensure the system simulation results are available in real-time. Further, the said feature demands for specific hardware requirements based on the power system model being simulated. The implementation of the complete IEEE 39-bus 10-generator system required 5-PCB processing cards. Two RTDS racks were connected using a point to point Global Bus Hub (GBH) connection [74]. Data

communication is facilitated by the Inter Rack Communication (IRC) interface which is built-in to GTWIF cards. The split of the power system model into two subsystems is as shown in Figure 6-2. 


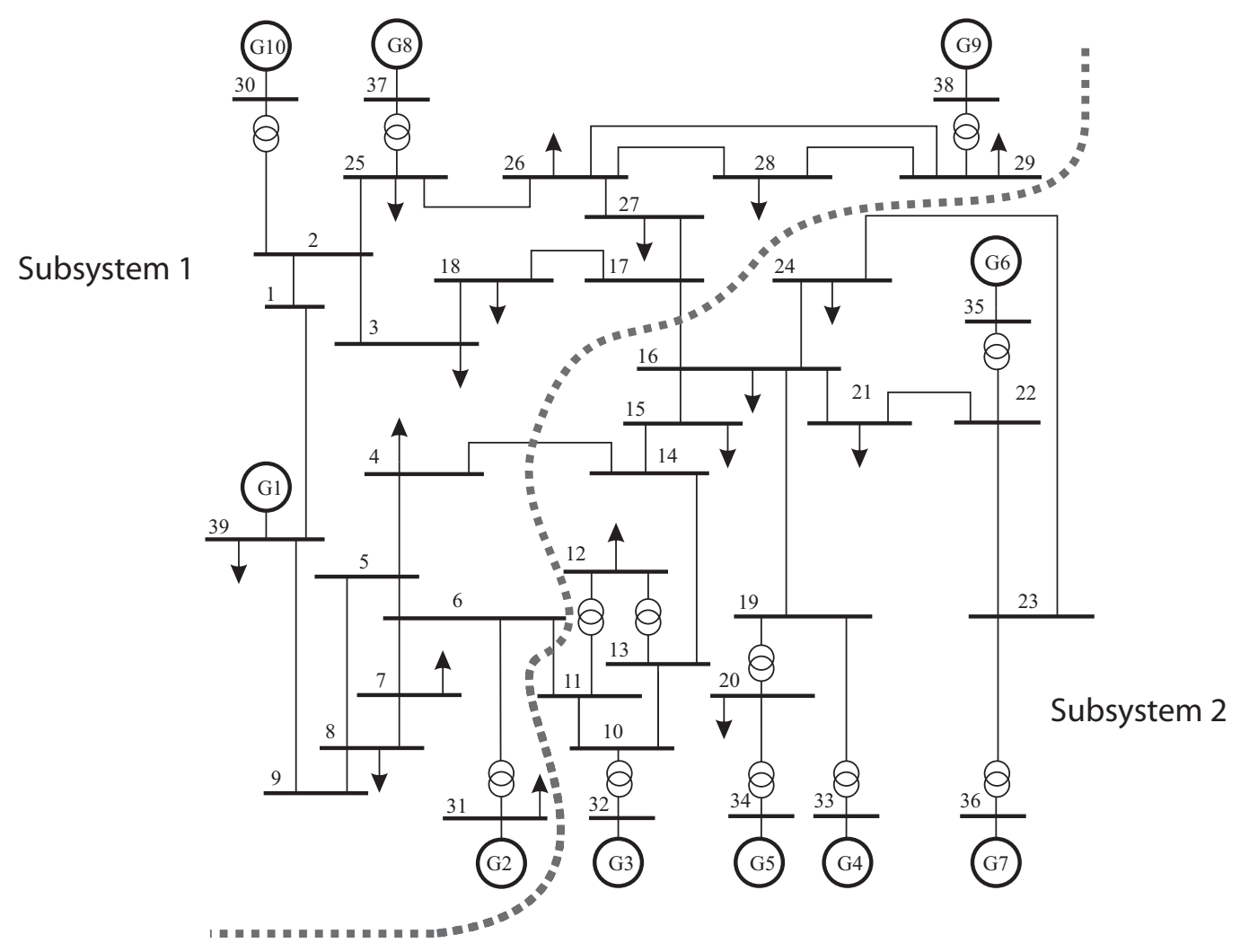

Figure 6-2: Boundaries of the two subsystems implemented on two RTDS racks (IEEE 39-bus 10 -generator test power system)

RTDS is an Electro Magnetic Transient (EMT) simulator. Transmission lines are modelled using the Bergeron model [75]. Further, the system is modelled as a three phase system.

The power system operating point continuously evolves due to the continuously occurring loading changes. It is important to test the DSA algorithms under changed operation conditions. Load forecasting provides accurate estimations of the load changes in a practical power system. Operating point deviation is facilitated by changing the power system loads according to a given load curve as shown in Figure 6-3. In Figure 6-3, the load change is represented by the fraction of the base case load.

Generation changes are implemented by changing the power reference of the generator governor. In practical power systems the generator/s (one or a small group 


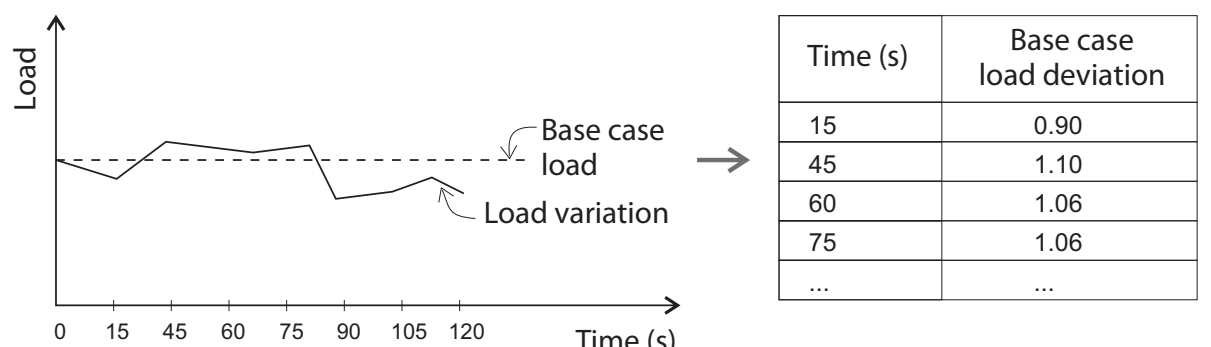

Figure 6-3: Representation of the load curve in $15 \mathrm{~s}$ intervals for a single load

of generators) on Automatic Generation Control (AGC) mode are responsible for changing the power reference to cater for the power mismatch. However, a generation re-dispatch can be ordered by the power system operators due to economic or maintenance purposes. These situations can be modelled in a similar manner.

\subsection{Construction of the System Snapshot}

Process A and Process B are triggered by the availability of the most recent power system snapshot in the dedicated folders in the LAN. The script running on RTDS exports the system snapshots in $15 \mathrm{~min}$ and $2 \mathrm{~min}$ time intervals respectively for Process A and Process B. Algorithm (6.1) is the pseudo code algorithm of the script running on RTDS. Component 1 and Component 2 are executed simultaneously using RTDS timer components by the time intervals defined for process $\mathrm{A}(15 \mathrm{~min})$ and process $\mathrm{B}(2 \mathrm{~min})$.

A power system snapshot is generated using the measurements of voltages, currents, breaker states, power flows, etc., collected from all over the power system components having the measurement units installed. A power system snapshot is typically prepared by the EMS by utilizing the measurements collected by the SCADA system. Integration of PMUs enable the production of more accurate snapshots by using time aligned data collected over the Wide Area Measurement System (WAMS). In practical systems, the EMS output is usually a solved power flow. 
Algorithm 6.1 Snapshot generation process

Input: meter_names.tex

Output: snapshot.csv

Component 1 Snapshot for process A

from meter_names.tex as meters

Create the empty snapshot.csv in the dedicated storage location on the LAN

foreach meter in meters do

Capture the reading from RTDS runtime as value

Insert a record to the snapshot file. record format: [meter, value]

end

\section{End Component}

Component 2 Snapshot for process B

Same as Component 1, except the change of the location of snapshot.csv corresponding to process B

\section{End Component}

In the developed framework, each entry of the generated snapshot (snapshot.csv) corresponds to the instantaneous value of a meter in the RTDS runtime environment. Each row of the snapshot.csv, as shown in Figure 6-4, comprise of three mandatory fields (Meter Name, Type and Bus) and an optional field (Id). A meter is assigned to measure 1) active power generation of a generator 2) reactive power generation of a generator 3) bus voltage magnitude 4) bus voltage angle 5) active power consumption of a load 6) reactive power consumption of a load ; or 7) plant voltage magnitude. The set of captured meters are selected based on the necessity of the measurements for the construction of the system snapshot. The captured readings of the meters are used to change the respective quantities of the base case power system model. This current development state is limited to facilitate only the load and generation changes of the base case, thus, allows no structure changes for a snapshot captured during on-line operation. This feature can be enabled by capturing the breaker status and applying those in the base case snapshot similar to changing the loads and generations. The names of the meters of which the values to be captured are given as an input to the RTDS script. This parametrization process makes the snapshot capturing process independent of the power system model. 


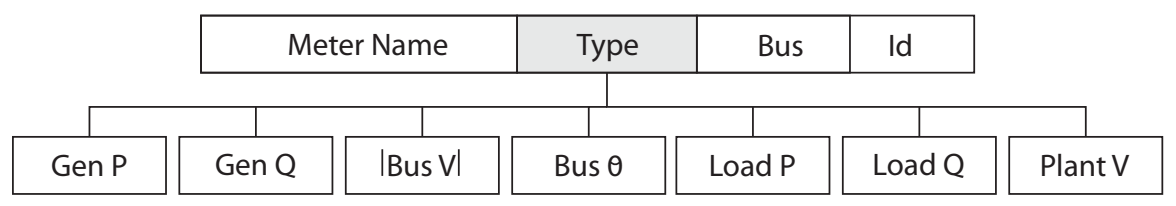

Figure 6-4: The format of a meter capture record in snapshot.csv and the 7 types of faciltated quantities

The snapshot created by the RTDS is to be converted into the PSSE compatible form to make it accessible by Process A and Process B (to automate the PSSE for training data generation and energy features computation). The inputs and output of the conversion process is shown in Figure 6-5. The algorithm should be capable of identifying the corresponding device type by the name of the meter where the value is captured in the snapshot.csv. This is facilitated by introducing an intermediate input meter_names_to_components which comprises of the information required to alter the respective value in the base case snapshot.

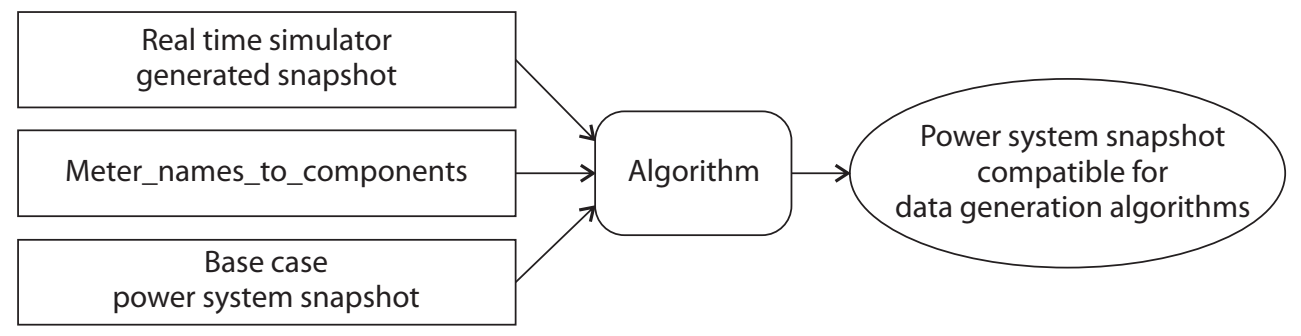

Figure 6-5: Representation of the load curve in $15 \mathrm{~s}$ intervals for a single load

Algorithm (6.2) shows the overall process flow of the snapshot conversion process. The Python based PSSpy Application Programming Interface (API) [64] is used to alter the base case parameters of respective components according to the RTDS snapshot. This process is independent of the power system model in use, therefore, only the input meter_names_to_components to be set appropriately for testing a different power system model. In addition, the snapshot generated by the RTDS and the PSSE compatible snapshots are stored in the built-in database for archiving purposes. 


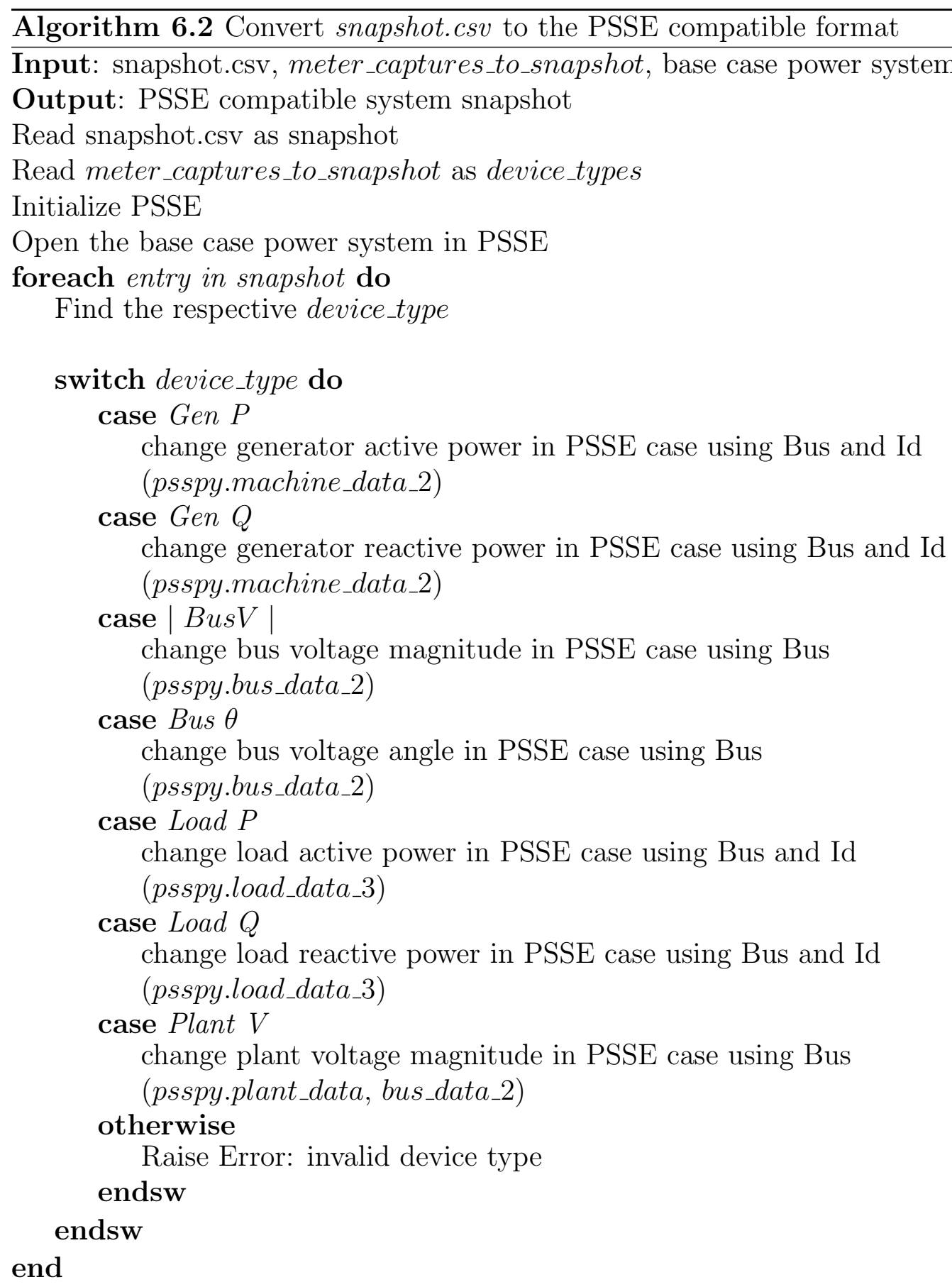




\subsection{Process A: Producing a Learned Network}

The developed framework seamlessly integrates the functionality of the following phases of process $A$ :

1. generation of steady state acceptable operating points.

2. simulation of credible contingencies and system stability state computation of each operating point.

3. fault-on trajectory simulation of simplified power system model.

4. computation of energy features using the fault-on trajectory; and

5. training the SVM.

\subsubsection{Scalability of Process A}

Process A comprises of highly computationally intensive tasks. For a practical power system, performing the time domain simulation of each credible contingency on each operating point is a highly time consuming process. For a power system suspicious of having multi-swing instabilities, the time domain numerical integration has to be performed for about $20 \mathrm{~s}$. With the computing power available in today's computers, it is challenging to ensure the computational cycle time while simulating all contingencies in a single computing unit. Simulating a single contingency on an operating point is a stand-alone task. Likewise, the step 2, in Section 6.6, comprises of a set of stand-alone processing tasks, thus, parallel processing can be conveniently introduced to achieve the speed-up (i.e.: by introducing more computing units). Therefore, for practical and large power system models the computational cycle time can be assured by introducing more computing units in step 2. This strategy is also applicable to step 1. 


\subsection{Process B: Security Assessment Using the Learned Network}

Process B computes the dynamic security status based on the most recent system snapshot and alerts the power system operator about the system security state. This entire process is explained in Section 5.3.4. The developed framework seamlessly integrates the functionality of following phases of Process B:

1. fault-on trajectory simulation of simplified power system model

2. computation of energy features using the fault-on trajectory

3. DSA estimation

4. alerting the operator

Once the system security state is computed, the next two steps are to

1. alert the power system operator

2. initiate the corrective actions

At present, the developed framework is capable of alerting the user on the system dynamic security state. If the system is insecure, the list of contingencies for which the system is unstable is shown to the user. The corrective actions may be initiated with or without the intervention of the system operator. Implementation of corrective actions is out of scope of this thesis.

\subsection{Concurrent Implementation of Process A and Process B}

At the present state of development, process $\mathrm{A}$ and process $\mathrm{B}$ are running in two computers. These two processes share the data over the LAN. The real-time simulator 
runs on two RTDS racks and the snapshots are exported to an NAS. The overall implementation is illustrated in Figure 6-6.

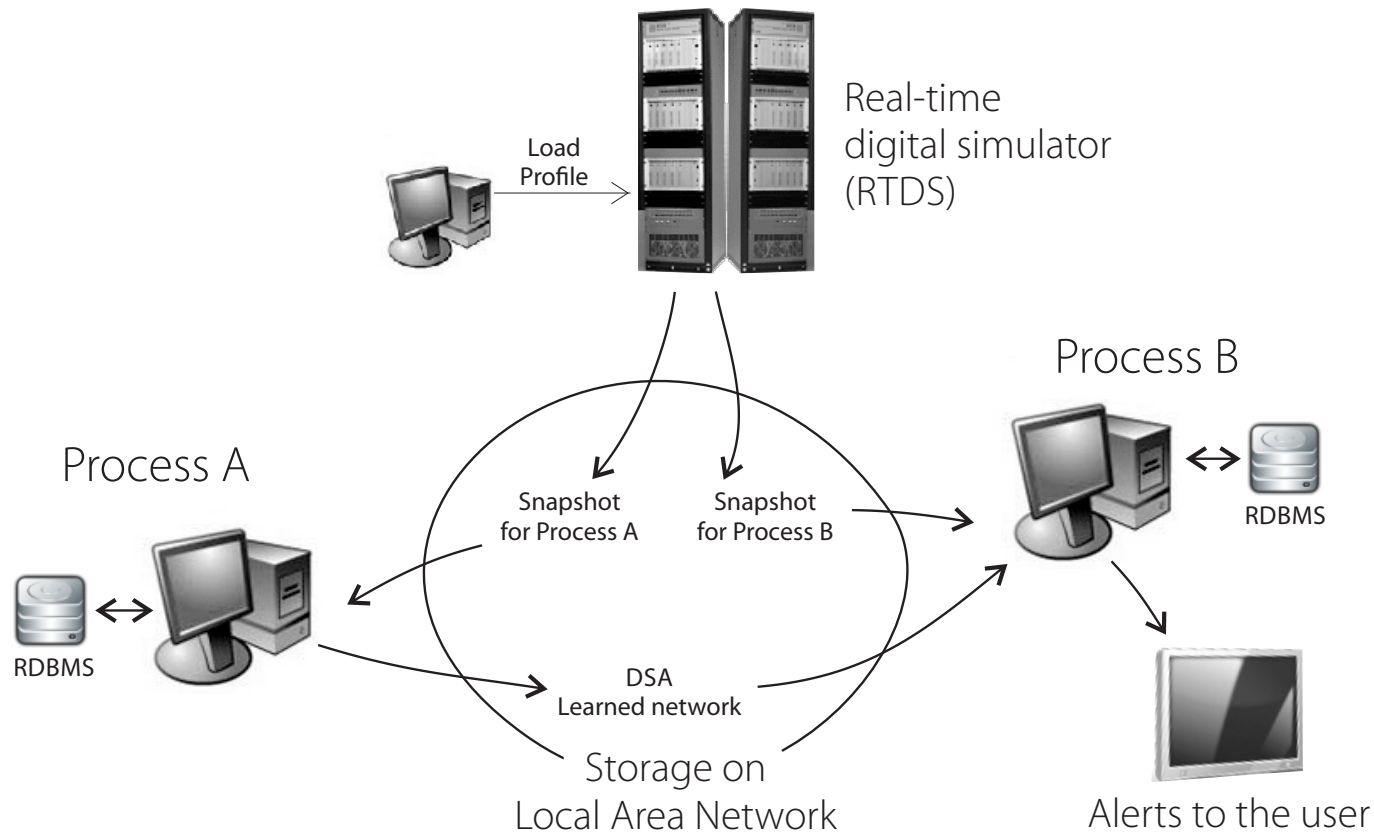

Figure 6-6: Illustration of the real-time implementation of DSA

Process A and B continuously checks for the availability of new snapshots. Once a snapshot is available, the respective process is triggered.

\subsection{Continuation at a Network Topology Change}

In on-line operation, the security assessment is performed using the most recently captured system snapshot. The assessment results will indicate if the system has moved to a new operating state such as alert or emergency. [6]. These information can be used to alert the operator or to trigger the controller actions. However, once the system is subjected to a contingency, the network structure is changed and data generated so far (to produce the learned network for the next period of time) becomes irrelevant for the new operating point. Therefore, the ongoing data generation should be paused and restated with respect to the new operating point. A similar situation 
arises at a generation re-dispatch or at a scheduled maintenance.

\subsection{Other features}

\subsubsection{DBMS}

Both Process A and Process B have access to built-in relational DBMSs. The framework is configured to archive information at different stages of the process. For example, the snapshots received from the RTDS and the PSSE compatible snapshot are archived in the database. This information is available to extended application development and for data mining purposes.

\subsubsection{User Authentication}

The proposed framework automates commercial software. Therefore, only the users who have been granted the privileges to access these software are eligible to operate the framework. The built-in user authentication module can be configured according to the software access policies of an organization.

\subsubsection{Web-based Interface}

The operator interface to the developed framework is a web interface and is based on web2py framework [63]. Accordingly, no software installations are required on the client computer.

\subsection{Testing}

During all stages of the development process, unit testing was performed on functional components to verify the functional requirements. Comprehensive complexity 
analysis has been performed during the development of each module to achieve the optimum performance.

Several tests were performed to ensure the fulfilment of the requirement specifications mentioned in Section 6.2.1 with a focus on the following aspects a) the capability of maintaining a consistent computation cycle time; b) to check if any incompatibilities exist among integrated functional components; and c) data communication issues. The test system, data generation, machine learning and the contingency specifications of the tests performed are based on the specifications provided in Section 5.4. An average of 2.5 min computation cycle time was observed during a seven hours of continuous operation of the framework. Maximum computation cycle time recorded is $2 \mathrm{~min}$ and $40 \mathrm{~s}$.

\subsubsection{Further Developments}

- Introduction of additional computing resources to speed-up the system stability assessment under the set of contingencies requires the development of process allocation algorithms. These algorithms are expected to divide the number of stand-alone simulations among the available computing resources.

- At present, the RTDS snapshot entries are used to alter the base case power system model which is available in the database. However, the application is to be developed to enable communication of the structural changes occurred in the base case in system model. For example, as a result of a scheduled maintenance event, one or more transmission line outages can be observed in the power system in operation. These scenarios can be facilitated by communicating the breaker status using the RTDS snapshot. The current state of the program requires the base case to be loaded with the particular line is disconnected.

- SVM training algorithms that are currently running in Matlab can be integrated to the ML4DSA which is based on Python. 
- It has been observed that the data file handling related to the Step 2 of Process A introduces significant delays to the entire process. The time series values of the recorded channels which are stored by the PSSE are read once again to assess the system stability status using the stability assessment algorithm. The same process for the fault-on trajectory happens in the steps 3 and 4 of Process

A and in steps 1 and 2 of Process B. A significant speed-up can be achieved if the data transfer between two processes is developed to be more efficient.

- The scope of this research is to monitor the dynamic security status of a power system in terms of the transient stability. The next step is to decide and apply the corrective actions to bring the system to the normal state, if the system state is insecure.

- Develop algorithms for on-line assessment of voltage stability and small signal stability.

\subsection{Concluding Remarks}

The implementation details of the algorithm proposed for on-line DSA in this thesis were presented in this chapter. The test power system model has been simulated using a RTDS. Implementation details of process $A$ and process $B$ were presented in detail. During a 7 hour long continuous operation, using the test power system model, a 2.5 min computational cycle time has been achieved. The techniques to achieve short computational cycles for large power system models were discussed. The overall organisation of the proposed framework over the LAN was discussed.

The real-time implementation framework was designed to enable convenient configuration for testing of different on-line DSA algorithms for different power system models. Implementation of a real-time security assessment scheme requires the integration of several functional components. This framework can be used to evaluate 
the performance and to assess the processing infrastructure requirements in a practical implementation in order to achieve a desired computational cycle time. The utilization of available computational tools and the networking infrastructure was also investigated. 


\section{Chapter 7}

\section{Conclusions, Contributions and Suggestions for Future Work}

\subsection{Conclusions}

The investigation of a TSA algorithm to enable frequent computational cycles in on-line DSA has resulted in following conclusions:

- For the application of TSA of power systems, the use of non-path dependant terms of the TEF along with the generator bus voltage magnitudes and the angles as input features to machine learning, enables the training of accurately classifying networks with significantly small sized datasets.

- A learned network produced as above is capable of predicting the power system transient stability under multiple contingencies. Also, the prediction accuracy remains acceptable for on-line DSA for unbalanced faults with the presence of voltage-sensitive and dynamic loads.

- The use of TEF terms as input features to machine learning enables the proposed technique to produce learned networks capable of making accurate predictions of system stability over a larger range of operating point deviation when 
compared to the methods that have already been proposed in literature.

- The computationally complicated path dependant terms of the TEF that demands for numerical integration can be excluded from the input feature set without losing the estimation accuracy.

- It has also been demonstrated that the proposed approach requires a significantly smaller set of training data due to: a) the network is trained for a recently captured snapshot of the power system in operation and b) the load forecasting information is used to specify the load and generation deviation during a given period of time to which a network has been trained for DSA.

- Power system devices such as HVDC and FACTS can be included in the proposed algorithm by incorporating the appropriate terms of the device specific TEF. The steps to follow have been highlighted in the thesis.

- An on-line DSA system can be implemented by using the method proposed in this thesis. The investigations confirmed that the following issues have been successfully addressed: a) critical data communications aspects; $b$ ) capabilities of computational hardware at present; and c) the capabilities and limitations of the existing tools to be used in automation.

The main contributions of this thesis are as follows:

- An algorithm based on energy based power system features to enable more frequent computational cycles in on-line DSA.

- Design and implementation of an on-line DSA system based on the algorithm proposed in this thesis (by integrating the following functional entities distributed over the LAN: real-time simulated power system model with changing load and generation based on load forecasting; simplified EMS; and data generation and security assessment algorithms running on distributed computers on the LAN). 
- Development of an integrated framework for testing the on-line DSA algorithms that can be configured for testing different DSA algorithms for different power system models.

- Design and development of a stand-alone software system for data generation for power system DSA studies.

These contributions have led to following publications:

J. Geeganage, U. Annakkage, T. Weekes, and B. Archer, "Application of energybased power system features for dynamic security assessment," Power Systems, IEEE Transactions on, vol. 30, no. 4, pp. 1957-1965, July 2015

J. Geeganage, U. Annakkage, M. Weekes, and B. Archer, "Application of energybased power system features for dynamic security assessment," in Power Energy Society General Meeting, 2015 IEEE, July 2015, pp. 1-1

J. Geeganage, U. Annakkage, B. Archer, T. Weekes, and H. Meiklejohn, "Realtime testing of dynamic security assessment algorithms," in CIGRE International Symposium, Lund, Sweden, May 2015

J. Geeganage and U. D. annakkage, "Estimation of transient energy terms using machine learning," in 2014 CIGRE Canada Conference, 2014

J. Geeganage, U. Annakkage, B. Archer, and T. Weekes, "A web based software system for database generation for online dynamic security assessment studies (ml4dsa)," in Electrical and Computer Engineering (CCECE), 2013 26th Annual IEEE Canadian Conference on, 2013, pp. 1-4

D. P. Wadduwage, J. Geeganage, U. D. Annakkage, and C. Q. Wu, "Investigation of the applicability of lyapunov exponents for transient stability assessment," in Electrical Power Energy Conference (EPEC), 2013 IEEE, Aug 2013, pp. $1-6$ 


\subsection{Suggestions for Future Work}

- This thesis identified the methodology of incorporating the FACTS and HVDC devices, however, further investigation is necessary on selecting the effective input features of the appropriate energy functions that are proposed in literature. Then, the DSA algorithm needs to be updated to accommodate these features to allow the DSA of power systems with these devices.

- Further study is to be carried out to identify the extent and the techniques to represent the external systems as dynamic equivalents. The classification accuracy of the proposed algorithm is recommended to be tested with the presence of dynamic equivalents.

- It is recommended to investigate effective techniques to enable the reuse of already generated training data to reduce the computational burden on Process A which will eventually result in further shortening the computational cycle time. Data mining is a potential technique to investigate in this context. At the beginning of the data generation cycle, if data mining can be used to discover the existing valid operating points in a database of archived training data, the dynamic simulation results corresponding to that particular operating points qualify to represent the current training dataset. The saving of computational time and the resources is expected to be significant.

- This thesis showed the possibility of introducing parallel processing into Process $A$, however, further developments are to be carried out to integrate this feature into the proposed scheme of on-line DSA. The algorithms to be developed to distribute the set of acceptable steady state operating points generated at the beginning of Process A, across a cluster of computational units over the LAN and to acquire the system stability status under each contingency once the dynamic simulations and the stability assessment is done. 
- It has been noted that a significant time during the data generation is spent on writing and reading the time-series data of dynamic simulations in the form of text files. The entire process can be performed faster if an efficient data transfer method is developed between the data generation software (ML4DSA) and the commercial software PSSE. 


\section{Appendix A}

\section{Derivation of the TEF for 3-Bus}

\section{Test Power System}

Using Equation (3.25)

$$
=\sum_{i=1}^{3}\left[M_{i} \dot{\tilde{\omega}}_{i}-P_{i}+P_{e i}+\frac{M_{i}}{M_{T}} P_{C O I}\right] \dot{\theta}_{i}, \quad i=1, \ldots, m
$$

The TEF is derived for the post fault power system. Assuming the generator rotor speed deviation is zero (i.e. $\tilde{\omega}=0$ ), generator rotor angle, $\theta^{s}$, at the post fault SEP is found by solving the non-linear algebraic equations (power flow solution). The rotor

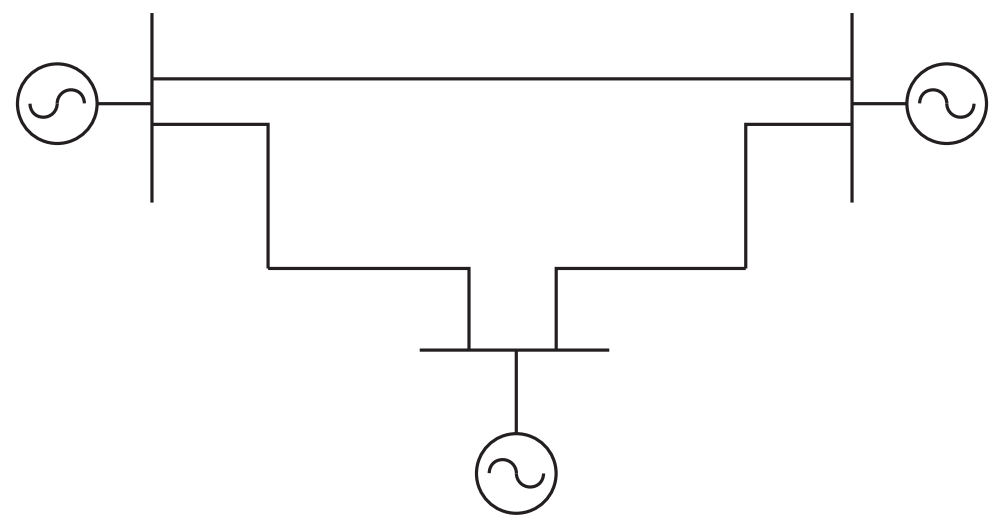

Figure A-1: A diagram of the reduced 3-machine 9-bus test power system model 
angles at the fault clearing time is assumed as $\theta^{t c l}$. Accordingly, the energy acquired at the fault clearing time is obtained by integrating Equation (A.1) between $\left(\theta^{s}, 0\right)$ and $\left(\theta^{t c l}, \tilde{\omega}^{t c l}\right)$.

$$
\begin{array}{r}
\underbrace{\sum_{i=1}^{3} M_{i} \dot{\tilde{\omega}}_{i} \dot{\theta}_{i}}_{\text {term } 1}-\underbrace{\sum_{i=1}^{3} P_{i} \dot{\theta}_{i}}_{\text {term } 2}+\underbrace{\sum_{i=1}^{3} \sum_{j=1, j \neq i}^{m} C_{i j} \sin \theta_{i j} \dot{\theta}_{i}}_{\text {term } 3} \\
+\underbrace{\sum_{i=1}^{3} \sum_{j=1, j \neq i}^{m} D_{i j} \cos \theta_{i j} \dot{\theta}_{i}}_{\text {term } 4}+\underbrace{\sum_{i=1}^{3} \frac{M_{i}}{M_{T}} P_{C O I} \dot{\theta}_{i}}_{\text {term } 5}
\end{array}
$$

Following section illustrates the simplification of each term of the Equation (A.1) for clarity.

Term 1:

$$
\begin{aligned}
& =\sum_{i=1}^{3} \int_{\theta^{s}}^{\theta^{t c l}} M_{i} \dot{\tilde{\omega}}_{i} \dot{\theta}_{i} \\
& =\sum_{i=1}^{3} M_{i} \int_{\tilde{\omega}^{s}}^{\tilde{\omega}^{t c l}} \omega_{i} \dot{\tilde{\omega}}_{i} \\
& =\sum_{i=1}^{3} M_{i}\left[\frac{1}{2} \tilde{\omega}_{i}^{2}\right]_{\tilde{\omega}^{s}}^{\tilde{\omega}^{t c l}}, \text { considering } \tilde{\omega}_{i}^{s}=0 \\
& =\sum_{i=1}^{3} \frac{1}{2} M_{i}\left(\tilde{\omega}^{t c l}\right)^{2}
\end{aligned}
$$

Term 2:

$$
\begin{aligned}
& =\sum_{i=1}^{3} \int_{\theta^{s}}^{\theta^{t c l}} P_{i} \dot{\theta}_{i} \\
& =\sum_{i=1}^{3} P_{i}\left(\theta^{t c l}-\theta^{s}\right)
\end{aligned}
$$




\section{Term 3:}

$=\sum_{i=1}^{3} \sum_{j=1, j \neq i}^{m} C_{i j} \sin \theta_{i j} \dot{\theta}_{i}$

$=C_{12} \sin \theta_{12} \dot{\theta_{1}}+C_{13} \sin \theta_{13} \dot{\theta_{1}}+C_{21} \sin \theta_{21} \dot{\theta_{2}}+C_{23} \sin \theta_{23} \dot{\theta_{2}}+C_{31} \sin \theta_{31} \dot{\theta_{3}}+C_{32} \sin \theta_{32} \dot{\theta_{3}}$ considering $\sin (-\theta)=-\sin \theta$, and $C_{i j}=C_{j i}$

$=C_{12} \sin \theta_{12}\left(\dot{\theta_{1}}-\dot{\theta_{2}}\right)+C_{13} \sin \theta_{13}\left(\dot{\theta_{1}}-\dot{\theta_{3}}\right)+C_{23} \sin \theta_{23}\left(\dot{\theta_{2}}-\dot{\theta_{3}}\right)$

$=C_{12} \sin \theta_{12} \dot{\theta_{12}}+C_{13} \sin \theta_{13} \dot{\theta_{13}}+C_{23} \sin \theta_{23} \dot{\theta_{23}}$

$=\int_{\theta_{i j}^{s}}^{\theta_{i j}^{t c l}} \sum_{i=1}^{2} \sum_{j=i+1}^{3} C_{i j} \sin \theta_{i j} \dot{\theta_{i j}}$

$=\sum_{i=1}^{2} \sum_{j=i+1}^{3}-\left[C_{i j} \cos \theta_{i j}\right]_{\theta_{i j}^{s}}^{\theta_{i j}^{t c l}}$

$=-\sum_{i=1}^{2} \sum_{j=i+1}^{3}-C_{i j}\left(\cos \theta_{i j}^{t c l}-\cos \theta_{i j}^{s}\right)$

Term 4: Similar to term 3, by considering $\cos (-\theta)=\cos \theta$

$$
\begin{aligned}
& =\sum_{i=1}^{2} \sum_{j=i+1}^{3} D_{i j} \cos \theta_{i j}\left(\dot{\theta}_{i}+\dot{\theta}_{j}\right) \\
& =\int_{\theta_{i}^{s}+\theta_{j}^{s}}^{\theta_{i}^{c l}+\theta_{j}^{c l}} \sum_{i=1}^{2} \sum_{j=i+1}^{3} D_{i j} \cos \theta_{i j} d\left(\dot{\theta}_{i}+\dot{\theta}_{j}\right)
\end{aligned}
$$

Term 5: According to the following simplification, the contribution of term 5 
becomes 0 .

$$
\begin{aligned}
& =\sum_{i=1}^{3} \frac{M_{i}}{M_{T}} P_{C O I} \dot{\theta}_{i} \\
& =\frac{P_{C O I}}{M_{T}}\left[M_{1} \dot{\theta_{1}}+M_{2} \dot{\theta_{2}}+M_{2} \dot{\theta_{2}}\right] \\
& =\frac{P_{C O I}}{M_{T}}\left[M_{1}\left(\omega_{1}-\omega_{0}\right)+M_{2}\left(\omega_{2}-\omega_{0}\right)+M_{3}\left(\omega_{3}-\omega_{0}\right)\right] \\
& =\frac{P_{C O I}}{M_{T}}\left[-\omega_{0}\left(M_{1}+M_{2}+M_{3}\right)+\left(M_{1} \omega_{1}+M_{2} \omega_{2}+M_{3} \omega_{3}\right)\right] \\
& \text { substituting from Equation }(3.15) \\
& =0
\end{aligned}
$$

Finally, the resultant equation is found in the form of Equation (3.27). 


\section{Appendix B}

\section{Energy Function for a Lossless}

\section{SMIB System and the Equal Area \\ Criterion (EAC)}

This section interprets an energy function derived for an SMIB system using the EAC. The motion of the system, represented in Equation (3.24) connected to an infinite bus, is shown in Equation (B.1). $\frac{M_{i}}{M_{T}} P_{C O I} \approx 0(i \neq m)$ due to the very large inertia constant of the infinite bus, $M_{m}$. Further, $\delta_{m}=0$ enables the COI variables

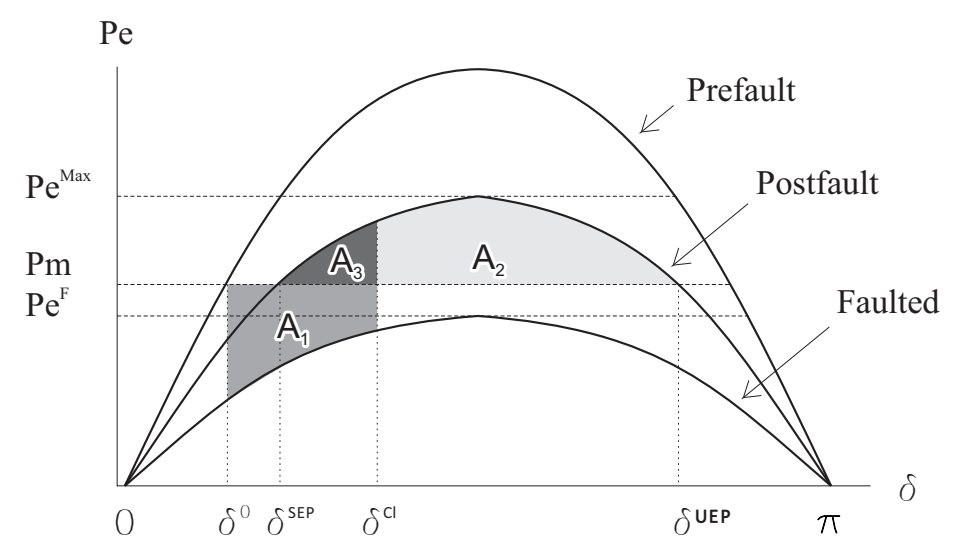

Figure B-1: SMIB system representation using EAC 
$\theta_{i}=\delta_{i}-\delta_{m}$ and $\tilde{\omega}_{i}=\omega_{i}-\omega_{m}$ become $\theta_{i}=\delta_{i}$ and $\tilde{\omega}_{i}=\omega_{i}$

$$
M \frac{d^{2} \delta}{d t^{2}}=P_{m}-P_{e}^{\max } \sin \delta
$$

Multiplying the Equation (B.1) by $\frac{d \theta}{d t}$ leads to

$$
M \frac{d}{d t}\left[\frac{d \delta}{d t^{2}}\right]^{2}=\frac{d \theta}{d t}\left[\frac{-\partial V_{P E}(\delta)}{\partial \delta}\right]
$$

where $V_{P E}(\delta)=-P_{m} \delta-P_{e}^{\max } \cos \delta$

$$
\frac{d}{d t} \omega^{2}=2 \omega \frac{d \omega}{d t}=2 \frac{d}{d t}\left(\frac{d \delta}{d t}\right)^{2}
$$

therefore,

$$
\frac{d}{d t}\left(\frac{d \delta}{d t}\right)^{2}=\frac{1}{2} \frac{d}{d t} \omega^{2}
$$

hence,

$$
\frac{d}{d t}\left[\frac{1}{2} M \omega^{2}+V_{P E}(\delta)\right]=0
$$

The energy function $V(\delta, \omega)=\frac{1}{2} M \omega^{2}+V_{P E}(\delta)$ is a valid energy function. At equilibrium points, from Equation (B.1), $\delta=\sin ^{-1}\left(\frac{P_{m}}{P_{m a x}^{e}}\right)$. To make $V_{P E}=0$ at the SEP, the equation is rearranged as Equation (B.6)

$$
V(\delta, \omega)=\frac{1}{2} M \omega^{2}-P_{m}\left(\delta-\delta^{s}\right)-P_{e}^{\max }\left(\cos \delta-\cos \delta^{s}\right)
$$

Variation of potential energy with respect to the rotor angle variation of an SMIB system obtained from a Matlab simulation is shown in Figure 3-8. Potential energy reaches a maxima at UEPs. The SMIB network is modelled according to the data given in the example 13.1 of [7]. 


\section{Interpretation Using the EAC}

In Figure B-1, $\delta^{0}$ is the pre-fault SEP, $\delta^{S E P}$ is the post-fault SEP, $\delta^{c l}$ is the rotor angle at the fault clearing time, $\delta^{U E P}$ is the UEP. In terms of the EAC, the system is stable if the areas $A_{1}<A_{2}$.

The area $A_{1}$ is given by $\int_{\delta^{0}}^{\delta^{c l}}\left(P_{m}-P_{e}^{F} \sin \delta\right) d \delta$. Further simplification leads the expression $A_{1}=\frac{1}{2} M\left(\omega^{c l}\right)^{2}$. Therefore, $A_{1}$ is the kinetic energy injected to the system during the faulted period.

The area $A_{2}$ is given by $\int_{\delta^{c l}}^{\delta^{U E P}}\left(P_{e}^{M a x} \sin \delta-P_{m}\right) d \delta=-P_{e}^{M a x}\left(\cos \delta^{U E P}-\cos \delta^{c l}\right)-$ $P_{m}\left(\delta^{U E P}-\delta^{c l}\right)$. Further simplification leads to the expression $A_{2}=V_{P E}\left(\delta^{U E P}\right)-$ $V_{P E}\left(\delta^{c l}\right)$.

By adding $A_{3}$ to both sides of the assessment criterion $A_{1}<A_{2}$, the new criteria $A_{1}+A_{3}<A_{2}+A_{3}$ is obtained.

The area $A_{3}$ is given by $\int_{\delta^{S E P}}^{\delta^{c l}}\left(P_{e}^{\max } \sin \delta-P_{m}\right) d \delta$. Further simplification leads to $A_{3}=-P_{m}\left(\delta^{c l}-\delta^{S E P}\right)-P_{e}^{M a x}\left(\cos \delta^{c l}-\cos \delta^{s}\right)$.

The rotor angle increase due to acceleration results the potential energy maximum at the UEP at $\delta=\pi-\delta^{S E P}$. From Equation (B.6), the potential energy at the UEP is obtained as $\left.V(\delta, \omega)\right|_{\delta=\delta^{U E P}, \omega=0}=-P_{m}\left(\pi-2 \delta^{s}\right)+2 P-e^{\max } \cos \delta^{s}=A_{2}+A_{3}$ which is denoted by $V_{c r}$.

The area $A 1+A 3$ is given by Equation (B.6) for any $\delta$ and $\omega$ which is the energy acquired at the fault clearing time. Therefore, the stability assessment criterion $A 1<A 2$ of EAC is equivalent to the direct method assessment criterion $V(\delta, \omega)<V_{c r}$ [48]. 


\section{Appendix C}

\section{Test System Data: IEEE 10 Generator 39 Bus System}

Table C.1: Real and reactive power at load buses of the IEEE 10 generator 39 bus power system

\begin{tabular}{crr}
\hline \hline Bus Number & $P_{\text {load }}(M W)$ & $Q_{\text {load }}($ MVar $)$ \\
\hline 3 & 322 & 2.4 \\
4 & 500 & 184 \\
7 & 233.8 & 84 \\
8 & 522 & 176 \\
12 & 7.5 & 88 \\
15 & 320 & 153 \\
16 & 329 & 32.3 \\
18 & 158 & 30 \\
20 & 628 & 103 \\
21 & 274 & 115 \\
23 & 247.5 & 84.6 \\
24 & 308.6 & -92.2 \\
25 & 224 & 47.2
\end{tabular}


Table C.1: Real and reactive power at load buses of the IEEE 10 generator 39 bus power system

\begin{tabular}{ccr}
\hline \hline Bus Number & $P_{\text {load }}(M W)$ & $Q_{\text {load }}($ MVar $)$ \\
\hline 26 & 139 & 17 \\
27 & 281 & 75.5 \\
28 & 206 & 27.6 \\
29 & 283.5 & 26.9 \\
31 & 9.2 & 4.6 \\
39 & 1104 & 250 \\
\hline \hline
\end{tabular}

Table C.2: Transmission Line Data of the IEEE 10 generator 39 bus power system. The quantities are specified in per unit on 100MVA

\begin{tabular}{ccrrr}
\hline \hline To bus & From bus & Resistance & Reactance & Susceptance \\
\hline 1 & 2 & 0.0035 & 0.0411 & 0.6987 \\
1 & 39 & 0.001 & 0.025 & 0.75 \\
2 & 3 & 0.0013 & 0.0151 & 0.2572 \\
2 & 25 & 0.007 & 0.0086 & 0.146 \\
3 & 4 & 0.0013 & 0.0213 & 0.2214 \\
3 & 18 & 0.0011 & 0.0133 & 0.2138 \\
4 & 5 & 0.0008 & 0.0128 & 0.1342 \\
4 & 14 & 0.0008 & 0.0129 & 0.1382 \\
5 & 6 & 0.0002 & 0.0026 & 0.0434 \\
5 & 8 & 0.0008 & 0.0112 & 0.1476 \\
6 & 7 & 0.0006 & 0.0092 & 0.113 \\
6 & 11 & 0.0007 & 0.0082 & 0.1389 \\
7 & 8 & 0.0004 & 0.0046 & 0.078 \\
8 & 9 & 0.0023 & 0.0363 & 0.3804
\end{tabular}


Table C.2: Transmission Line Data of the IEEE 10 generator 39 bus power system. The quantities are specified in per unit on 100MVA

\begin{tabular}{|c|c|c|c|c|}
\hline To bus & From bus & Resistance & Reactance & Susceptance \\
\hline 9 & 39 & 0.001 & 0.025 & 1.2 \\
\hline 10 & 11 & 0.0004 & 0.0043 & 0.0729 \\
\hline 10 & 13 & 0.0004 & 0.0043 & 0.0729 \\
\hline 13 & 14 & 0.0009 & 0.0101 & 0.1723 \\
\hline 14 & 15 & 0.0018 & 0.0217 & 0.366 \\
\hline 15 & 16 & 0.0009 & 0.0094 & 0.171 \\
\hline 16 & 17 & 0.0007 & 0.0089 & 0.1342 \\
\hline 16 & 19 & 0.0016 & 0.0195 & 0.304 \\
\hline 16 & 21 & 0.0008 & 0.0135 & 0.2548 \\
\hline 16 & 24 & 0.0003 & 0.0059 & 0.068 \\
\hline 17 & 18 & 0.0007 & 0.0082 & 0.1319 \\
\hline 17 & 27 & 0.0013 & 0.0173 & 0.3216 \\
\hline 21 & 22 & 0.0008 & 0.014 & 0.2565 \\
\hline 22 & 23 & 0.0006 & 0.0096 & 0.1846 \\
\hline 23 & 24 & 0.0022 & 0.035 & 0.361 \\
\hline 25 & 26 & 0.0032 & 0.0323 & 0.513 \\
\hline 26 & 27 & 0.0014 & 0.0147 & 0.2396 \\
\hline 26 & 28 & 0.0043 & 0.0474 & 0.7802 \\
\hline 26 & 29 & 0.0057 & 0.0625 & 1.029 \\
\hline 28 & 29 & 0.0014 & 0.0151 & 0.249 \\
\hline
\end{tabular}


Table C.3: Generator dynamic parameters - Salient Pole Generator Model

\begin{tabular}{crrrrrrrrrrrrr}
\hline \hline Bus \# & $T_{d o}^{\prime}$ & $T_{d o}^{\prime \prime}$ & $T_{q o}^{\prime \prime}$ & $H$ & $D$ & $X_{d}$ & $X_{q}$ & $X_{d}^{\prime}$ & $X_{d}^{\prime \prime}$ & $X_{l}$ & $S(1.0)$ & $S(1.2)$ & MVA Base \\
\hline 30 & 10.2 & 0.05 & 0.06 & 4.2 & 0.4 & 1 & 0.69 & 0.31 & 0.3 & 0.125 & 0.15 & 0.425 & 1000 \\
31 & 6.56 & 0.05 & 0.06 & 3.03 & 0.975 & 2.95 & 2.82 & 0.697 & 0.6 & 0.35 & 0.07 & 0.391 & 1000 \\
32 & 5.7 & 0.05 & 0.06 & 3.58 & 1 & 2.495 & 2.37 & 0.531 & 0.5 & 0.304 & 0.08 & 0.283 & 1000 \\
33 & 5.69 & 0.05 & 0.06 & 2.86 & 1 & 2.62 & 2.58 & 0.436 & 0.4 & 0.295 & 0.14 & 0.591 & 1000 \\
34 & 5.4 & 0.05 & 0.06 & 2.6 & 0.3 & 6.7 & 6.2 & 1.32 & 1.3 & 0.54 & 0.15 & 0.6 & 1000 \\
35 & 7.3 & 0.05 & 0.06 & 3.48 & 1 & 2.54 & 2.41 & 0.5 & 0.4 & 0.224 & 0.09 & 0.291 & 1000 \\
36 & 5.66 & 0.05 & 0.06 & 2.64 & 0.8 & 2.95 & 2.92 & 0.49 & 0.4 & 0.322 & 0.14 & 0.529 & 1000 \\
37 & 6.7 & 0.05 & 0.06 & 2.43 & 0.9 & 2.9 & 2.8 & 0.57 & 0.5 & 0.28 & 0.08 & 0.268 & 1000 \\
38 & 4.79 & 0.05 & 0.06 & 3.45 & 1.4 & 2.106 & 2.05 & 0.57 & 0.5 & 0.298 & 0.11 & 0.447 & 1000 \\
39 & 7 & 0.05 & 0.06 & 50 & 1 & 0.2 & 0.19 & 0.06 & 0.05 & 0.03 & 0 & 0 & 1200 \\
\hline \hline
\end{tabular}


Table C.4: Exciter parameters - IEEE Type 1 Excitation System

\begin{tabular}{|c|c|c|c|c|c|c|c|c|c|c|c|c|c|c|}
\hline Bus \# & $\mathrm{TR}$ & $\mathrm{KA}$ & $\mathrm{TA}$ & VRMAX & VRMIN & $\mathrm{KE}$ & $\mathrm{TE}(>0)$ & $\mathrm{KF}$ & $\mathrm{TF}(>0)$ & Switch & E1 & $\mathrm{SE}(\mathrm{E} 1)$ & $\mathrm{E} 2$ & $\mathrm{SE}(\mathrm{E} 2)$ \\
\hline 30 & 0.001 & 5 & 0.06 & 10 & -10 & 1 & 0.25 & 0.04 & 1 & 0 & 3.55 & 0.08 & 4.73 & 0.26 \\
\hline 31 & 0.001 & 6.2 & 0.05 & 10 & -10 & 0.06 & 0.405 & 0.057 & 0.5 & 0 & 3.03 & 0.66 & 4.05 & 0.88 \\
\hline 32 & 0.001 & 5 & 0.06 & 10 & -10 & 1 & 0.5 & 0.08 & 1 & 0 & 2.34 & 0.13 & 3.12 & 0.34 \\
\hline 33 & 0.001 & 5 & 0.06 & 10 & -10 & 1 & 0.5 & 0.08 & 1 & 0 & 2.87 & 0.08 & 3.82 & 0.314 \\
\hline 34 & 0.001 & 20 & 0.02 & 10 & -10 & 1 & 0.785 & 0.03 & 1 & 0 & 3.93 & 0.07 & 5.24 & 0.91 \\
\hline 35 & 0.001 & 5 & 0.02 & 10 & -10 & 1 & 0.471 & 0.0754 & 1.246 & 0 & 3.59 & 0.064 & 4.78 & 0.251 \\
\hline 36 & 0.001 & 40 & 0.02 & 10 & -10 & 1 & 0.73 & 0.03 & 1 & 0 & 2.8 & 0.53 & 3.74 & 0.74 \\
\hline 37 & 0.001 & 5 & 0.02 & 10 & -10 & 1 & 0.528 & 0.0854 & 1.26 & 0 & 3.19 & 0.072 & 4.26 & 0.282 \\
\hline 38 & 0.001 & 40 & 0.02 & 10.5 & -10 & 1 & 1.4 & 0.03 & 1 & 0 & 4.26 & 0.62 & 5.68 & 0.85 \\
\hline 39 & 0.001 & 40 & 0.02 & 10.5 & -10 & 1 & 1.4 & 0.03 & 1 & 0 & 4.26 & 0.62 & 5.68 & 0.85 \\
\hline
\end{tabular}


Table C.5: PSS parameters - Speed sensitive stabilizing model (STAB1)

\begin{tabular}{rrrrrrrr}
\hline \hline Bus \# & $\mathrm{K} / \mathrm{T}$ & $\mathrm{T}$ & $\mathrm{T} 1 / \mathrm{T} 3$ & $\mathrm{~T} 3$ & $\mathrm{~T} 2 / \mathrm{T} 4$ & $\mathrm{~T} 4$ & HLIM \\
\hline 39 & 38 & 0.05 & 5 & 0.09 & 5 & 0.09 & 10 \\
38 & 15 & 0.05 & 4.5 & 0.07 & 4.5 & 0.07 & 10 \\
37 & 2.5 & 0.05 & 7.8 & 0.04 & 7.8 & 0.04 & 10 \\
35 & 8 & 0.05 & 4.2 & 0.05 & 4.2 & 0.05 & 10 \\
34 & 15 & 0.05 & 3.5 & 0.07 & 3.5 & 0.07 & 10 \\
33 & 2.5 & 0.05 & 3.7 & 0.1 & 3.7 & 0.1 & 10 \\
32 & 17 & 0.05 & 5.8 & 0.04 & 5.8 & 0.04 & 10 \\
31 & 6 & 0.05 & 5.5 & 0.07 & 5.5 & 0.07 & 10 \\
30 & 14 & 0.05 & 6.1 & 0.04 & 6.1 & 0.04 & 10 \\
\hline \hline
\end{tabular}




\section{Appendix D}

\section{Classification using SVM}

\section{D.1 Introduction}

This section presents the SVM problem formulation and the application in brief. This optimization problem is initially formulated for the hard margin SVM allowing no margin violations. The application for linearly non-separable datasets is achieved by the use of Kernel method to map the dataset in the feature space to a higher dimension. Then, the soft margin SVM is introduced to account for the margin violations in the transformed feature space.

\section{D.2 Support Vector Machines}

Separating hyperplanes form linear decision boundaries which explicitly separate data into different classes as much as possible. A linearly separable set of data as shown in Figure D-1 can be separated using any machine learning technique. There can be many solutions for the said classification problem. The new addition in the SVM is to select the best hyperplane for classification. That provides the basis for support vector classifiers. In this context, the best selection is the hyperplane with the largest margin. In the presence of noise, the chances are higher for misclassification for the 


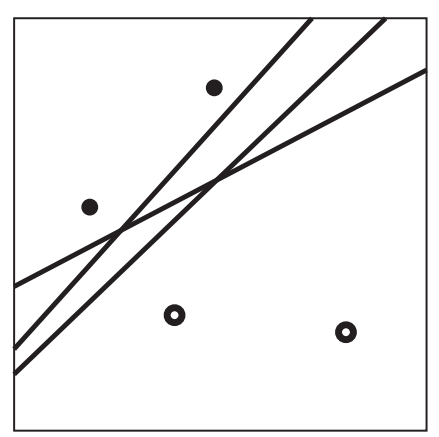

Figure D-1: Possible solutions from different machine learning techniques [81]
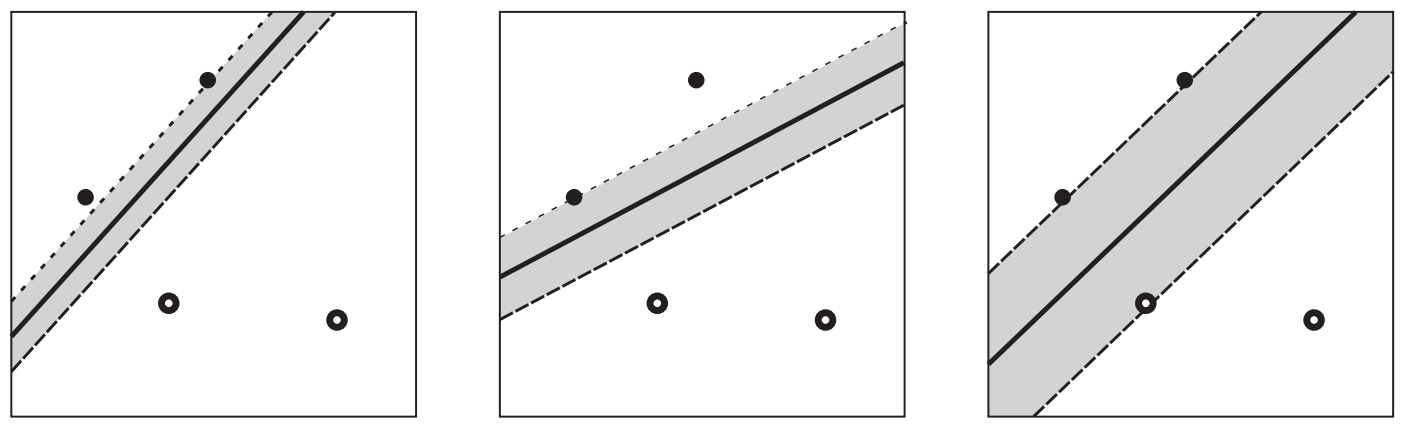

Figure D-2: Margins of different solution shown in Figure D-1

hyperplanes with smaller margins. Figure D-2 shows the available margin at each solution. The hyperplane with the largest margin maximizes the distance to the closest point from either class and is called the optimal separating hyperplane.

\section{D.3 Hard Margin SVM}

The computation of optimally separating hyperplanes leads to the following formulation of the problem for the hard margin SVM. The following formulation is mathematically valid only if there is a feasible linearly separable solution. Once the SVMs are computed the solution can be checked for the existence of misclassification. If misclassifications exist, practically there should be no solution. 


$$
\mathcal{L}(\alpha)=\sum_{n=1}^{N} \alpha_{n}-\frac{1}{2} \sum_{n=1}^{N} \sum_{m=1}^{N} y_{n} y_{m} \alpha_{n} \alpha_{m} x_{n}^{T} x_{m}
$$

Maximize w.r.t. $\alpha$ subject to $\alpha_{n} \geq 0$ for $n=1, \ldots, N$ and $\sum_{n=1}^{N} \alpha_{n} y_{n}=0$

where $\alpha$ is a Lagrangian multiplier. $N$ is the dataset size. $y_{n}$ is the output label, $y_{n} \in-1,+1$, of the data point $x_{n}$. $\alpha$ s having positive values correspond to the support vectors. The other internal points will have zero for respective values of $\alpha$.

\section{D.3.1 Non-linear Transformation to Higher Dimensions}

The above Equation (D.1) is presented assuming the data set is linearly separable. This methodology can be applied to a non-linearly-separable dataset in the same manner by transforming the dataset from $\mathcal{X}$ to a higher dimensional $\mathcal{Z}$ space where the dataset becomes linearly separable. The said transformation changes the form of Equation (D.1) to the following form Equation (D.2).

$$
\begin{aligned}
& \mathcal{L}(\alpha)=\sum_{n=1}^{N} \alpha_{n}-\frac{1}{2} \sum_{n=1}^{N} \sum_{m=1}^{N} y_{n} y_{m} \alpha_{n} \alpha_{m} \boldsymbol{z}_{\boldsymbol{n}}^{\boldsymbol{T}} \boldsymbol{z}_{\boldsymbol{m}} \\
& \text { Constrainits: } \alpha_{n} \geq 0 \text { for } n=1, \ldots, N \text { and } \sum_{n=1}^{N} \alpha_{n} y_{n}=0
\end{aligned}
$$

Equation (D.2) shows that the dimensionality of the $\mathcal{Z}$ domain does not have an impact on the complexity of the solution once the inner product $z^{T} z_{n}$ is calculated. In this case the support vectors are in $\mathcal{Z}$ space. Support vectors in the feature space $(\mathcal{X})$ would look like the highlighted data points in Figure D-3. The distance between the data points and the margins are not the margins of support vectors. The margins are maintained in the $\mathcal{Z}$ space. 


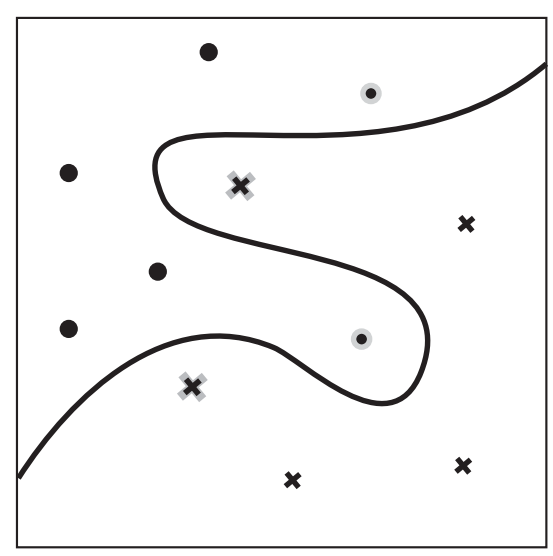

Figure D-3: Suppport vectors in the feature space [81]

\section{D.3.2 Use of Kernels}

The kernels are used to transform a dataset to a very high dimensional $\mathcal{Z}$ space without making the computation process complex. According to Equation (D.2), from the $\mathcal{Z}$ space, only the inner product of $z_{n}^{T} z_{m}$ is required for both $a$ ) the Lagrangian equation, and $b$ ) the inequalities. The kernel methods are capable of providing the inner product of the vectors (in the very high dimensional $\mathcal{Z}$ space) without explicitly transforming the dataset to the $\mathcal{Z}$ space.

\section{D.3.3 RBF Kernel}

Kernel methods achieve flexibility by fitting simple models in a region local to the

target point $x$. Localization is achieved via a weighting kernel. RBF combine these ideas, by treating the kernel functions $K\left(x, x^{\prime}\right)$ as basis functions leads to the model in Equation (D.3).

$$
K\left(x, x^{\prime}\right)=\exp \left(-\gamma\left\|x-x^{\prime}\right\|^{2}\right)
$$

The following example illustrates that the kernel function Equation (D.3) corresponds to the inner product in the infinite dimensional $\mathcal{L}$ space. Assuming both $x$ 
and $x^{\prime}$ are scalars and $\gamma=1$, the expansion of (Equation (D.3)) using the Taylor series, $e^{x}=\sum_{n=0}^{\infty} \frac{x^{n}}{n !}$, leads to Equation (D.4).

$$
\begin{aligned}
K\left(x, x^{\prime}\right) & =\exp \left(-\left\|x-x^{\prime}\right\|^{2}\right) \\
& =\exp \left(-x^{2}\right) \exp \left(-x^{\prime^{2}}\right) \underbrace{\sum_{k=0}^{\infty} \frac{2^{k}(x)^{k}\left(x^{\prime}\right)^{k}}{k !}}_{\exp \left(2 x x^{\prime}\right)}
\end{aligned}
$$

Equation (D.4) can be arranged as the inner product of two vectors, $x$ and $x^{\prime}$ in infinite-dimensional space as the summation continues from zero to infinity.

\section{D.4 Soft Margin SVM}

This section introduces the extension of the linearly separable case to the non-linearly separable case of SVM allowing misclassifications upto a certain extent in order to avoid outliers which results in unduly complex non-linear transformations.

\section{D.4.1 Accounting for Margin Violations}

As shown in Figure D-4, margin violations can be quantified by introducing a slack in potentially every point. Accordingly, Equation (D.1) is modified to Equation (D.5). 


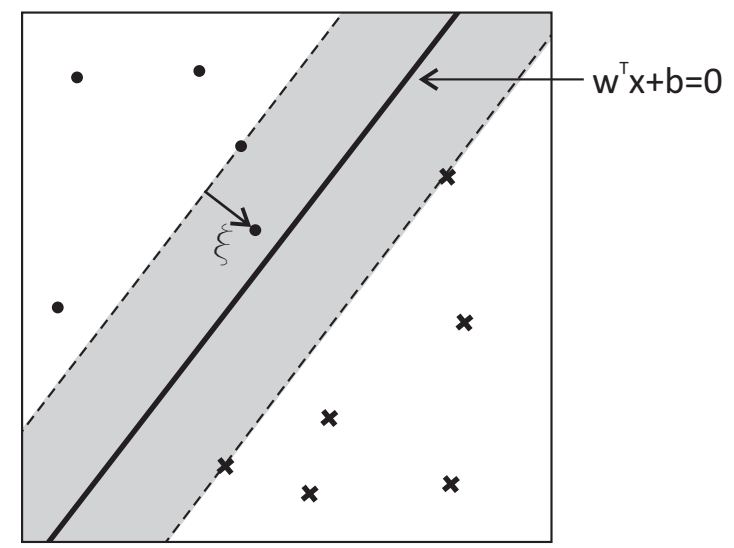

Figure D-4: Margin violation in a linearly separable dataset [81]

The terms introduced to the Lagrange equation to account for margin violations cancel out during simplification resulting the same Lagrange equation as in Equation (D.1). The new addition is the upper limit of $\alpha$ which is the cost parameter, $C$.

$$
\mathcal{L}(\alpha)=\sum_{n=1}^{N} \alpha_{n}-\frac{1}{2} \sum_{n=1}^{N} \sum_{m=1}^{N} y_{n} y_{m} \alpha_{n} \alpha_{m} x_{n}^{T} x_{m}
$$

Maximize w.r.t. $\alpha$

subject to $0 \leq \alpha_{n} \leq C$ for $n=1, \ldots, N$ and $\sum_{n=1}^{N} \alpha_{n} y_{n}=0$

This leads to the introduction of two types of support vectors depending on the value of $\alpha$.

1. Margin support vectors $(0<\alpha<C)$

As $\alpha<C, \beta>0$. When the Lagrange multiplier is greater than zero, the respective slack becomes zero $(\xi=0)$

2. Non margin support vectors $\left(\alpha_{n}=C\right)$

When $\alpha_{n}=C, \beta=0$. When Lagrange multiplier is zero,the respective slack 
becomes positive $(\xi>0)$

Other points where $\alpha=0$ are the inner points.

The value of the cost parameter, $C$, decides how many violations are permitted for a given width of the margin. In practical problems optimum $C$ is determined using cross validation [82]. $\gamma$ in Equation (D.3) determines the best shape of the Gaussian function which is the other parameter to be determined by the cross validation. 


\section{Bibliography}

[1] J. Dagle, "Postmortem analysis of power grid blackouts - the role of measurement systems," Power and Energy Magazine, IEEE, vol. 4, no. 5, pp. 30-35, 2006.

[2] P. Pourbeik, P. Kundur, and C. Taylor, "The anatomy of a power grid blackout - root causes and dynamics of recent major blackouts," Power and Energy Magazine, IEEE, vol. 4, no. 5, pp. 22-29, 2006.

[3] M. Adibi and N. Martins, "Impact of power system blackouts," in Power Energy Society General Meeting, 2015 IEEE, July 2015, pp. 1-15.

[4] L. Wang, "Techniques for high performance analysis of transient stability," in Power and Energy Society General Meeting, 2012 IEEE, 2012, pp. 1-6.

[5] I. Dudurych, A. Rogers, R. Aherne, L. Wang, F. Howell, and X. Lin, "Safety in numbers: Online security analysis of power grids with high wind pentration," Power and Energy Magazine, IEEE, vol. 10, no. 2, pp. 62-70, 2012.

[6] P. Kundur, J. Paserba, V. Ajjarapu, G. Andersson, A. Bose, C. Canizares, N. Hatziargyriou, D. Hill, A. Stankovic, C. Taylor, T. Van Cutsem, and V. Vittal, "Definition and classification of power system stability ieee/cigre joint task force on stability terms and definitions," Power Systems, IEEE Transactions on, vol. 19, no. 3, pp. 1387-1401, 2004.

[7] P. Kundur, N. Balu, and M. Lauby, Power system stability and control, ser. The EPRI power system engineering series. McGraw-Hill, 1994. 
[8] M. Pavella, D. Ernst, and D. Ruiz-Vega, Transient Stability of Power Systems: A Unified Approach to Assessment and Control, ser. Kluwer International Series in Engineering \& Computer Science. Springer London, Limited, 2000.

[9] H. Chiang, Direct Methods for Stability Analysis of Electric Power Systems: Theoretical Foundation, BCU Methodologies, and Applications. John Wiley \& Sons, 2011.

[10] A. M. Lyapunov, The General Problem of the Stability of Motion (in Russian). The Mathematical Society of Kharkov, Russia, 1892. English Translation, Taylor and Francis Ltd., London, 1992.

[11] P. Magnusson, "The transient-energy method of calculating stability," American Institute of Electrical Engineers, Transactions of the, vol. 66, no. 1, pp. 747-755, Jan 1947.

[12] J. Slotine and W. Li, Applied nonlinear control. Prentice Hall, 1991.

[13] H.-D. Chiang, "Study of the existence of energy functions for power systems with losses," Circuits and Systems, IEEE Transactions on, vol. 36, no. 11, pp. 1423-1429, Nov 1989.

[14] N. Kakimoto, Y. Ohnogi, H. Matsuda, and H. Shibuya, "Transient stability analysis of large-scale power system by lyapunov's direct method," Power Apparatus and Systems, IEEE Transactions on, vol. PAS-103, no. 1, pp. 160-167, 1984.

[15] B. Jayasekara and U. D. Annakkage, "Derivation of an accurate polynomial representation of the transient stability boundry," Power Systems, IEEE Transactions on, vol. 21, no. 4, pp. 1856-1863, 2006.

[16] I. Kamwa, S. R. Samantaray, and G. Joos, "Development of rule-based classifiers for rapid stability assessment of wide-area post-disturbance records," Power Systems, IEEE Transactions on, vol. 24, no. 1, pp. 258-270, Feb 2009. 
[17] F. R. Gomez, A. D. Rajapakse, U. D. Annakkage, and I. T. Fernando, "Support vector machine-based algorithm for post-fault transient stability status prediction using synchronized measurements," Power Systems, IEEE Transactions on, vol. 26, no. 3, pp. 1474-1483, 2011.

[18] Y. Xu, Z. Y. Dong, J. H. Zhao, P. Zhang, and K. P. Wong, "A reliable intelligent system for real-time dynamic security assessment of power systems," Power Systems, IEEE Transactions on, vol. 27, no. 3, pp. 1253-1263, 2012.

[19] F. Hashiesh, H. E. Mostafa, A.-R. Khatib, I. Helal, and M. M. Mansour, "An intelligent wide area synchrophasor based system for predicting and mitigating transient instabilities," Smart Grid, IEEE Transactions on, vol. 3, no. 2, pp. 645-652, 2012.

[20] J. Lv, M. Pawlak, and U. Annakkage, "Prediction of the transient stability boundary using the lasso," Power Systems, IEEE Transactions on, vol. 28, no. 1, pp. 281-288, 2013.

[21] M. He, J. Zhang, and V. Vittal, "Robust online dynamic security assessment using adaptive ensemble decision-tree learning," Power Systems, IEEE Transactions on, vol. 28, no. 4, pp. 4089-4098, 2013.

[22] "Transient stability test systems for direct stability methods," Power Systems, IEEE Transactions on, vol. 7, no. 1, pp. 37-43, 1992.

[23] D. You, K. Wang, L. Ye, J. Wu, and R. Huang, "Transient stability assessment of power system using support vector machine with generator combinatorial trajectories inputs," International Journal of Electrical Power $\&$ Energy Systems, vol. 44, no. 1, pp. 318-325, Jan. 2013.

[24] L. Moulin, A. Alves da Silva, M. El-Sharkawi, and I. Marks, R.J., "Support vector machines for transient stability analysis of large-scale power systems," Power Systems, IEEE Transactions on, vol. 19, no. 2, pp. 818-825, 2004. 
[25] M. He, V. Vittal, and J. Zhang, "Online dynamic security assessment with missing pmu measurements: A data mining approach," Power Systems, IEEE Transactions on, vol. 28, no. 2, pp. 1969-1977, 2013.

[26] A. Kaci, I. Kamwa, L. Dessaint, and S. Guillon, "Synchrophasor data baselining and mining for online monitoring of dynamic security limits," Power Systems, IEEE Transactions on, vol. 29, no. 6, pp. 2681-2695, Nov 2014.

[27] H. D. Chiang, Y. Tada, H. Li, and T. Takazawa, "Tepco-bcu for on-line dynamic security assessments of large-scale power systems." Advances in Power System Control, Operation and Management (APSCOM 2009), 8th International Conference on, 2009, pp. 1-14.

[28] Y. Mansour, E. Vaahedi, A. Y. Chang, B. R. Corns, B. W. Garrett, K. Demaree, T. Athay, and K. Cheung, "Bc hydro's on-line transient stability assessment (tsa) model development, analysis and post-processing," vol. 10, no. 1, pp. 241-253, 1995.

[29] E. Vaahedi, Y. Mansour, A. Y. Chang, B. R. Corns, and E. K. Tse, "Enhanced "second kick" methods for on-line dynamic security assessment," vol. 11, no. 4, pp. 1976-1982, 1996.

[30] Y. Mansour, A. Chang, J. Tamby, E. Vaahedi, B. Corns, and M. El-Sharkawi, "Large scale dynamic security screening and ranking using neural networks," Power Systems, IEEE Transactions on, vol. 12, no. 2, pp. 954-960, 1997.

[31] R. Iqbal and S. Krishna, "Online dynamic security assessment of power systems using critical group energy function," in Power India Conference, 2012 IEEE Fifth, 2012, pp. 1-6.

[32] Y. Makarov, P. Du, S. Lu, T. Nguyen, J. Burns, and J. Gronquist, "Wide-area dynamic security region," in North American Power Symposium (NAPS), 2009, 2009, pp. 1-6. 
[33] Y. Makarov, P. Du, S. Lu, T. Nguyen, X. Guo, J. W. Burns, J. Gronquist, and M. A. Pai, "Pmu-based wide-area security assessment: Concept, method, and implementation," Smart Grid, IEEE Transactions on, vol. 3, no. 3, pp. 1325$1332,2012$.

[34] J. Y. Astic, A. Bihain, and M. Jerosolimski, "The mixed adams-bdf variable step size algorithm to simulate transient and long term phenomena in power systems," Power Systems, IEEE Transactions on, vol. 9, no. 2, pp. 929-935, 1994.

[35] J. Sanchez-Gasca, R. D’Aquila, W. Price, and J. Paserba, "Variable time step, implicit integration for extended-term power system dynamic simulation," in Power Industry Computer Application Conference, 1995. Conference Proceedings., 1995 IEEE, 1995, pp. 183-189.

[36] C. A. S. Neto, M. A. Quadros, M. G. Santos, and J. Jardim, "Brazilian system operator online security assessment system," in Power and Energy Society General Meeting, 2010 IEEE, 2010, pp. 1-7.

[37] L. Wang, M. Klein, S. Yirga, and P. Kundur, "Dynamic reduction of large power systems for stability studies," Power Systems, IEEE Transactions on, vol. 12, no. 2, pp. 889-895, 1997.

[38] U. Annakkage, N. K. C. Nair, Y. Liang, A. Gole, V. Dinavahi, B. Gustavsen, T. Noda, H. Ghasemi, A. Monti, M. Matar, R. Iravani, and J. Martinez, "Dynamic system equivalents: A survey of available techniques," Power Delivery, IEEE Transactions on, vol. 27, no. 1, pp. 411-420, Jan 2012.

[39] Y. Xue, T. Van Custem, and M. Ribbens-Pavella, "Extended equal area criterion justifications, generalizations, applications," Power Systems, IEEE Transactions on, vol. 4, no. 1, pp. 44-52, 1989.

[40] User Manual-Transient Security Assessment Tool (TSAT), Powertech Labs Inc., April 2009. 
[41] M. Yin, C. Chung, K. Wong, Y. Xue, and Y. Zou, "An improved iterative method for assessment of multi-swing transient stability limit," Power Systems, IEEE Transactions on, vol. 26, no. 4, pp. 2023-2030, 2011.

[42] H.-D. Chiang, J. Tong, and Y. Tada, "On-line transient stability screening of 14,000-bus models using tepco-bcu: Evaluations and methods," in Proc. IEEE Power and Energy Society General Meeting, 2010, pp. 1-8.

[43] L. Loud, S. Guillon, G. Vanier, J. Huang, L. Riverin, D. Lefebvre, and J. C. Rizzi, "Hydro-quebec's challenges and experiences in on-line dsa applications," in Power and Energy Society General Meeting, 2010 IEEE, 2010, pp. 1-8.

[44] F. Alves, R. Henriques, J. Passos Fo, S. Gomes, C. Borges, D. Falcao, A. Aveleda, G. Taranto, and T. Assis, "Integrating solution engines under a distributed processing environment: An alternative approach for static and dynamic security assessment of large scale power systems," in PES General Meeting - Conference Exposition, 2014 IEEE, July 2014, pp. 1-3.

[45] Z. Huang, R. Diao, S. Jin, and Y. Chen, "Predictive dynamic security assessment through advanced computing," in PES General Meeting - Conference Exposition, 2014 IEEE, July 2014, pp. 1-5.

[46] P. Aristidou and T. Van Cutsem, "Algorithmic and computational advances for fast power system dynamic simulations," in PES General Meeting - Conference Exposition, 2014 IEEE, July 2014, pp. 1-5.

[47] "Load representation for dynamic performance analysis [of power systems]," Power Systems, IEEE Transactions on, vol. 8, no. 2, pp. 472-482, May 1993.

[48] P. Sauer and M. Pai, Power system dynamics and stability. Prentice Hall, 1998. 
[49] P. D. Aylett, "The energy-integral criterion of transient stability limits of power systems," Proceedings of the IEE -Part C: Monographs, vol. 105, no. 8, pp. 527$536,1958$.

[50] A. Michel, A. Fouad, and V. Vittal, "Power system transient stability using individual machine energy functions," Circuits and Systems, IEEE Transactions on, vol. 30, no. 5, pp. 266-276, 1983.

[51] T. Athay, R. Podmore, and S. Virmani, "A practical method for the direct analysis of transient stability," Power Apparatus and Systems, IEEE Transactions on, no. 2, pp. 573-584, 1979.

[52] M. Pai, M. Laufenberg, and P. Sauer, "Some clarifications in the transient energy function method," International Journal of Electrical Power \& Energy Systems, vol. 18 , no. 1, pp. $65-72,1996$.

[53] P. Anderson and A. Fouad, POWER SYSTEM CONTROL AND STABILITY, 2ND ED. Wiley India Pvt. Limited, 2008. [Online]. Available: https: //books.google.ca/books?id=2BXOzA34qBkC

[54] A. A. Fouad and S. E. Stanton, "Transient stability of a multi-machine power system. part ii: Critical transient energy," Power Apparatus and Systems, IEEE Transactions on, vol. PAS-100, no. 7, pp. 3417-3424, 1981.

[55] H. D. Chianag, F. Wu, and P. Varaiya, "Foundations of the potential energy boundary surface method for power system transient stability analysis," Circuits and Systems, IEEE Transactions on, vol. 35, no. 6, pp. 712-728, 1988.

[56] D. P. Wadduwage, J. Geeganage, U. D. Annakkage, and C. Q. Wu, "Investigation of the applicability of lyapunov exponents for transient stability assessment," in Electrical Power Energy Conference (EPEC), 2013 IEEE, Aug 2013, pp. 1-6. 
[57] A. H. El-Abiad and K. Nagappan, "Transient stability regions of multimachine power systems," Power Systems, IEEE Transactions on, no. 2, pp. 169-179, 1966.

[58] H.-D. Chiang, F. Wu, and P. Varaiya, "Foundations of direct methods for power system transient stability analysis," Circuits and Systems, IEEE Transactions on, vol. 34, no. 2, pp. 160-173, 1987.

[59] A. Fouad and V. Vittal, Power System Transient Stability Analysis Using the Transient Energy Function Method. Pearson Education, 1991. [Online]. Available: http://books.google.ca/books?id=rdbVEs07jfYC

[60] A. R. Bergen and D. J. Hill, "A structure preserving model for power system stability analysis," Power Systems, IEEE Transactions on, no. 1, pp. 25-35, 1981.

[61] T. van Cutsem and M. Ribbens-Pavella, "Structure preserving direct methods for transient stability analysis of power systems," in Decision and Control, 1985 24th IEEE Conference on, vol. 24, Dec 1985, pp. 70-76.

[62] N. Tsolas, A. Arapostathis, and P. Varaiya, "A structure preserving energy function for power system transient stability analysis," vol. 32, no. 10, pp. 1041-1049, 1985.

[63] M. Di Pierro, "web2py for scientific applications," Computing in Science 8 Engineering, vol. 13, no. 2, pp. 64-69, 2011. [Online]. Available: http://ieeexplore.ieee.org/stamp/stamp.jsp?arnumber $=5518770$

[64] PSSE Application Program Interface (API), Siemens Energy, Inc., June 2009.

[65] S. Weerasooriya, "Application of neural networks for classification and identification in power systems," Ph.D. dissertation, Univ. Washington, 1992. 
[66] M. D. Pierro, web2py Complete Reference Manual, 5th ed., accessed on August 14, 2013. [Online]. Available: http://www.web2py.com/book

[67] Hwaci. [Online]. Available: http://www.sqlite.org/

[68] T. Hastie, R. Tibshirani, and J. Friedman, The elements of statistical learning: data mining, inference, and prediction, ser. Springer series in statistics. Springer, 2001.

[69] C.-C. Chang and C.-J. Lin, "LIBSVM: A library for support vector machines," ACM Transactions on Intelligent Systems and Technology, vol. 2, pp. 27:1-27:27, 2011, software available at http://www.csie.ntu.edu.tw/ cjlin/libsvm.

[70] M. Pai, Energy Function Analysis for Power System Stability, ser. Kluwer international series in engineering and computer science. Springer, 1989.

[71] V. Azbe, U. Gabrijel, D. Povh, and R. Mihalic, "The energy function of a general multimachine system with a unified power flow controller," Power Systems, IEEE Transactions on, vol. 20, no. 3, pp. 1478-1485, Aug 2005.

[72] R. Mihalic and U. Gabrijel, "A structure-preserving energy function for a static series synchronous compensator," Power Systems, IEEE Transactions on, vol. 19, no. 3, pp. 1501-1507, Aug 2004.

[73] N. Fernandopulle and R. T. H. Alden, "Incorporation of detailed hvdc dynamics into transient energy functions," Power Systems, IEEE Transactions on, vol. 20, no. 2, pp. 1043-1052, May 2005.

[74] RTDS Hardware Manual, RTDS Technologies, January 2009.

[75] J. R. Marti, "Accurate modelling of frequency-dependent transmission lines in electromagnetic transient simulations," IEEE Transactions on Power Apparatus and Systems, vol. PAS-101, no. 1, pp. 147-157, Jan 1982. 
[76] J. Geeganage, U. Annakkage, T. Weekes, and B. Archer, "Application of energybased power system features for dynamic security assessment," Power Systems, IEEE Transactions on, vol. 30, no. 4, pp. 1957-1965, July 2015.

[77] J. Geeganage, U. Annakkage, M. Weekes, and B. Archer, "Application of energybased power system features for dynamic security assessment," in Power Energy Society General Meeting, 2015 IEEE, July 2015, pp. 1-1.

[78] J. Geeganage, U. Annakkage, B. Archer, T. Weekes, and H. Meiklejohn, "Realtime testing of dynamic security assessment algorithms," in CIGRE International Symposium, Lund, Sweden, May 2015.

[79] J. Geeganage and U. D. annakkage, "Estimation of transient energy terms using machine learning," in 2014 CIGRE Canada Conference, 2014.

[80] J. Geeganage, U. Annakkage, B. Archer, and T. Weekes, "A web based software system for database generation for online dynamic security assessment studies (ml4dsa)," in Electrical and Computer Engineering (CCECE), 2013 26th Annual IEEE Canadian Conference on, 2013, pp. 1-4.

[81] Y. Abu-Mostafa, M. Magdon-Ismail, and H. Lin, Learning from Data: A Short Course. AMLBook.com, 2012.

[82] Y. Abu-Mostafa. (2013, August) Learning from data - machine learning course recorded at a live broadcast from caltech. California Institute of Technology. [Online]. Available: http://work.caltech.edu/telecourse.html 